\title{
$\alpha$-Chiral Amines via Thermally Promoted Deaminative Addition of Alkylpyridinium Salts to Sulfinimines
}

Kristen M. Baker, ${ }^{\dagger}$ Amanda Tallon,${ }^{\dagger}$ Richard P. Loach, ${ }^{\dagger}$ Olivia P. Bercher, ${ }^{\dagger}$ Matthew A. Perry, ${ }^{\ddagger}, *$ Mary P.

$$
\begin{gathered}
\text { Watson }^{\dagger, *} \\
{ }^{\dagger} \text { Department of Chemistry \& Biochemistry, University of Delaware, Newark, Delaware, 19716, United States } \\
\text { ₹ Pfizer Medicinal Sciences, Eastern Point Rd., Groton, Connecticut, 06340, United States } \\
{ }^{*} \text { mpwatson@udel.edu, Matthew.Perry@pfizer.com }
\end{gathered}
$$

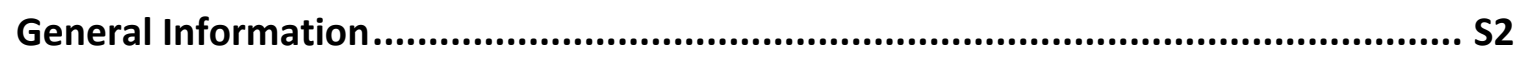

Optimization Studies: Stoichiometric Reductant................................................... S3

Optimization Studies: Photochemical Conditions ................................................ S4

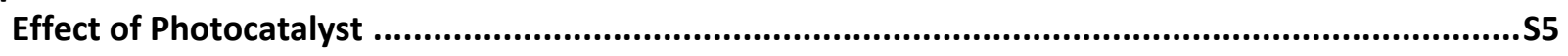

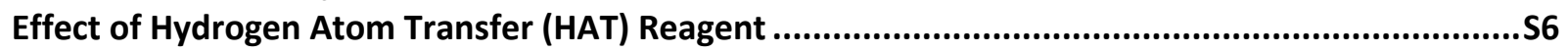

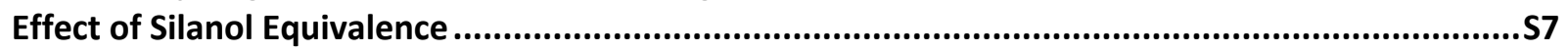

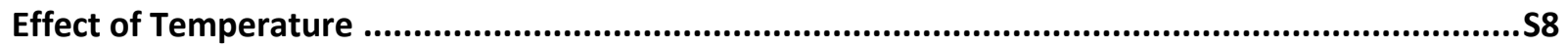

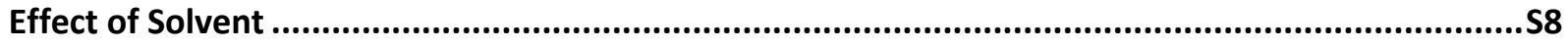

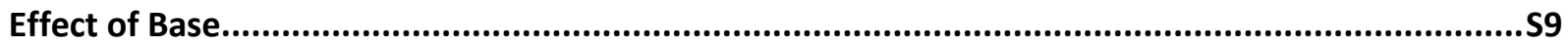

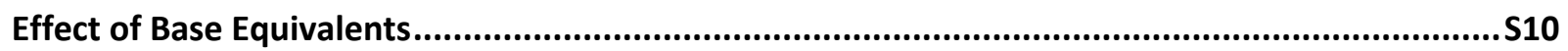

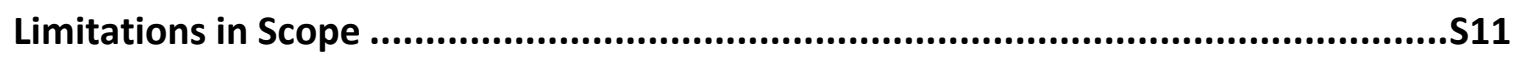

Radical Addition of Alkylpyridinium Salts to Sulfinimines ......................................S13

General Procedure A: Coupling of Alkylpyridinium Salts and Sulfinimines....................................S13

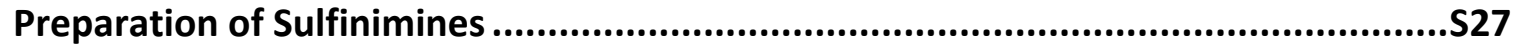

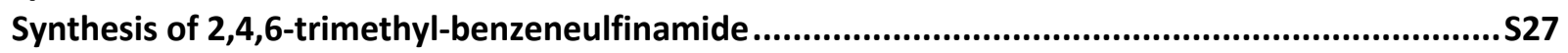

General Procedure B: Synthesis of Sulfinimines ..........................................................................S28

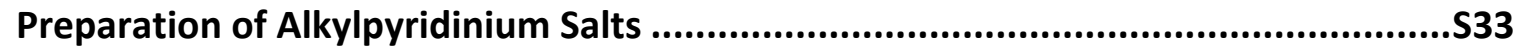

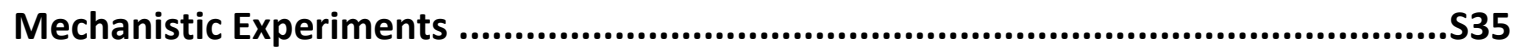

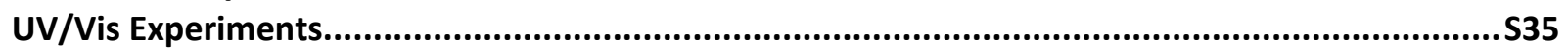

Determination of the Stoichiometry of the Anion $-\pi$ Complex ...................................................S36

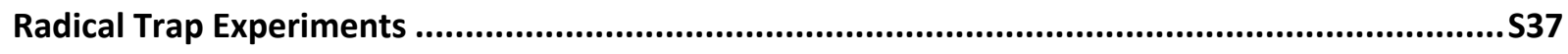

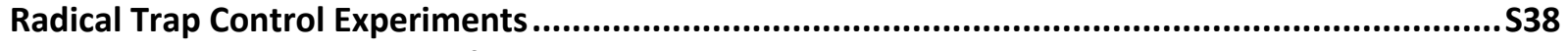

Control Experiments Probing for Pyridinium Ylide Formation ....................................................S39

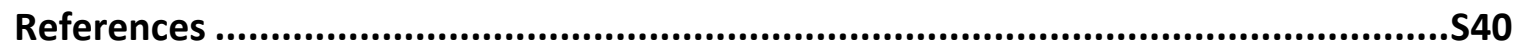

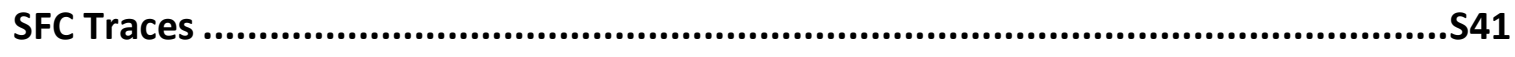

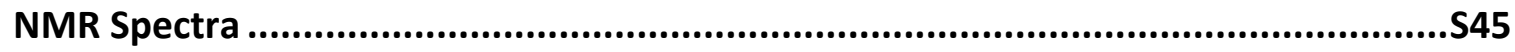




\section{General Information}

Reactions were performed in oven-dried Schlenk flasks or oven-dried round-bottomed flasks unless otherwise noted. Schlenk and round-bottomed flasks were fitted with rubber septa, and reactions were conducted under an atmosphere of $\mathrm{N}_{2}$. Stainless steel syringes or cannulae were used to transfer air- and moisture-sensitive liquids. Flash chromatography was performed on silica gel 60 (40-63 $\mu \mathrm{m}, 60 \AA)$ unless otherwise noted. Commercial reagents, including 2,4,6triphenylpyrylium tetrafluoroborate were purchased from Sigma Aldrich, Acros, AstaTech, Fisher, Strem, TCI, Combi Blocks, Alfa Aesar, AK Scientific, Bide Pharmatech, Oakwood, Ambeed, or Cambridge Isotopes Laboratories and used as received with the following exceptions: $\mathrm{CH}_{2} \mathrm{Cl}_{2}$ was dried by passing through drying columns. ${ }^{1} 4 \AA$ Molecular sieves were purchased and heated under vacuum then crushed and stored in a desiccator. Proton nuclear magnetic resonance $\left({ }^{1} \mathrm{H}\right.$ NMR) spectra, carbon nuclear magnetic resonance $\left({ }^{13} \mathrm{C} \mathrm{NMR}\right)$ spectra, and fluorine nuclear magnetic resonance spectra ( ${ }^{19} \mathrm{~F}$ NMR) were recorded on both $400 \mathrm{MHz}$ and $600 \mathrm{MHz}$ spectrometers. Chemical shifts for protons are reported in parts per million downfield from tetramethylsilane and are referenced to residual protium in the $\mathrm{NMR}$ solvent $\left(\mathrm{CHCl}_{3}=\delta 7.26\right)$. Chemical shifts for carbon are reported in parts per million downfield from tetramethylsilane and are referenced to the carbon resonances of the solvent $\left(\mathrm{CDCl}_{3}=\delta\right.$ 77.16). Chemical shifts for fluorine were externally referenced to $\mathrm{CFCl}_{3}$ in $\mathrm{CDCl}_{3}\left(\mathrm{CFCl}_{3}=\delta 0\right)$. Data are represented as follows: chemical shift, multiplicity $(\mathrm{br}=$ broad, $\mathrm{s}=$ singlet, $\mathrm{d}=$ doublet, $\mathrm{t}=$ triplet, $\mathrm{q}=$ quartet, $\mathrm{p}=$ pentet, $\mathrm{m}=$ multiplet, $\mathrm{dd}=$ doublet of doublets, $\mathrm{ddd}=$ doublet of doublets of doublets, $\mathrm{dt}=$ doublet of triplets, $\mathrm{dq}=$ doublet of quartets, $\mathrm{dp}=$ doublet of pentets, $\mathrm{tt}=$ triplet of triplets, $\mathrm{td}=$ triplet of doublets, $\mathrm{h}=$ heptet), coupling constants in Hertz (Hz), integration. Infrared (IR) spectra were obtained using FTIR spectrophotometers with material loaded onto a $\mathrm{KBr}$ plate. The mass spectral data were obtained at the University of Delaware using a Q-Exactive Orbitrap Mass Spectrometer (Thermo Scientific). Melting points were taken on a Thomas-Hoover Uni-Melt Capillary Melting Point Apparatus. 


\section{Optimization Studies: Stoichiometric Reductant}<smiles>Cc1cc(C)c(S(=O)/N=C/c2cccnc2)c(C)c1</smiles>

General Optimization Procedure. On the benchtop, zinc, alkylpyridinium salt 1a (120.0 mg, $0.25 \mathrm{mmol}, 2.5$ equiv), and sulfinimine $\mathbf{2 a}(27.2 \mathrm{mg}, 0.10 \mathrm{mmol}, 1.0$ equiv) were added to an ovendried 1-dram vial fitted with a stir bar. DMA and the alcohol additive were added via syringe in the ratio shown in the table below. The total volume of solvent was $1.0 \mathrm{~mL}$. The vial was capped with a Teflon-lined cap. The mixture was stirred for $24 \mathrm{~h}$ at $50{ }^{\circ} \mathrm{C}$ in an aluminum heating block. The mixture was then diluted with EtOAc $(3 \mathrm{~mL})$ and filtered through a plug of Celite ${ }^{\circledR}$, which was rinsed with EtOAc $(10 \mathrm{~mL})$. The filtrate was concentrated. 1,3,5-Trimethoxybenzene (internal standard) was added. $\mathrm{CDCl}_{3}$ was added, and the yield was determined by ${ }^{1} \mathrm{H} \mathrm{NMR}$ analysis. Changes to this general procedure are noted in the table below.

Please note: Entries 5-7 were set up identically and represent the lack of reproducibility observed.

\begin{tabular}{ccccc} 
entry & additive & additive:DMA & Zn equiv & ${\text { yield }(\%)^{a}}^{a}$ \\
\hline 1 & ethanol (1.0 equiv) & $1: 167$ & 2 & 17 \\
2 & ethanol & $1: 3$ & 2 & 23 \\
3 & ethylene glycol & $1: 3$ & 2 & 39 \\
4 & ethylene glycol & $1: 1$ & 2 & 51 \\
5 & ethylene glycol & $1: 1$ & 10 & 72 \\
6 & ethylene glycol & $1: 1$ & 10 & 60 \\
7 & ethylene glycol & $1: 1$ & 10 & 47 \\
\hline
\end{tabular}

${ }^{a}$ Determined by ${ }^{1} \mathrm{H}$ NMR analysis using 1,3,5-trimethoxybenzene as internal standard. 


\section{Optimization Studies: Photochemical Conditions}

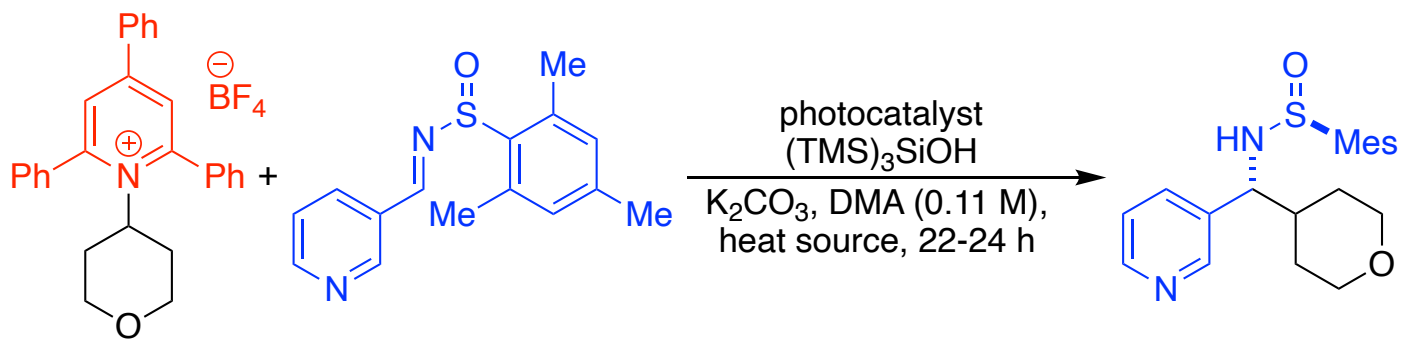

General Optimization Procedure. In a $\mathrm{N}_{2}$-atmosphere glovebox, photocatalyst $(0.0025$ mmol, $2.5 \mathrm{~mol} \%$ ), alkylpyridinium salt $1 \mathrm{a}$ (120.0 mg, $0.25 \mathrm{mmol}, 2.5$ equiv), sulfinimine 2a (27.2 $\mathrm{mg}, 0.10 \mathrm{mmol}, 1.0$ equiv), and $\mathrm{K}_{2} \mathrm{CO}_{3}(69.1 \mathrm{mg}, 0.50 \mathrm{mmol}, 5.0$ equiv) were added to an ovendried 1-dram vial fitted with a stir bar. DMA $(0.9 \mathrm{~mL}, 0.11 \mathrm{M})$ was added via syringe followed by $(\mathrm{TMS})_{3} \mathrm{SiOH}(41 \mu \mathrm{L}, 0.15 \mathrm{mmol}, 1.5$ equiv). The vial was capped with a Teflon-lined cap and removed from the glovebox. The mixture was stirred for $24 \mathrm{~h}$, unless otherwise stated. If heated, an aluminum heating block was used. The mixture was then diluted with EtOAc ( $3 \mathrm{~mL})$ and filtered through a plug of Celite ${ }^{\circledR}$, which was rinsed with EtOAc $(10 \mathrm{~mL})$. The filtrate was concentrated. 1,3,5-Trimethoxybenzene (internal standard) was added. $\mathrm{CDCl}_{3}$ was added, and the yield was determined by ${ }^{1} \mathrm{H}$ NMR analysis. Changes to this general procedure are noted in the tables below. 


\section{Effect of Photocatalyst}

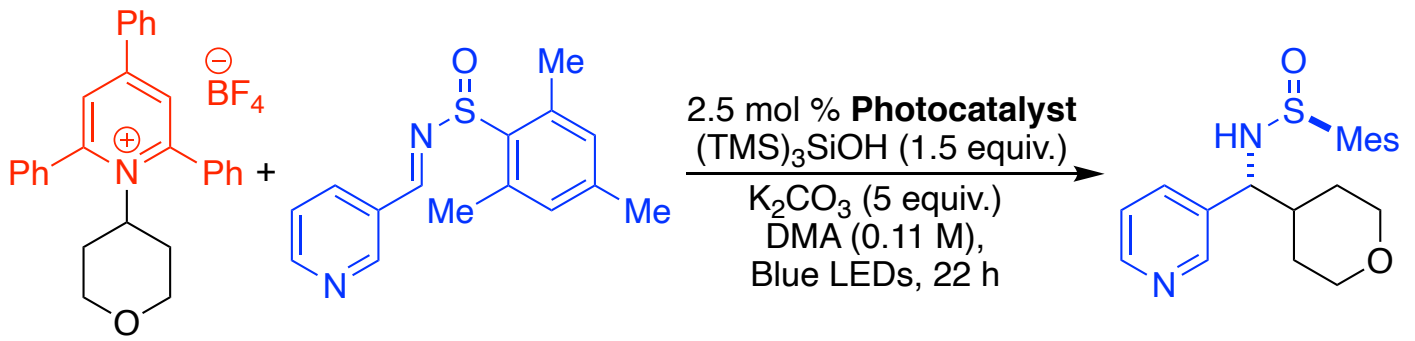

\begin{tabular}{ccc} 
entry & photocatalyst & yield $(\%)^{a}$ \\
\hline 1 & $\operatorname{Ir}((\mathrm{dtbbpy}) \text { ppy })_{2} \mathrm{PF}_{6}$ & 23 \\
2 & $\mathrm{Ir}\left(\mathrm{dF}\left(\mathrm{CF}_{3}\right) \text { ppy }\right)_{2}\left(\mathrm{dtbpy}_{\mathrm{P}} \mathrm{PF}_{6}\right.$ & 21 \\
3 & $\mathrm{Ru}(\mathrm{dmbpy})_{3} \mathrm{PF}_{6}$ & 14 \\
4 & $4 \mathrm{CzIPN}$ & 24 \\
5 & $2 \mathrm{CzIPN}$ & 39 \\
6 & $5 \mathrm{CzBN}$ & 48 \\
7 & None & 48 \\
\hline
\end{tabular}

a Determined by ${ }^{1} \mathrm{H}$ NMR analysis using 1,3,5-trimethoxybenzene as internal standard. 


\section{Effect of Hydrogen Atom Transfer (HAT) Reagent}

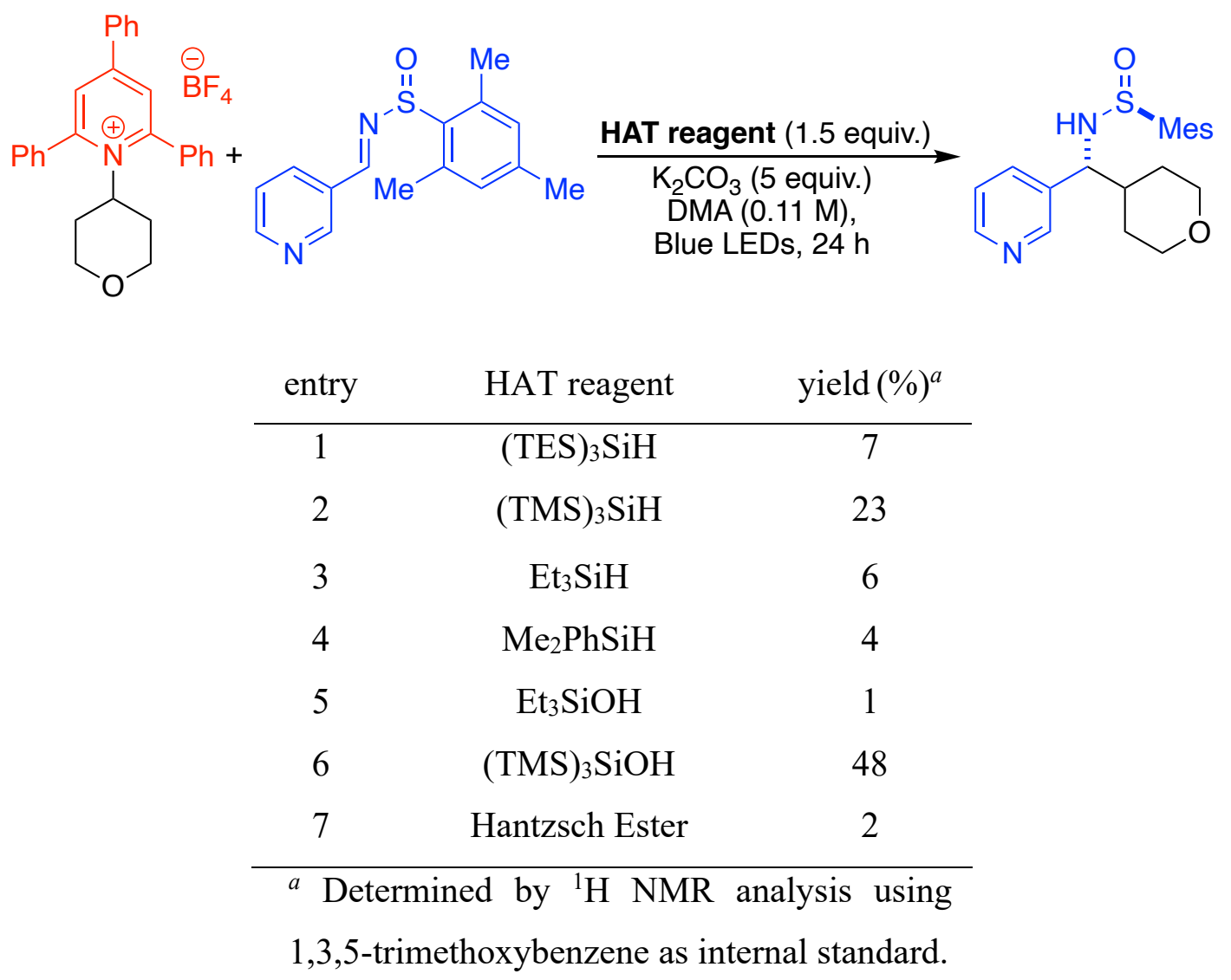




\section{Effect of Silanol Equivalence}

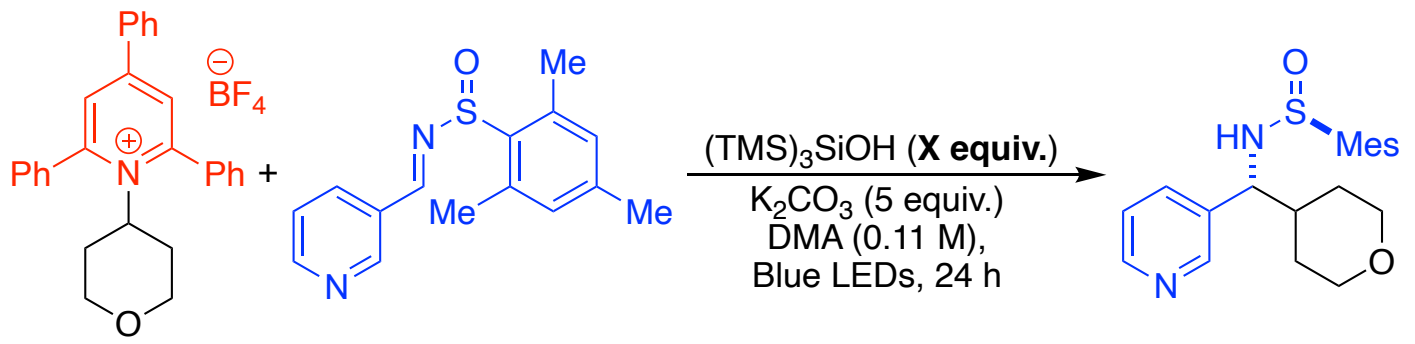

\begin{tabular}{ccc} 
entry & silanol equiv. & yield $(\%)^{a}$ \\
\hline 1 & 0.5 & 14 \\
2 & 1.0 & 27 \\
3 & 1.3 & 27 \\
4 & 1.5 & 48 \\
5 & 2.0 & 39 \\
6 & 2.5 & 39 \\
7 & 3.0 & 49 \\
\hline$a$ & Determined by ${ }^{1} \mathrm{H}$ NMR analysis using \\
$1,3,5$-trimethoxybenzene as internal standard.
\end{tabular}


Effect of Temperature<smiles>Cc1cc(C)c(S(=O)/N=C/c2cccnc2)c(C)c1</smiles>

\begin{tabular}{ccc} 
entry & temperature $\left({ }^{\circ} \mathrm{C}\right)$ & yield $(\%)^{a}$ \\
\hline 1 & 28 & 6 \\
2 & 40 & 45 \\
3 & 50 & 52 \\
4 & 60 & 38 \\
5 & 70 & 32 \\
6 & 80 & 27 \\
\hline
\end{tabular}

${ }^{a}$ Determined by ${ }^{1} \mathrm{H}$ NMR analysis using

1,3,5-trimethoxybenzene as internal standard.

\section{Effect of Solvent}<smiles>c1ccc(-c2cc(-c3ccccc3)c(-c3ccccc3)c(-c3ccccc3)c2)cc1</smiles><smiles>Cc1cc(C)c(S(=O)/N=C/c2cccnc2)c(C)c1</smiles>

\begin{tabular}{ccc} 
entry & solvent & yield $(\%)^{a}$ \\
\hline 1 & DMA & 52 \\
2 & DMF & 51 \\
3 & NMP & 37 \\
4 & DCE & 3 \\
5 & DCM & 10
\end{tabular}

${ }^{a}$ Determined by ${ }^{1} \mathrm{H}$ NMR analysis using 1,3,5-trimethoxybenzene as internal standard. 
Effect of Base<smiles></smiles>

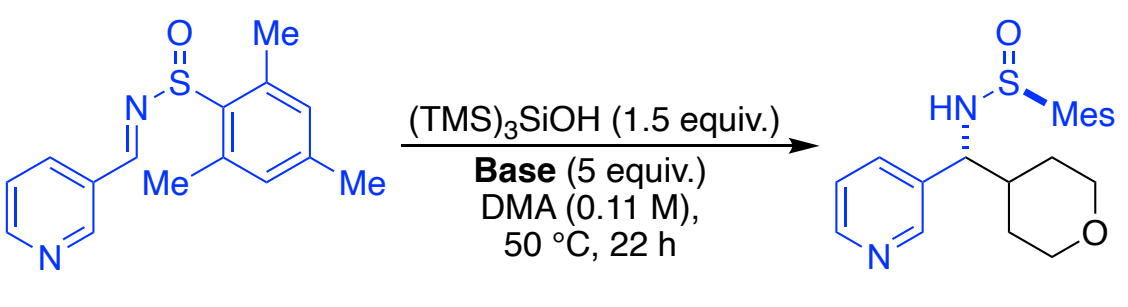

\begin{tabular}{ccc} 
entry & base & yield $(\%)^{a}$ \\
\hline 1 & $\mathrm{~K}_{2} \mathrm{CO}_{3}$ & 50 \\
2 & $\mathrm{Cs}_{2} \mathrm{CO}_{3}$ & 28 \\
3 & $\mathrm{Na}_{2} \mathrm{CO}_{3}$ & 11 \\
4 & $\mathrm{Li}_{2} \mathrm{CO}_{3}$ & 2 \\
5 & Hantzsch Ester & 3 \\
6 & $\mathrm{NEt}_{3}$ & 0 \\
7 & 2,6-lutidine & 0
\end{tabular}

${ }^{a}$ Determined by ${ }^{1} \mathrm{H}$ NMR analysis using 1,3,5-trimethoxybenzene as internal standard. 


\section{Effect of Base Equivalents}

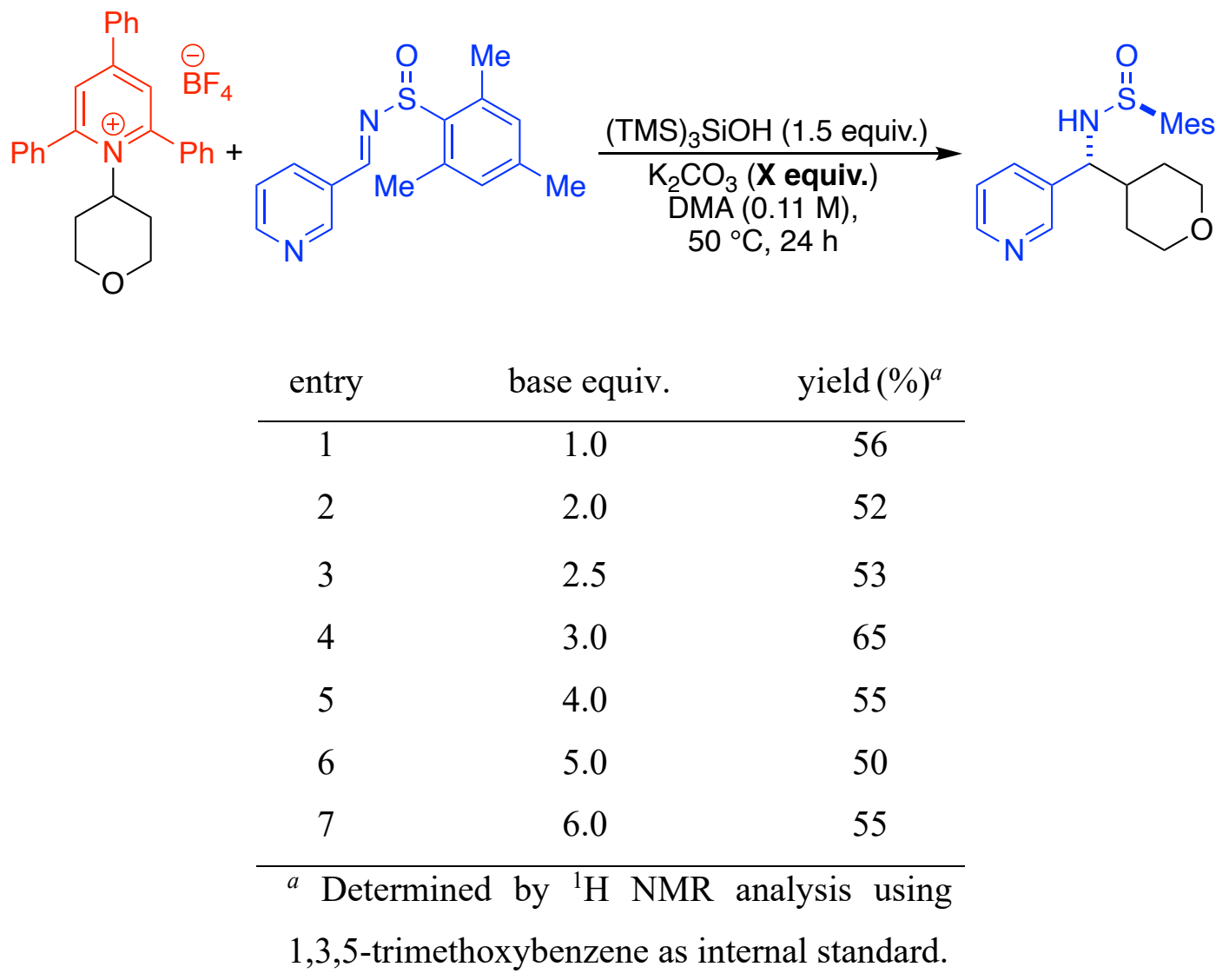




\section{Limitations in Scope}

\section{Representative Sulfinimine Limitations}

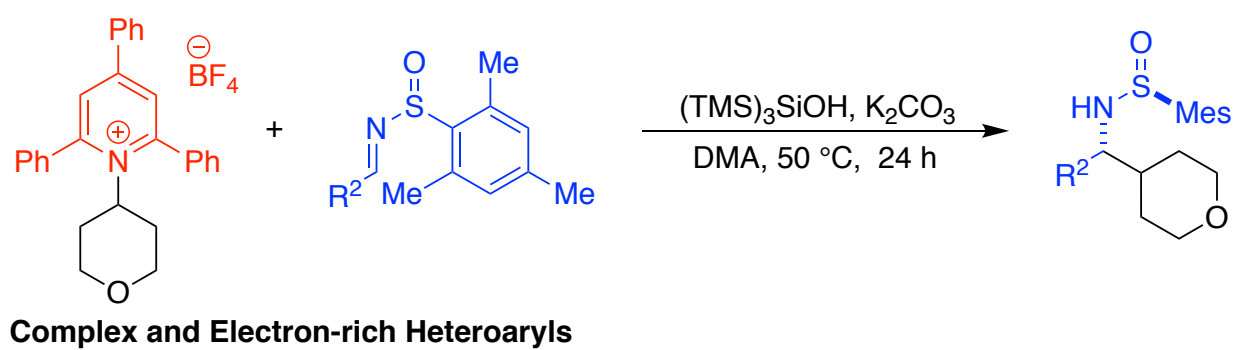

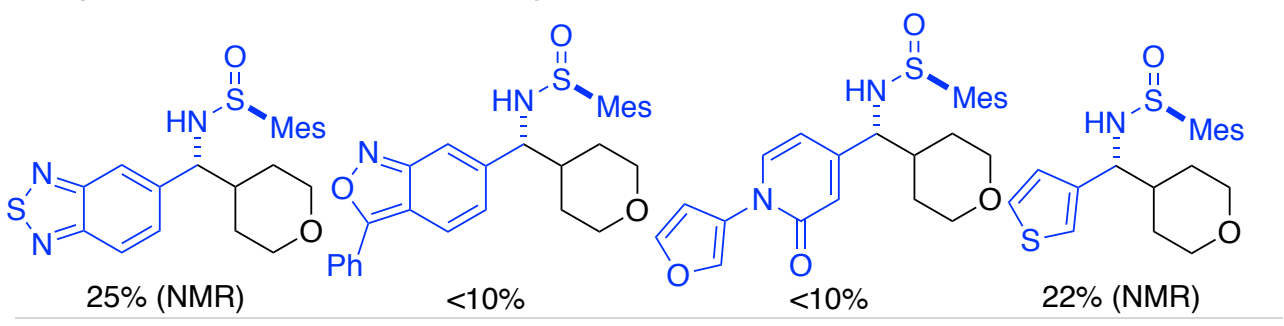<smiles>CCO[Mg]O[Mg]</smiles><smiles>CN(C)c1ccc(C(NS(C)=O)C2CCOCC2)cc1</smiles>

Aliphatic<smiles>CS(=O)NC(C1CCCCC1)C1CCOCC1</smiles>

Although aryl chlorides and aryl fluorides were tolerated in this method, aryl bromides underwent protodehalogenation under these reaction conditions.<smiles>CC(C)N(c1ccccc1)c1cc(-c2ccccc2)cc(-c2ccccc2)c1NS(C)=O</smiles>

$p$-Tolyl was not successful when used in place of the mesityl group on the chiral auxiliary.<smiles></smiles>

2.5 equiv<smiles>Cc1ccc(S(=O)/N=C/c2cccnc2)cc1</smiles>

1.0 equiv

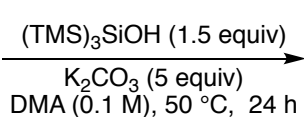

$\operatorname{DMA}(0.1 \mathrm{M}), 50^{\circ} \mathrm{C}, 24 \mathrm{~h}$

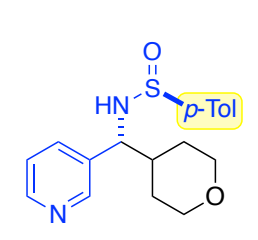

$0 \%$ (NMR)<smiles>Cc1ccccc1</smiles>

$10 \%(\mathrm{NMR})^{a}$

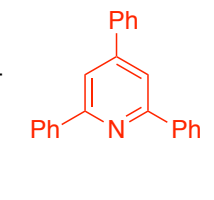

$69 \%(\mathrm{NMR})^{a}$

\footnotetext{
a Yield based on amount of pyridinium starting material used. Maximum yield is $100 \%$ (not 250\%).
} 


\section{Representative Pyridinium Salt Limitations}

As shown below, primary alkylpyridinium salts resulted in no desired product. We also observed low yield with a pyridinium salt derived from indomethacin.
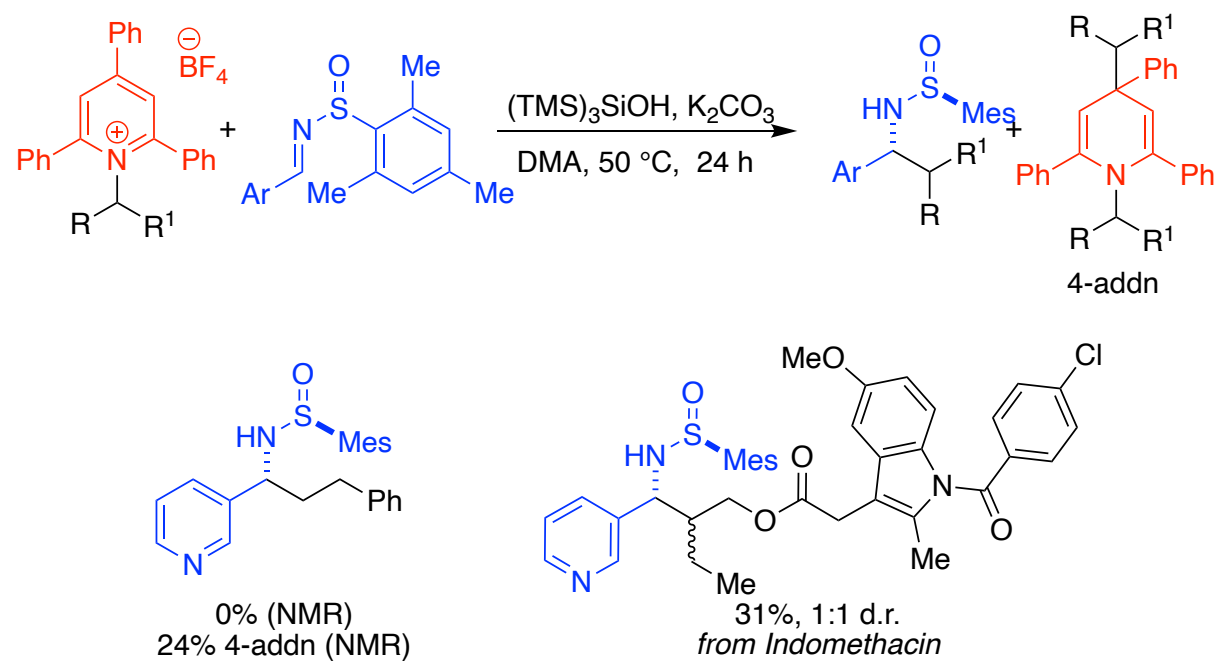


\section{Radical Addition of Alkylpyridinium Salts to Sulfinimines}

General Procedure A: Coupling of Alkylpyridinium Salts and Sulfinimines

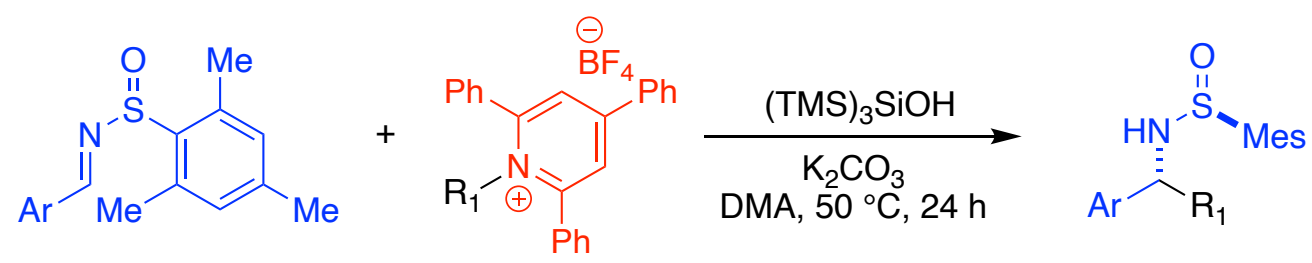

An oven-dried, 25-mL Schlenk flask equipped with a mechanical stir bar was charged with alkylpyridinium salt (2.5 mmol, 2.5 equiv), sulfinimine (1.0 mmol, 1.0 equiv), and $\mathrm{K}_{2} \mathrm{CO}_{3}(0.42$ g, $3.0 \mathrm{mmol}, 3.0$ equiv). The flask was fitted with a rubber septum, sealed with parafilm, and evacuated and backfilled with nitrogen three times. Dimethylacetamide (DMA, $9.0 \mathrm{~mL}$ ) and tris(trimethylsilyl)silanol $(0.41 \mathrm{~mL}, 1.5 \mathrm{mmol}, 1.5$ equiv) were added via syringe, and the mixture was stirred at $50{ }^{\circ} \mathrm{C}$ for $24 \mathrm{~h}$ in an oil bath. The mixture was allowed to cool to room temperature. The mixture was filtered through Celite ${ }^{\circledR}$ with EtOAc $(50 \mathrm{~mL})$. The organic layer was washed with 1:1 $\mathrm{H}_{2} \mathrm{O}$ :brine ( 4 x $50 \mathrm{~mL}$ ), dried with $\mathrm{MgSO}_{4}$, filtered, and concentrated. The product was then purified via silica gel chromatography.

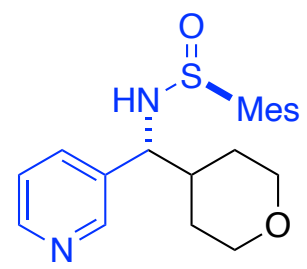

\section{2,4,6-Trimethyl- $N$-[2-methyl-1-(3-pyridyl)tetrahydro-2H-pyran-4-yl]benzenesulfinamide}

(3). Prepared via General Procedure A using alkylpyridinium salt 1a and sulfinimine 2a. The crude mixture was purified by silica gel chromatography $(1 \% \rightarrow 5 \% \rightarrow 12 \% \mathrm{MeOH} / \mathrm{DCM})$ to give 3 (359 mg, 71\%, >20:1 dr) as a white solid (mp: $\left.141-144{ }^{\circ} \mathrm{C}\right):{ }^{1} \mathrm{H}$ NMR $\left(400 \mathrm{MHz}, \mathrm{CDCl}_{3}\right) \delta 8.54$ $-8.40(\mathrm{~m}, 2 \mathrm{H}), 7.53(\mathrm{dt}, J=7.9,2.0 \mathrm{~Hz}, 1 \mathrm{H}), 7.23(\mathrm{dd}, J=7.9,4.8 \mathrm{~Hz}, 1 \mathrm{H}), 6.79(\mathrm{~s}, 2 \mathrm{H}), 4.51$ (d, $J=6.4 \mathrm{~Hz}, 1 \mathrm{H}), 4.23(\mathrm{t}, J=7.0 \mathrm{~Hz}, 1 \mathrm{H}), 4.06-3.96(\mathrm{~m}, 1 \mathrm{H}), 3.91(\mathrm{~m}, 1 \mathrm{H}), 3.32(\mathrm{dtd}, J=25.7$, 11.7, 2.6 Hz, 2H), 2.48 (s, 6H), 2.25 (s, 3H), 2.08-2.00 (m, 1H), $1.87-1.82(\mathrm{~m}, 1 \mathrm{H}), 1.39-1.22$ (m, 3H).; ${ }^{13} \mathrm{C}$ NMR $\left(151 \mathrm{MHz}, \mathrm{CDCl}_{3}\right) \delta 149.1,148.9,141.1,137.6,136.7,136.3,135.1,131.1$, 123.5, 67.8, 67.6, 62.1, 40.8, 30.0, 29.6, 21.1, 19.6.; FTIR (thin film) 3207, 2949, 2845, 2361, 2336, 1652, 1456, 1091, 715, $667 \mathrm{~cm}^{-1}$; HRMS (ESI+) $[\mathrm{M}+\mathrm{H}]^{+}$calculated for $\mathrm{C}_{20} \mathrm{H}_{27} \mathrm{~N}_{2} \mathrm{O}_{2} \mathrm{~S}$ : 359.1793 , found 359.1781 . 
Reaction with “Minimal Precaution” Set-up

Product 3 was also prepared via a procedure similar to General Procedure A, except that minimal precautions were taken to protect the reaction from air and moisture. Dimethylacetamide was used as received; it was not dried or degassed. The round-bottomed flask and stir bar were not oven-dried prior to use. Reagents were added directly into the flask, open to air. The reaction was set up and run under air (no $\left.\mathrm{N}_{2}\right)$.

To a $25-\mathrm{mL}$ round bottomed flask equipped with a stir bar was added alkylpyridinium salt $1 \mathbf{a}$ (1.2 g, $2.5 \mathrm{mmol}, 2.5$ equiv), sulfinimine $2 \mathbf{a}$ (272 mg, $1.0 \mathrm{mmol}, 1.0$ equiv), and $\mathrm{K}_{2} \mathrm{CO}_{3}(0.42$ g, $3.0 \mathrm{mmol}, 3.0$ equiv). Dimethylacetamide (DMA, $9.0 \mathrm{~mL}$ ) and tris(trimethylsilyl)silanol (0.41 $\mathrm{mL}, 1.5 \mathrm{mmol}, 1.5$ equiv) were added via syringe. The flask was fitted with a rubber septum and sealed with parafilm (no $\mathrm{N}_{2}$ inlet). The resulting mixture was stirred at $50{ }^{\circ} \mathrm{C}$ for $24 \mathrm{~h}$ in an oil bath. The mixture was allowed to cool to room temperature. The mixture was filtered through Celite ${ }^{\circledR}$ with EtOAc $(50 \mathrm{~mL})$. The organic layer was washed with 1:1 $\mathrm{H}_{2} \mathrm{O}$ :brine $(4 \mathrm{x} 50 \mathrm{~mL})$, dried with $\mathrm{MgSO}_{4}$, filtered, and concentrated. The crude mixture was then purified via silica gel chromatography $(1 \% \rightarrow 5 \% \rightarrow 12 \% \mathrm{MeOH} / \mathrm{DCM})$ to give $3(147 \mathrm{mg}, 41 \%,>20: 1 \mathrm{dr})$.

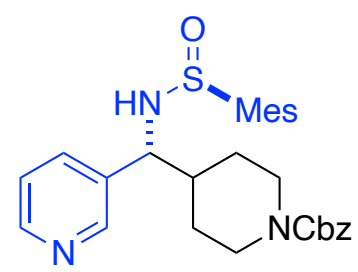

\section{2,4,6-Trimethyl- $N$-[2-methyl-1-(3-pyridyl)(4-N-Cbz-piperdine)]benzenesulfinamide}

(4).

Prepared via General Procedure A using alkylpyridinium salt $\mathbf{1 b}$ and sulfinimine 2a. The crude mixture was purified by silica gel chromatography $(50 \% \rightarrow 100 \%$ EtOAc/hexanes) to give 4 (258 $\mathrm{mg}, 52 \%,>20: 1 \mathrm{dr})$ as a light yellow solid $\left(\mathrm{mp}: 82-84{ }^{\circ} \mathrm{C}\right):{ }^{1} \mathrm{H} \mathrm{NMR}\left(600 \mathrm{MHz}, \mathrm{CDCl}_{3}\right) \delta 8.50$ - $8.49(\mathrm{~m}, 1 \mathrm{H}), 8.44(\mathrm{~s}, 1 \mathrm{H}), 7.52(\mathrm{~m}, 1 \mathrm{H}), 7.37-7.28(\mathrm{~m}, 5 \mathrm{H}), 7.23(\mathrm{dd}, J=7.9,4.8 \mathrm{~Hz}, 1 \mathrm{H})$, $6.79(\mathrm{~s}, 2 \mathrm{H}), 5.08(\mathrm{~s}, 2 \mathrm{H}), 4.54(\mathrm{~d}, J=6.3 \mathrm{~Hz}, 1 \mathrm{H}), 4.29-4.08(\mathrm{~m}, 3 \mathrm{H}), 2.70(\mathrm{~m}, 2 \mathrm{H}), 2.48(\mathrm{~s}$, 6H), 2.25 (s, 3H), 1.99 (dt, $J=11.2,7.0,3.3 \mathrm{~Hz}, 1 \mathrm{H}), 1.94-1.92(\mathrm{~m}, 1 \mathrm{H}), 1.47-1.45(\mathrm{~m}, 1 \mathrm{H})$, $1.23-1.14(\mathrm{~m}, 1 \mathrm{H}), 1.10(\mathrm{~m}, 1 \mathrm{H}) ;{ }^{13} \mathrm{C} \mathrm{NMR}\left(151 \mathrm{MHz}, \mathrm{CDCl}_{3}\right) \delta 155.2,149.1,148.7,141.1$, $137.4,136.9,136.7,136.3,135.2$, 131.1, 128.6, 128.1, 128.0, 123.6, 67.2, 61.7, 44.0, 43.8, 41.6, 
21.1, 19.6; FTIR (thin film) 3016, 1739, 1699, 1635, 1575, 1456, 1083, $671 \mathrm{~cm}^{-1}$; HRMS (ESI+) $[\mathrm{M}+\mathrm{H}]^{+}$calculated for $\mathrm{C}_{28} \mathrm{H}_{34} \mathrm{~N}_{3} \mathrm{O}_{3} \mathrm{~S}: 492.2321$, found 492.2313 .

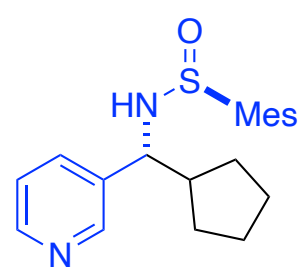

2,4,6-Trimethyl- $N$-[2-methyl-1-(3-pyridyl)cyclopentyl]benzenesulfinamide (5). Prepared via General Procedure A using alkylpyridinium salt $\mathbf{1 c}$ and sulfinimine 2a. The crude mixture was purified by silica gel chromatography $(20 \% \rightarrow 40 \%$ EtOAc/hexanes) to give 5 (178 mg, 52\%, $>20: 1 \mathrm{dr})$ as a light yellow solid (mp: $\left.103-105{ }^{\circ} \mathrm{C}\right):{ }^{1} \mathrm{H}$ NMR $\left(600 \mathrm{MHz}, \mathrm{CDCl}_{3}\right) \delta 8.45(\mathrm{dt}, J=$ 6.4, $2.1 \mathrm{~Hz}, 2 \mathrm{H}), 7.57$ (dt, $J=7.8,2.0 \mathrm{~Hz}, 1 \mathrm{H}), 7.18$ (dd, $J=7.9,4.5 \mathrm{~Hz}, 1 \mathrm{H}), 6.74(\mathrm{~s}, 2 \mathrm{H}), 4.53$ (d, $J=5.8 \mathrm{~Hz}, 1 \mathrm{H}), 4.23$ (ddd, $J=8.8,5.6,2.4 \mathrm{~Hz}, 1 \mathrm{H}), 2.48$ (s, 6H), 2.35 (q, $J=8.5 \mathrm{~Hz}, 1 \mathrm{H}$ ), $2.22(\mathrm{~s}, 3 \mathrm{H}), 1.94(\mathrm{dt}, J=12.2,6.1 \mathrm{~Hz}, 1 \mathrm{H}), 1.64(\mathrm{dd}, J=12.3,4.7 \mathrm{~Hz}, 1 \mathrm{H}), 1.61-1.54(\mathrm{~m}, 2 \mathrm{H})$, $1.53-1.41(\mathrm{~m}, 3 \mathrm{H}), 1.11(\mathrm{dq}, J=12.6,8.2 \mathrm{~Hz}, 1 \mathrm{H}) ;{ }^{13} \mathrm{C} \mathrm{NMR}\left(151 \mathrm{MHz}, \mathrm{CDCl}_{3}\right) \delta 148.9,148.7$, 140.8, 138.0, 137.5, 136.7, 135.0, 131.0, 123.4, 61.6, 46.5, 30.6, 30.1, 25.4, 25.3, 21.1, 19.6; FTIR (thin film) 3194, 2954, 2916, 2848, 1425, 1072, 1048, 849, $715 \mathrm{~cm}^{-1}$; HRMS (ESI+) $[\mathrm{M}+\mathrm{H}]^{+}$ calculated for $\mathrm{C}_{20} \mathrm{H}_{27} \mathrm{~N}_{2} \mathrm{OS}: 343.1844$, found 343.1833 .

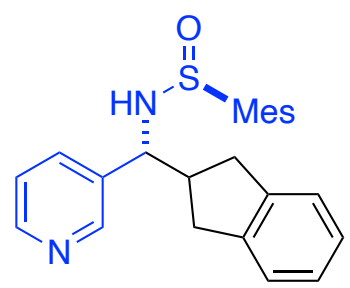

2,4,6-Trimethyl- $N$-[2-methyl-1-(3-pyridyl)indanyl]benzenesulfinamide (6). Prepared via General Procedure A using alkylpyridinium salt 1d and sulfinimine 2a. The crude mixture was purified by silica gel chromatography $(20 \% \rightarrow 40 \%$ EtOAc/hexanes) to give 6 (189 mg, 48\%, $>20: 1 \mathrm{dr}$ ) as a brown solid (mp: $\left.106-108{ }^{\circ} \mathrm{C}\right):{ }^{1} \mathrm{H}$ NMR $\left(400 \mathrm{MHz}, \mathrm{CDCl}_{3}\right) \delta 8.50(\mathrm{~m}, 2 \mathrm{H}), 7.63$ $(\mathrm{d}, J=7.9 \mathrm{~Hz}, 1 \mathrm{H}), 7.23-7.06(\mathrm{~m}, 5 \mathrm{H}), 6.77(\mathrm{~s}, 2 \mathrm{H}), 4.58(\mathrm{~d}, J=6.2 \mathrm{~Hz}, 1 \mathrm{H}), 4.48(\mathrm{t}, J=7.3 \mathrm{~Hz}$, 1H), $3.26-3.15$ (m, 1H), $3.05-2.94$ (m, 2H), 2.75 (dd, $J=15.9,7.4 \mathrm{~Hz}, 1 \mathrm{H}), 2.64$ (s, 1H), 2.49 (s, 6H), 2.24 (s, 3H); ${ }^{13} \mathrm{C}$ NMR (101 MHz, $\left.\mathrm{CDCl}_{3}\right) \delta 148.9,148.6,142.0,141.0,137.6,137.5$, 136.7, 135.1, 131.1, 126.74, 126.71, 124.68, 124.5, 123.7, 123.6, 61.3, 45.9, 37.0, 36.8, 21.1, 19.6; FTIR (thin film) 2926, 1071, 1047, 850, 744, $715 \mathrm{~cm}^{-1}$; HRMS (ESI+) $[\mathrm{M}+\mathrm{H}]^{+}$calculated for $\mathrm{C}_{24} \mathrm{H}_{27} \mathrm{~N}_{2} \mathrm{OS}: 391.1844$, found 391.1828 . 


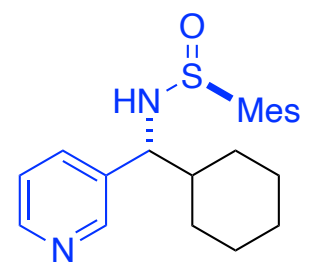

2,4,6-Trimethyl- $N$-[2-methyl-1-(3-pyridyl)cyclohexyl]benzenesulfinamide (7). Prepared via General Procedure A using alkylpyridinium salt 1e and sulfinimine 2a. The crude mixture was purified by silica gel chromatography $(0 \% \rightarrow 10 \% \mathrm{MeOH} / \mathrm{DCM})$ to give $7(227 \mathrm{mg}, 64 \%,>20: 1$ dr) as a white solid (mp: $\left.115-116{ }^{\circ} \mathrm{C}\right)$ : ${ }^{1} \mathrm{H}$ NMR $\left(600 \mathrm{MHz}, \mathrm{CDCl}_{3}\right) \delta 8.46(\mathrm{~d}, J=4.7 \mathrm{~Hz}, 1 \mathrm{H})$, 8.42 (s, 1H), $7.51(\mathrm{dt}, J=7.9,2.0 \mathrm{~Hz}, 1 \mathrm{H}), 7.19$ (dd, $J=7.9,4.8 \mathrm{~Hz}, 1 \mathrm{H}), 6.76(\mathrm{~s}, 2 \mathrm{H}), 4.50$ (d, $J$ $=5.9 \mathrm{~Hz}, 1 \mathrm{H}), 4.25(\mathrm{t}, J=6.4 \mathrm{~Hz}, 1 \mathrm{H}), 2.50(\mathrm{~s}, 6 \mathrm{H}), 2.23(\mathrm{~s}, 3 \mathrm{H}), 1.91(\mathrm{dt}, J=12.7,3.3 \mathrm{~Hz}, 1 \mathrm{H})$, $1.84-1.72(\mathrm{~m}, 2 \mathrm{H}), 1.70-1.60(\mathrm{~m}, 2 \mathrm{H}), 1.53(\mathrm{dt}, J=13.1,3.3 \mathrm{~Hz}, 1 \mathrm{H}), 1.25-1.14(\mathrm{~m}, 2 \mathrm{H}), 1.06$ $(\mathrm{qt}, J=12.9,3.6 \mathrm{~Hz}, 1 \mathrm{H}), 0.95-0.82(\mathrm{~m}, 2 \mathrm{H}) ;{ }^{13} \mathrm{C} \mathrm{NMR}\left(151 \mathrm{MHz}, \mathrm{CDCl}_{3}\right) \delta 148.9,148.6,140.9$, 137.6, 137.1, 136.7, 135.2, 131.0, 123.3, 62.0, 43.2, 29.9, 29.3, 26.3, 26.1, 26.0, 21.0, 19.6; FTIR (thin film) 3200, 2924, 2851, 1448, 1425, 1072, 1047, $715 \mathrm{~cm}^{-1}$; HRMS (ESI+) $[\mathrm{M}+\mathrm{H}]^{+}$calculated for $\mathrm{C}_{21} \mathrm{H}_{29} \mathrm{~N}_{2} \mathrm{OS}$ : 357.2001, found 357.1992.

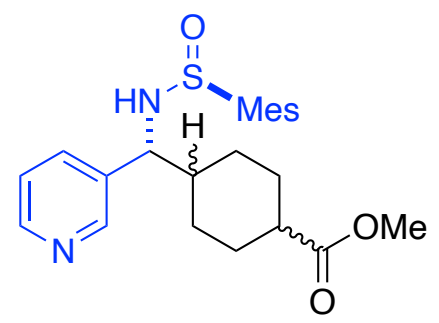

Methyl 4-((1S)-((mesitylsulfinyl)amino)(pyridin-3-yl)methyl)cyclohexane-1-carboxylate (8). Prepared via General Procedure A using alkylpyridinium salt $\mathbf{1} \mathbf{j}$ and sulfinimine $\mathbf{2 a}$. The crude mixture was purified by silica gel chromatography $(75 \% \rightarrow 100 \%$ EtOAc/hexanes) to give 8 as a mixture of diastereomers $(2: 1$ trans:cis, $252 \mathrm{mg}, 61 \%)$ as a white solid (mp: $\left.148-150{ }^{\circ} \mathrm{C}\right):{ }^{1} \mathrm{H}$ NMR (400 MHz, $\mathrm{CDCl}_{3}$, both diastereomers) $\delta 8.45(\mathrm{td}, J=4.7,1.6 \mathrm{~Hz}, 1 \mathrm{H}), 8.40(\mathrm{dd}, J=4.9$, $2.4 \mathrm{~Hz}, 1 \mathrm{H}), 7.48(\mathrm{dt}, J=8.0,1.9 \mathrm{~Hz}, 1 \mathrm{H}), 7.18(\mathrm{ddd}, J=7.9,4.9,2.9 \mathrm{~Hz}, 1 \mathrm{H}), 6.75(\mathrm{~d}, J=5.8$ $\mathrm{Hz}, 2 \mathrm{H}), 4.58(\mathrm{dd}, J=6.4,4.1 \mathrm{~Hz}, 1 \mathrm{H}), 4.23(\mathrm{td}, J=6.8,2.8 \mathrm{~Hz}, 1 \mathrm{H}), 3.62$ (d, $J=4.6 \mathrm{~Hz}, 3 \mathrm{H})$, $2.46(\mathrm{~s}, 6 \mathrm{H}), 2.22$ (d, $J=3.5 \mathrm{~Hz}, 3 \mathrm{H}), 2.13(\mathrm{tt}, J=12.3,3.4 \mathrm{~Hz}, 1 \mathrm{H}), 2.05-1.90$ (m, 2H), 1.80 (tdd, $J=13.7,6.3,3.1 \mathrm{~Hz}, 1 \mathrm{H}), 1.60(\mathrm{dt}, J=13.0,3.0 \mathrm{~Hz}, 1 \mathrm{H}), 1.44-1.22(\mathrm{~m}, 3 \mathrm{H}), 1.12-0.82$ $(\mathrm{m}, 2 \mathrm{H}) ;{ }^{13} \mathrm{C} \mathrm{NMR}\left(101 \mathrm{MHz}, \mathrm{CDCl}_{3}\right.$, both diastereomers) $\delta 176.1,175.4,148.8,148.75,148.71$, $148.6,140.9,140.8,137.5,137.2,136.6,136.6,135.0,134.9,131.0,130.9,123.4,123.3,61.7$, 
61.2, 51.7, 42.9, 42.2, 42.1, 39.5, 28.9, 28.6, 28.4, 28.1, 26.4, 26.3, 26.1, 26.1, 21.03, 21.02, 19.54, 19.53; FTIR (thin film) 2932, 1732, 1435, 1384, $1083 \mathrm{~cm}^{-1}$; HRMS (ESI+) $[\mathrm{M}+\mathrm{H}]^{+}$calculated for $\mathrm{C}_{23} \mathrm{H}_{31} \mathrm{~N}_{2} \mathrm{O}_{3} \mathrm{~S}: 415.2055$, found 415.2041 .

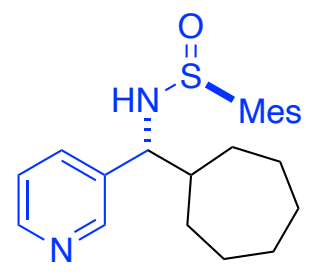

2,4,6-Trimethyl- $N$-[2-methyl-1-(3-pyridyl)cycloheptyl]benzenesulfinamide (9). Prepared via General Procedure A using alkylpyridinium salt 1f and sulfinimine 2a. The crude mixture was purified by silica gel chromatography $(1 \% \rightarrow 10 \% \mathrm{MeOH} / \mathrm{DCM})$ to give $9(321 \mathrm{mg}, 87 \%,>20: 1$ dr) as a light yellow solid (mp: $\left.109-112{ }^{\circ} \mathrm{C}\right):{ }^{1} \mathrm{H} \mathrm{NMR}\left(400 \mathrm{MHz}, \mathrm{CDCl}_{3}\right) \delta 8.46(\mathrm{dd}, J=4.8$, $1.6 \mathrm{~Hz}, 2 \mathrm{H}), 7.55$ (dd, $J=7.9,2.0 \mathrm{~Hz}, 1 \mathrm{H}), 7.18(\mathrm{dd}, J=7.9,4.8 \mathrm{~Hz}, 1 \mathrm{H}), 6.78$ (s, 2H), 4.44 (d, $J$ $=5.2 \mathrm{~Hz}, 1 \mathrm{H}), 4.39(\mathrm{t}, J=5.4 \mathrm{~Hz}, 1 \mathrm{H}), 2.51(\mathrm{~s}, 6 \mathrm{H}), 2.24(\mathrm{~s}, 3 \mathrm{H}), 2.13-2.03(\mathrm{~m}, 1 \mathrm{H}), 1.85-1.73$ (m, 2H), $1.65-1.51$ (m, 5H), 1.42 (dd, $J=10.6,5.6 \mathrm{~Hz}, 3 \mathrm{H}), 1.14$ (dddd, $J=27.4,13.5,8.7,3.4$ $\mathrm{Hz}, 2 \mathrm{H}) ;{ }^{13} \mathrm{C} \mathrm{NMR}\left(101 \mathrm{MHz}, \mathrm{CDCl}_{3}\right) \delta 149.1,148.7,140.9,137.5,136.8,136.7,135.2,131.0$, 123.2, 61.7, 43.8, 31.0, 30.2, 28.2, 27.8, 26.8, 26.7, 21.1, 19.6; FTIR (thin film) 2923, 2853, 1250, $1072,847,758,621 \mathrm{~cm}^{-1}$; HRMS (ESI+) $[\mathrm{M}+\mathrm{H}]^{+}$calculated for $\mathrm{C}_{22} \mathrm{H}_{31} \mathrm{~N}_{2} \mathrm{OS}: 371.2157$, found 371.2141 .

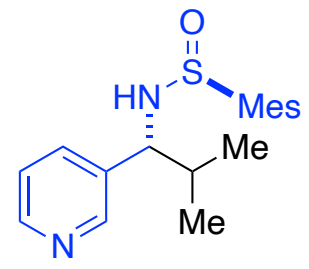

2,4,6-Trimethyl- $N$-[2-methyl-1-(3-pyridyl)propyl]benzenesulfinamide (10). Prepared via General Procedure A using alkylpyridinium salt 1g and sulfinimine 2a. The crude mixture was purified by silica gel chromatography $(20 \% \rightarrow 50 \%$ EtOAc/hexanes) to give $\mathbf{1 0}$ ( $278 \mathrm{mg}, 88 \%$, $>20: 1 \mathrm{dr})$ as a red oil: ${ }^{1} \mathrm{H}$ NMR $\left(600 \mathrm{MHz}, \mathrm{CDCl}_{3}\right) \delta 8.47(\mathrm{dd}, J=4.9,1.6 \mathrm{~Hz}, 1 \mathrm{H}), 8.45(\mathrm{~d}, J=$ $2.3 \mathrm{~Hz}, 1 \mathrm{H}), 7.54(\mathrm{dt}, J=7.9,2.1 \mathrm{~Hz}, 1 \mathrm{H}), 7.20(\mathrm{dd}, J=7.9,4.8 \mathrm{~Hz}, 1 \mathrm{H}), 6.78$ (s, 2H), $4.48(\mathrm{~d}, J$ $=5.6 \mathrm{~Hz}, 1 \mathrm{H}), 4.27(\mathrm{t}, J=6.0 \mathrm{~Hz}, 1 \mathrm{H}), 2.51(\mathrm{~s}, 6 \mathrm{H}), 2.24(\mathrm{~s}, 3 \mathrm{H}), 2.22-2.18(\mathrm{~m}, 1 \mathrm{H}), 0.96(\mathrm{~d}, J$ $=6.7 \mathrm{~Hz}, 3 \mathrm{H}), 0.84(\mathrm{~d}, J=6.6 \mathrm{~Hz}, 3 \mathrm{H}) ;{ }^{13} \mathrm{C} \mathrm{NMR}\left(151 \mathrm{MHz}, \mathrm{CDCl}_{3}\right) \delta 149.0,148.8,140.9,137.6$, 136.7, 136.6, 135.2, 131.0, 123.2, 62.4, 33.3, 21.1, 19.6, 19.2, 18.6; FTIR (thin film) 3207, 2961, 
2927, 1600,1382, 1073, 1049, 1025, 849, $715 \mathrm{~cm}^{-1}$; HRMS $(\mathrm{ESI}+)[\mathrm{M}+\mathrm{H}]^{+}$calculated for $\mathrm{C}_{18} \mathrm{H}_{25} \mathrm{~N}_{2} \mathrm{OS}: 317.1688$, found 317.1676 .

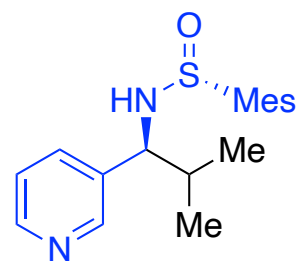

$(R)-2,4,6-$ Trimethyl- $N$-[(R)-2-methyl-1-(3-pyridyl)propyl]benzenesulfinamide $\quad((R, R)-10)$. Prepared via General Procedure A on a $0.5 \mathrm{mmol}$ scale using alkylpyridinium salt $\mathbf{1 g}$ and enantiopure sulfinimine 2a. The crude mixture was purified by silica gel chromatography $(20 \% \rightarrow$ $50 \%$ EtOAc/hexanes) to give $(\boldsymbol{R}, \boldsymbol{R})-\mathbf{1 0}(133 \mathrm{mg}, 84 \%,>20: 1 \mathrm{dr})$ as a white solid that had analytical data consistent with that noted above. The enantiomeric excess was determined to be $>99 \%$ by chiral SFC analysis (CHIRALPAK IA-3, $2.5 \mathrm{~mL} / \mathrm{min}, 15 \% \mathrm{MeOH}$ in $\mathrm{CO}_{2}, \lambda=254 \mathrm{~nm}$ ); $t_{\mathrm{R}}$ (major) $=1.055 \mathrm{~min}, t_{\mathrm{R}}($ minor $)=1.4133 \mathrm{~min} .[\alpha]_{D}^{22}=-88.5\left(\mathrm{c}=1.42, \mathrm{CHCl}_{3}\right)$.

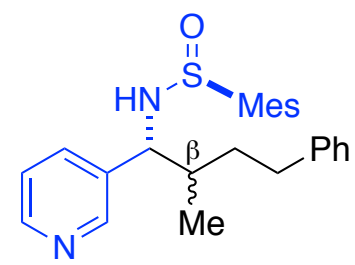

\section{2,4,6-Trimethyl- $N$-[2-methyl-1-(3-pyridyl)(1-methyl-propylbenzene)]benzenesulfinamide}

(11). Prepared via General Procedure A using alkylpyridinium salt 1i and sulfinimine 2a. The crude mixture was purified by silica gel chromatography $(5 \% \rightarrow 40 \%$ EtOAc/hexanes) to give $\mathbf{1 1}$ as a mixture of inseparable diastereomers (1:1.1 d.r. at $\left.\mathrm{C}_{\beta}, 260 \mathrm{mg}, 64 \%\right)$ as a red solid (mp: $98-$ $\left.102{ }^{\circ} \mathrm{C}\right):{ }^{1} \mathrm{H} \mathrm{NMR}\left(600 \mathrm{MHz}, \mathrm{CDCl}_{3}\right.$, both diastereomers) $\delta 8.38-8.30(\mathrm{~m}, 2 \mathrm{H}), 7.43(\mathrm{t}, J=9.3$ $\mathrm{Hz}, 1 \mathrm{H}), 7.15\left(\mathrm{~m}, 2 \mathrm{H}\right.$, overlapping with $\left.\mathrm{CHCl}_{3}\right), 7.12-7.05(\mathrm{~m}, 2 \mathrm{H}), 7.03-6.96(\mathrm{~m}, 2 \mathrm{H}), 6.69-$ $6.65(\mathrm{~m}, 2 \mathrm{H}), 4.39-4.27$ (m, 2H), $2.67-2.57$ (m, 1H), 2.46 (ddd, $J=15.1,8.6,4.5 \mathrm{~Hz}, 1 \mathrm{H}), 2.40$ (m, 6H), $2.14(\mathrm{~m}, 3 \mathrm{H}), 2.04-1.92(\mathrm{~m}, 1 \mathrm{H}), 1.71(\mathrm{ddtd}, J=24.3,14.0,6.6,3.6 \mathrm{~Hz}, 1 \mathrm{H}), 1.23(\mathrm{dqd}$, $J=14.0,9.1,5.4 \mathrm{~Hz}, 1 \mathrm{H}), 0.87-0.81(\mathrm{~m}, 3 \mathrm{H}) ;{ }^{13} \mathrm{C} \mathrm{NMR}\left(101 \mathrm{MHz}, \mathrm{CDCl}_{3}\right.$, both diastereomers) $\delta$ 148.9, 148.75, 148.74, 148.6, 142.0, 141.9, 141.0, 137.5, 137.4, 136.8, 136.7, 136.6, 136.3, 135.4, 135.2, 131.0, 128.6, 128.4, 126.1, 126.0, 123.3, 123.2, 60.9, 60.8, 37.7, 37.5, 35.0, 34.3, 33.4, 33.4, 
21.1, 19.6, 15.7, 15.4; FTIR (thin film) 3025, 2922, 1383, $1083 \mathrm{~cm}^{-1}$; HRMS (ESI+) $[\mathrm{M}+\mathrm{H}]^{+}$ calculated for $\mathrm{C}_{25} \mathrm{H}_{31} \mathrm{~N}_{2} \mathrm{OS}$ : 407.2157, found 407.2140.

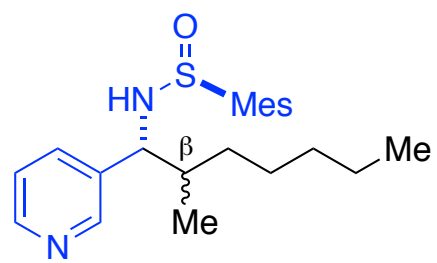

2,4,6-Trimethyl- $N$-[2-methyl-1-(3-pyridyl)(sec-heptyl)]benzenesulfinamide (12). Prepared via General Procedure A using alkylpyridinium salt $\mathbf{1 h}$ and sulfinimine 2a. The crude mixture was purified by silica gel chromatography $(5 \% \rightarrow 50 \%$ EtOAc/hexanes) to give $\mathbf{1 2}$ as a mixture of inseparable diastereomers $\left(1: 1.2\right.$ d.r. at $\left.\mathrm{C}_{\beta}, 242 \mathrm{mg}, 65 \%\right)$ as a yellow solid (mp: $138-141^{\circ} \mathrm{C}$ ): ${ }^{1} \mathrm{H}$ NMR (600 MHz, $\mathrm{CDCl}_{3}$, both diastereomers) $\delta 8.30-8.26$ (m, 2H), 7.37 (ddt, $J=14.8,7.9$, $2.0 \mathrm{~Hz}, 1 \mathrm{H}), 7.01(\mathrm{dt}, J=8.3,4.4 \mathrm{~Hz}, 1 \mathrm{H}), 6.60(\mathrm{~s}, 2 \mathrm{H}), 4.25(\mathrm{~m}, 1 \mathrm{H}), 4.21(\mathrm{q}, J=5.7 \mathrm{~Hz}, 1 \mathrm{H})$, $2.34(\mathrm{~d}, J=3.6 \mathrm{~Hz}, 6 \mathrm{H}), 2.06(\mathrm{~s}, 3 \mathrm{H}), 1.91-1.79(\mathrm{~m}, 1 \mathrm{H}), 1.33-1.17(\mathrm{~m}, 2 \mathrm{H}), 1.08-1.01(\mathrm{~m}$, 5H), 0.82 (dddd, $J=17.4,12.2,9.4,4.5 \mathrm{~Hz}, 1 \mathrm{H}), 0.7-0.64(\mathrm{~m}, 6 \mathrm{H}) ;{ }^{13} \mathrm{C} \mathrm{NMR}\left(151 \mathrm{MHz}, \mathrm{CDCl}_{3}\right.$, both diastereomers) $\delta 149.1,148.9,148.8,148.7,140.9,140.9,137.8,137.6,137.1,136.8,136.7$, 136.5, 135.3, 135.1, 131.05, 131.04, 123.2, 123.2, 61.2, 61.1, 38.6, 38.0, 33.1, 32.7, 32.2, 32.1, 26.9, 26.8, 22.74, 22.73, 21.1, 19.6, 15.6, 15.4, 14.18, 14.17; FTIR (thin film) 2956, 2927, 1465, 1383, $1083 \mathrm{~cm}^{-1}$; HRMS (ESI+) [M+H] ${ }^{+}$calculated for $\mathrm{C}_{22} \mathrm{H}_{33} \mathrm{~N}_{2} \mathrm{OS}: 373.2314$, found 373.2299 .

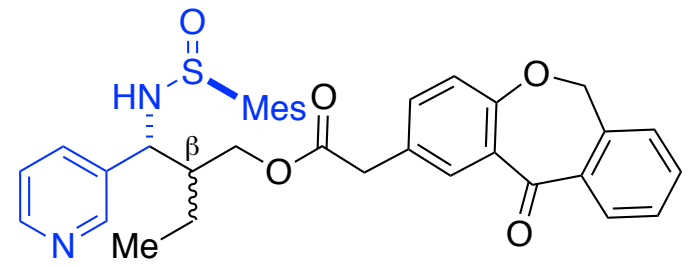

(2S)-2-((1S)-((mesitylsulfinyl)amino)(pyridine-3-yl)methyl)butyl

2-(11-0xo-6, 11dihydrodibenzo[b,e $]$ oxepin-2-yl)acetate (13). Prepared via General Procedure A using alkylpyridinium salt $\mathbf{1 1}$ and sulfinimine 2a. The crude mixture was purified by silica gel chromatography $(50 \% \rightarrow 80 \%$ EtOAc/hexanes) to give $\mathbf{1 3}$ as a mixture of diastereomers (1:1 d.r. at $\left.\mathrm{C}_{\beta}, 262 \mathrm{mg}, 44 \%\right)$ as a white solid (mp: $\left.97-102{ }^{\circ} \mathrm{C}\right):{ }^{1} \mathrm{H} \mathrm{NMR}\left(400 \mathrm{MHz}, \mathrm{CDCl}_{3}\right.$, both diastereomers) $\delta 8.51-8.36(\mathrm{~m}, 2 \mathrm{H}), 8.13(\mathrm{~m}, 1 \mathrm{H}), 7.88(\mathrm{dt}, J=7.8,1.8 \mathrm{~Hz}, 1 \mathrm{H}), 7.58-7.55(\mathrm{~m}$, 1H), $7.48-7.44$ (m, 2H), $7.41-7.34$ (m, 2H), 7.19 (ddd, $J=15.9,7.8,4.8 \mathrm{~Hz}, 1 \mathrm{H}), 7.05$ (dd, $J=$ 8.4, 3.6 Hz, 1H), $6.74(\mathrm{~d}, J=6.7 \mathrm{~Hz}, 2 \mathrm{H}), 5.19(\mathrm{~s}, 2 \mathrm{H}), 4.84-4.68(\mathrm{~m}, 1 \mathrm{H}), 4.57-4.41(\mathrm{~m}, 1 \mathrm{H})$, $4.35-4.12(\mathrm{~m}, 1 \mathrm{H}), 4.10-3.89(\mathrm{~m}, 1 \mathrm{H}), 3.60-3.57(\mathrm{~m}, 2 \mathrm{H}), 2.47-2.41(\mathrm{~m}, 6 \mathrm{H}), 2.20(\mathrm{~d}, J=$ 
$6.2 \mathrm{~Hz}, 3 \mathrm{H}), 2.07(\mathrm{tt}, J=9.9,5.0 \mathrm{~Hz}, 1 \mathrm{H}), 1.24-1.04(\mathrm{~m}, 2 \mathrm{H}), 0.89(\mathrm{t}, J=7.4 \mathrm{~Hz}, 3 \mathrm{H}) ;{ }^{13} \mathrm{C} \mathrm{NMR}$ (101 MHz, $\mathrm{CDCl}_{3}$, both diastereomers) $\delta 190.8,171.3,171.1,160.7,148.7,148.6,5,148.58,148,3$, 141.0, 140.9, 140.89, 140.5, 140.4, 137.6, 137.4, 137.0, 136.8, 136.6, 136.5, 136.4, 136.3, 135.7, 135.2 , 134.9, 133.0, 132.6, 131.0, 130.9, 129.6, 129.4, 128.0, 127.6, 127.56, 127.2, 125.3, 123.5, 123.4, 121.4, 121.3 73.8, 63.8, 63.4, 58.2, 58.0, 45.2, 44.8, 40.4, 34.0, 25.7, 25.1, 21.1, 21.0, 20.1, 19.6, 19.4, 11.8, 11.5; FTIR (thin film) 2924, 1734, 1646, 1384, 1300, $1083 \mathrm{~cm}^{-1}$; HRMS (ESI+) $[\mathrm{M}+\mathrm{H}]^{+}$calculated for $\mathrm{C}_{35} \mathrm{H}_{37} \mathrm{~N}_{2} \mathrm{O}_{5} \mathrm{~S}: 597.2423$, found 597.2413.

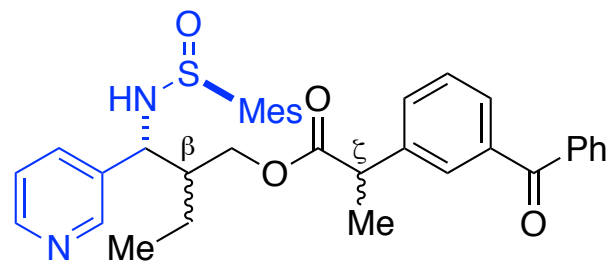

(2S)-2-((1S)-((mesitylsulfinyl)amino)(pyridin3-yl)methyl)butyl

benzoylphenyl)propanoate (14). Prepared via General Procedure A using alkylpyridinium salt $1 \mathbf{k}$ and sulfinimine 2a. The crude mixture was purified by silica gel chromatography $(50 \% \rightarrow$ $100 \%$ EtOAc/hexanes) to give 14 as a mixture of inseparable diastereomers (1:1:1:1 d.r. at $\mathrm{C}_{\beta}$ and $\left.\mathrm{C}_{\zeta}, 274 \mathrm{mg}, 47 \%\right)$ as a light yellow oil: ${ }^{1} \mathrm{H} \mathrm{NMR}\left(400 \mathrm{MHz}, \mathrm{CDCl}_{3}\right.$, both diastereomers) $\delta 8.51-$ $8.33(\mathrm{~m}, 2 \mathrm{H}), 7.80-7.74(\mathrm{~m}, 3 \mathrm{H}), 7.74-7.67(\mathrm{~m}, 1 \mathrm{H}), 7.64-7.58(\mathrm{~m}, 1 \mathrm{H}), 7.51(\mathrm{~m}, 5 \mathrm{H}), 7.25-$ $7.10(\mathrm{~m}, 1 \mathrm{H}), 6.76(\mathrm{~m}, 2 \mathrm{H}), 4.88-4.63(\mathrm{~m}, 1 \mathrm{H}), 4.55-4.36(\mathrm{~m}, 1 \mathrm{H}), 4.32-3.87(\mathrm{~m}, 2 \mathrm{H}), 3.84-$ $3.71(\mathrm{~m}, 1 \mathrm{H}), 2.51-2.38(\mathrm{~m}, 6 \mathrm{H}), 2.23(\mathrm{~m}, 3 \mathrm{H}), 2.06(\mathrm{ddt}, J=15.5,11.0,4.8 \mathrm{~Hz}, 1 \mathrm{H}), 1.59-1.51$ $(\mathrm{m}, 3 \mathrm{H}), 1.34-1.02(\mathrm{~m}, 2 \mathrm{H}), 0.89-0.77(\mathrm{~m}, 3 \mathrm{H}) ;{ }^{13} \mathrm{C} \mathrm{NMR}\left(101 \mathrm{MHz}, \mathrm{CDCl}_{3}\right.$, both diastereomers) $\delta 196.4,174.0,173.9,173.7,173.67,148.7,148.6,148.57,148.5,148.2,148.1$, $140.89,140.86,140.85,140.8,140.69,140.68,140.6,138.1,138.05,138.02,137.42,137.39$, 137.37, 137.3, 136.5, 136.47, 136.45, 136.41, 135.0, 134.7, 132.6, 131.6, 131.5, 131.49, 131.46, $130.91,130.89$, 130.85, 130.08, 130.07, 129.4, 129.31, 129.29, 129.1, 129.09, 129.05, 129.02, 129.0, 128.8, 128.76, 128.74, 128.71, 128.67, 128.6, 128.4, 127.1, 123.4, 123.3, 63.6, 63.4, 63.2, 58.2, 58.0, 57.9, 57.7, 56.0, 45.5, 45.41, 45.39, 45.14, 45.09, 44.7, 44.6, 21.0, 20.9, 20.7, 20.0, 19.96, 19.47, 19.45, 19.4, 19.3, 18.3, 18.13, 18.11, 11.61, 11.58, 11.4; FTIR (thin film) 2965, 2930, 1733, 1653, 1384, 1283, $1083 \mathrm{~cm}^{-1}$; HRMS (ESI+) $[\mathrm{M}+\mathrm{H}]^{+}$calculated for $\mathrm{C}_{35} \mathrm{H}_{39} \mathrm{~N}_{2} \mathrm{O} 4 \mathrm{~S}: 583.2631$, found 583.2616 . 


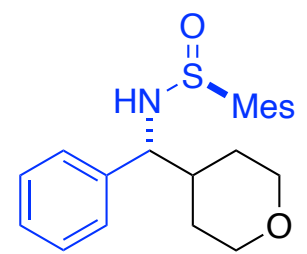

\section{2,4,6-Trimethyl- $N$-[2-methyl-1-(phenyl)tetrahydro-2H-pyran-4-yl)]benzenesulfinamide}

(15). Prepared via General Procedure A using alkylpyridinium salt 1a and sulfinimine 2b. The crude mixture was purified by silica gel chromatography $(20 \% \rightarrow 40 \%$ EtOAc/hexanes) to give 15 (358 mg, 52\%, $>20: 1 \mathrm{dr}$ ) as a white solid that had analytical data consistent with the literature ${ }^{2}$ : ${ }^{1} \mathrm{H}$ NMR $\left(600 \mathrm{MHz}, \mathrm{CDCl}_{3}\right) \delta 7.31(\mathrm{dd}, J=8.3,6.6 \mathrm{~Hz}, 2 \mathrm{H}), 7.27(\mathrm{~s}, 1 \mathrm{H}), 7.22-7.19(\mathrm{~m}, 2 \mathrm{H})$, 6.82 (s, 2H), 4.47 (d, $J=6.0 \mathrm{~Hz}, 1 \mathrm{H}), 4.18$ (t, $J=6.9 \mathrm{~Hz}, 1 \mathrm{H}), 4.02-3.96(\mathrm{~m}, 1 \mathrm{H}), 3.93-3.85$ (m, 1H), 3.36 (td, $J=11.9,2.2 \mathrm{~Hz}, 1 \mathrm{H}), 3.29$ (td, $J=11.7,2.4 \mathrm{~Hz}, 1 \mathrm{H}), 2.49$ (s, 6H), 2.27 (s, 3H), $2.09-2.00(\mathrm{~m}, 1 \mathrm{H}), 1.85(\mathrm{dt}, J=13.0,3.0 \mathrm{~Hz}, 1 \mathrm{H}), 1.38-1.27(\mathrm{~m}, 3 \mathrm{H}) ;{ }^{13} \mathrm{C}$ NMR $(151 \mathrm{MHz}$, $\left.\mathrm{CDCl}_{3}\right) \delta 140.9,140.8,138.4,136.6,131.0,128.8,127.8,127.4,68.0,67.8,64.6,40.8,30.3,29.7$, 21.1, 19.5.

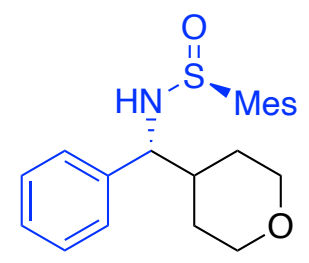

(S)-2,4,6-Trimethyl- $N-[(S)$-2-methyl-1-(phenyl)tetrahydro-2H-pyran-4-

yl)]benzenesulfinamide $((\boldsymbol{S}, S)-15)$. Prepared via General Procedure A on a $0.5 \mathrm{mmol}$ scale using alkylpyridinium salt $\mathbf{1 a}$ and enantiopure sulfinimine $\mathbf{2 b}$. The crude mixture was purified by silica gel chromatography $(20 \% \rightarrow 40 \%$ EtOAc/hexanes) to give $(S, S)-15(96 \mathrm{mg}, 54 \%,>20: 1 \mathrm{dr})$ as a white solid that had analytical data consistent with that noted above. The enantiomeric excess was determined to be $>99 \%$ by chiral SFC analysis (CHIRALPAK IA-3, $2.5 \mathrm{~mL} / \mathrm{min}, 5 \% \rightarrow 15 \%$ $\mathrm{MeOH}$ in $\left.\mathrm{CO}_{2}, \lambda=240 \mathrm{~nm}\right) ; t_{\mathrm{R}}($ major $)=4.4284 \mathrm{~min}, t_{\mathrm{R}}($ minor $)=4.0718 \mathrm{~min} .[\alpha]_{D}^{22}=+54.4(\mathrm{c}=$ $\left.1.29, \mathrm{CHCl}_{3}\right)$. 


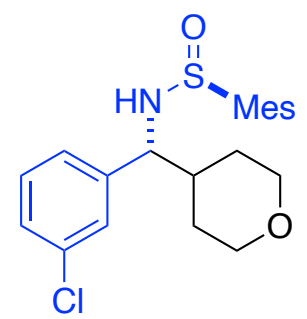

\section{2,4,6-Trimethyl- $N$-[2-methyl-1-(3-chlorophenyl)tetrahydro-2 $H$-pyran-4-}

yl)]benzenesulfinamide (16). Prepared via General Procedure A using alkylpyridinium salt 1a and sulfinimine 2c. The crude mixture was purified by silica gel chromatography $(1 \% \rightarrow 10 \%$ $\mathrm{MeOH} / \mathrm{DCM})$ to give $16\left(227 \mathrm{mg}, 58 \%,>20: 1 \mathrm{dr}\right.$ ) as a yellow solid (mp: $\left.150-154{ }^{\circ} \mathrm{C}\right):{ }^{1} \mathrm{H}$ NMR $\left(600 \mathrm{MHz}, \mathrm{CDCl}_{3}\right) \delta 7.23-7.20(\mathrm{~m}, 2 \mathrm{H}), 7.10(\mathrm{~d}, J=2.1 \mathrm{~Hz}, 1 \mathrm{H}), 7.09-7.06(\mathrm{~m}, 1 \mathrm{H}), 6.80(\mathrm{~s}$, 2H), $4.44(\mathrm{~d}, J=6.3 \mathrm{~Hz}, 1 \mathrm{H}), 4.15$ (dd, $J=7.7,6.2 \mathrm{~Hz}, 1 \mathrm{H}), 4.00$ (ddd, $J=11.7,4.7,1.8 \mathrm{~Hz}, 1 \mathrm{H}$ ), $3.90(\mathrm{ddd}, J=11.7,4.7,1.9 \mathrm{~Hz}, 1 \mathrm{H}), 3.35(\mathrm{td}, J=11.9,2.2 \mathrm{~Hz}, 1 \mathrm{H}), 3.29(\mathrm{td}, J=11.7,2.6 \mathrm{~Hz}$, $1 \mathrm{H}), 2.49$ (s, 6H), 2.26 (s, 3H), 1.98 (tdt, $J=11.6,7.6,3.8 \mathrm{~Hz}, 1 \mathrm{H}), 1.84$ (ddq, $J=13.2,4.3,2.2$ $\mathrm{Hz}, 1 \mathrm{H}), 1.37$ (dtd, $J=13.3,12.0,4.6 \mathrm{~Hz}, 1 \mathrm{H}), 1.31$ (ddq, $J=10.8,4.5,2.3 \mathrm{~Hz}, 1 \mathrm{H}), 1.25$ (dtd, $J$ $=13.3,11.7,4.5 \mathrm{~Hz}, 1 \mathrm{H}) ;{ }^{13} \mathrm{C} \mathrm{NMR}\left(151 \mathrm{MHz}, \mathrm{CDCl}_{3}\right) \delta 143.0,141.0,137.7,136.7,134.6,131.1$, 130.0, 128.0, 127.5, 125.6, 68.0, 67.7, 63.8, 40.9, 30.2, 29.7, 21.1, 19.6; FTIR (thin film) 3215, 2951, 2843, 2361, 1597, 1444, 1091, 1076, $698 \mathrm{~cm}^{-1}$; HRMS (ESI+) $[\mathrm{M}+\mathrm{H}]^{+}$calculated for $\mathrm{C}_{21} \mathrm{H}_{27} \mathrm{ClNO}_{2} \mathrm{~S}: 392.1451$, found 392.1433 .

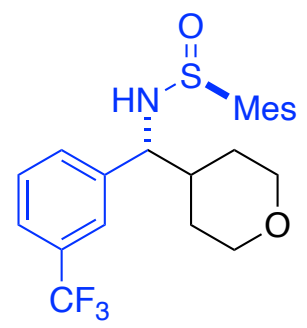

\section{2,4,6-Trimethyl- $N$-[2-methyl-1-(3-trifluoromethylphenyl)tetrahydro-2H-pyran-4-}

yl)]benzenesulfinamide (17). Prepared via General Procedure A using alkylpyridinium salt 1a and sulfinimine $2 \mathbf{d}$. The crude mixture was purified by silica gel chromatography $(20 \% \rightarrow 40 \%$ EtOAc/hexanes) to give 17 (285 mg, 67\%, >20:1 dr) as a white solid (mp: $\left.116-119{ }^{\circ} \mathrm{C}\right):{ }^{1} \mathrm{H}$ NMR $\left(400 \mathrm{MHz}, \mathrm{CDCl}_{3}\right) \delta 7.48(\mathrm{~d}, J=7.4 \mathrm{~Hz}, 1 \mathrm{H}), 7.42-7.31(\mathrm{~m}, 3 \mathrm{H}), 6.75(\mathrm{~s}, 2 \mathrm{H}), 4.52(\mathrm{~d}, J=6.1$ $\mathrm{Hz}, 1 \mathrm{H}), 4.26(\mathrm{t}, J=6.8 \mathrm{~Hz}, 1 \mathrm{H}), 4.00(\mathrm{dd}, J=11.7,4.4 \mathrm{~Hz}, 1 \mathrm{H}), 3.96-3.88(\mathrm{~m}, 1 \mathrm{H}), 3.40-3.24$ (m, 2H), 2.47 (s, 6H), 2.23 (s, 3H), 1.99 (dddd, $J=15.7,11.5,8.2,4.4 \mathrm{~Hz}, 1 \mathrm{H}), 1.84$ (d, $J=13.2$ $\mathrm{Hz}, 1 \mathrm{H}), 1.45-1.26(\mathrm{~m}, 3 \mathrm{H}) ;{ }^{13} \mathrm{C} \mathrm{NMR}\left(101 \mathrm{MHz}, \mathrm{CDCl}_{3}\right) \delta 141.7,140.9,137.0,136.6,130.9$, $130.7\left(\mathrm{q}, J_{\mathrm{C}-\mathrm{F}}=32.3 \mathrm{~Hz}\right), 130.2,128.9,124.4\left(\mathrm{q}, J_{\mathrm{C}-\mathrm{F}}=4.0 \mathrm{~Hz}\right), 124.0\left(\mathrm{q}, J_{\mathrm{C}-\mathrm{F}}=272.7 \mathrm{~Hz}\right), 123.7$ 
$\left(\mathrm{q}, J_{\mathrm{C}-\mathrm{F}}=3.3 \mathrm{~Hz}\right), 67.8,67.5,63.2,40.8,29.9,29.5,20.9,19.4 ;{ }^{19} \mathrm{~F} \mathrm{NMR}\left(376 \mathrm{MHz}, \mathrm{CDCl}_{3}\right) \delta-$ 62.62; FTIR (thin film) 3236, 2917, 2848, 2361, 2336, 1328, 1163, 1124, 1074, 850, $667 \mathrm{~cm}^{-1}$; HRMS (ESI + ) $[\mathrm{M}+\mathrm{H}]^{+}$calculated for $\mathrm{C}_{22} \mathrm{H}_{27} \mathrm{~F}_{3} \mathrm{NO}_{2} \mathrm{~S}: 426.1715$, found 426.1701 .<smiles>CS(=O)NC(c1cccc(C(F)(F)F)c1)C1CCOCC1</smiles>

\section{(S)-2,4,6-trimethyl- $N$-[(S)-2-methyl-1-(3-trifluoromethylphenyl)tetrahydro-2H-pyran-4-}

yl)]benzenesulfinamide $((S, S)-17)$. Prepared via General Procedure A on a $0.5 \mathrm{mmol}$ scale using alkylpyridinium salt 1a and enantiopure sulfinimine $\mathbf{2 d}$. The crude mixture was purified by silica gel chromatography $(20 \% \rightarrow 40 \%$ EtOAc/hexanes $)$ to give $(\boldsymbol{S}, \boldsymbol{S})-17(140 \mathrm{mg}, 66 \%,>20: 1 \mathrm{dr})$ as a white solid that had analytical data consistent with that noted above. The enantiomeric excess was determined to be $>99 \%$ by chiral SFC analysis (CHIRALPAK ID-3, $2.5 \mathrm{~mL} / \mathrm{min}, 5 \% \rightarrow 15 \%$ $\mathrm{MeOH}$ in $\left.\left.\mathrm{CO}_{2}, \lambda=254 \mathrm{~nm}\right) ;\right)$; $t_{\mathrm{R}}($ major $)=3.3101 \mathrm{~min}, t_{\mathrm{R}}($ minor $)=2.8417 \mathrm{~min} .[\alpha]_{D}^{22}=+0.4(\mathrm{c}=$ $\left.1.03, \mathrm{CHCl}_{3}\right)$.

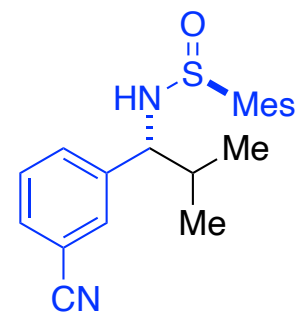

2,4,6-Trimethyl- $N$-[2-methyl-1-(3-cyanophenyl)propyl]benzenesulfinamide (18). Prepared via General Procedure A using alkylpyridinium salt 1g and sulfinimine 2e. The crude mixture was purified by silica gel chromatography $(1 \% \rightarrow 10 \% \mathrm{MeOH} / \mathrm{DCM})$ to give $18(286 \mathrm{mg}, 84 \%,>20: 1$ dr) as a red-brown solid (mp: $\left.98-100{ }^{\circ} \mathrm{C}\right):{ }^{1} \mathrm{H}$ NMR $\left(600 \mathrm{MHz}, \mathrm{CDCl}_{3}\right) \delta 7.49(\mathrm{dt}, J=7.6,1.4$ $\mathrm{Hz}, 1 \mathrm{H}), 7.44(\mathrm{dt}, J=8.0,1.6 \mathrm{~Hz}, 1 \mathrm{H}), 7.39-7.34(\mathrm{~m}, 2 \mathrm{H}), 6.75(\mathrm{~s}, 2 \mathrm{H}), 4.44(\mathrm{~d}, J=5.5 \mathrm{~Hz}, 1 \mathrm{H})$, $4.27(\mathrm{t}, J=6.0 \mathrm{~Hz}, 1 \mathrm{H}), 2.49$ (s, 6H), $2.24(\mathrm{~s}, 3 \mathrm{H}), 2.13$ (h, $J=6.7 \mathrm{~Hz}, 1 \mathrm{H}), 0.96$ (d, $J=6.7 \mathrm{~Hz}$, $3 \mathrm{H}), 0.82(\mathrm{~d}, J=6.7 \mathrm{~Hz}, 3 \mathrm{H}) ;{ }^{13} \mathrm{C} \mathrm{NMR}\left(151 \mathrm{MHz}, \mathrm{CDCl}_{3}\right) \delta 142.9,141.0,137.0,136.8,132.0$, 131.1, 131.0, 130.9, 129.0, 118.8, 112.4, 63.5, 33.6, 21.1, 19.6, 19.2, 18.8; FTIR (thin film) 3207, 2962, 2928, 2228, 1251, 1071, 1048, 849, $696 \mathrm{~cm}^{-1}$; HRMS (ESI+) $[\mathrm{M}+\mathrm{H}]^{+}$calculated for $\mathrm{C}_{20} \mathrm{H}_{25} \mathrm{~N}_{2} \mathrm{OS}$ : 341.1688 , found 341.1676. 


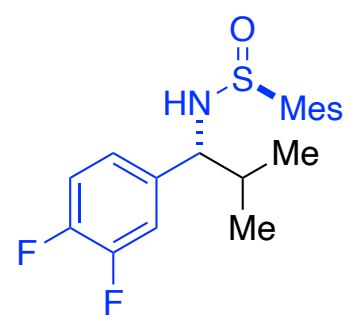

2,4,6-Trimethyl- $N$-[2-methyl-1-(3,4-difluorophenyl)propyl]benzenesulfinamide

(19).

Prepared via General Procedure A using alkylpyridinium salt $\mathbf{1 g}$ and sulfinimine $\mathbf{2 f}$. The crude mixture was purified by silica gel chromatography $(1 \% \rightarrow 10 \% \mathrm{MeOH} / \mathrm{DCM})$ to give $19(323 \mathrm{mg}$, 92\%, >20:1 dr) as a light yellow solid (mp: $\left.89-91{ }^{\circ} \mathrm{C}\right):{ }^{1} \mathrm{H}$ NMR $\left(600 \mathrm{MHz}, \mathrm{CDCl}_{3}\right) \delta 7.06(\mathrm{dt}, J$ $=10.1,8.3 \mathrm{~Hz}, 1 \mathrm{H}), 7.00(\mathrm{ddd}, J=11.4,7.6,2.2 \mathrm{~Hz}, 1 \mathrm{H}), 6.94(\mathrm{ddt}, J=8.0,3.8,1.7 \mathrm{~Hz}, 1 \mathrm{H}), 6.80$ (s, 2H), 4.39 (d, $J=5.6 \mathrm{~Hz}, 1 \mathrm{H}), 4.18(\mathrm{t}, J=6.1 \mathrm{~Hz}, 1 \mathrm{H}), 2.51(\mathrm{~s}, 6 \mathrm{H}), 2.26(\mathrm{~s}, 3 \mathrm{H}), 2.13(\mathrm{~h}, J=$ $6.7 \mathrm{~Hz}, 1 \mathrm{H}), 0.95(\mathrm{~d}, J=6.7 \mathrm{~Hz}, 3 \mathrm{H}), 0.82(\mathrm{~d}, J=6.7 \mathrm{~Hz}, 3 \mathrm{H}) ;{ }^{13} \mathrm{C} \mathrm{NMR}\left(151 \mathrm{MHz}, \mathrm{CDCl}_{3}\right) \delta$ $150.3\left(\mathrm{dd}, J_{\mathrm{C}-\mathrm{F}}=249.15,13.6 \mathrm{~Hz}\right), 149.8\left(\mathrm{dd}, J_{\mathrm{C}-\mathrm{F}}=247.64,12.1 \mathrm{~Hz}\right), 141.0,138.5\left(\mathrm{app} \mathrm{t}, J_{\mathrm{C}-\mathrm{F}}=\right.$ $4.5 \mathrm{~Hz}), 137.9,136.8,131.1,123.7\left(\mathrm{dd}, J_{\mathrm{C}-\mathrm{F}}=6.0,3.0 \mathrm{~Hz}\right), 117.2\left(\mathrm{~d}, J_{\mathrm{C}-\mathrm{F}}=18.1 \mathrm{~Hz}\right), 116.5\left(\mathrm{~d}, J_{\mathrm{C}-}\right.$ $\mathrm{F}=16.6 \mathrm{~Hz}), 64.0,33.5,21.2,19.7,19.4,18.8 ;{ }^{19} \mathrm{~F} \mathrm{NMR}\left(565 \mathrm{MHz}, \mathrm{CDCl}_{3}\right) \delta-137.3(\mathrm{~d}, J=21.2$ $\mathrm{Hz}$ ), -139.4 (d, $J=21.2 \mathrm{~Hz}$ ); FTIR (thin film) 2982, 1517, 1436, 1277, $1071 \mathrm{~cm}^{-1}$; HRMS (ESI+) $[\mathrm{M}+\mathrm{H}]^{+}$calculated for $\mathrm{C}_{19} \mathrm{H}_{24} \mathrm{~F}_{2} \mathrm{NOS}$ : 352.1547 , found 352.1537 .

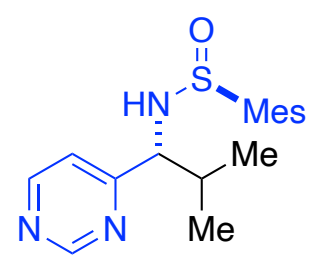

2,4,6-Trimethyl- $N$-[2-methyl-1-(4-pyrimidinyl)propyl]benzenesulfinamide (20). Prepared via General Procedure A on a $0.25 \mathrm{mmol}$ scale using alkylpyridinium salt $\mathbf{1 g}$ and sulfinimine $\mathbf{2 g}$. The crude mixture was purified by silica gel chromatography $(1 \% \rightarrow 10 \% \mathrm{MeOH} / \mathrm{DCM})$ to give $\mathbf{2 0}$ $(62 \mathrm{mg}, 83 \%,>20: 1 \mathrm{dr})$ as a red solid $\left(\mathrm{mp}: 132-135{ }^{\circ} \mathrm{C}\right):{ }^{1} \mathrm{H}$ NMR $\left(600 \mathrm{MHz}, \mathrm{CDCl}_{3}\right) \delta 9.16(\mathrm{~d}$, $J=1.3 \mathrm{~Hz}, 1 \mathrm{H}), 8.64(\mathrm{~d}, J=5.0 \mathrm{~Hz}, 1 \mathrm{H}), 7.23(\mathrm{dd}, J=5.2,1.4 \mathrm{~Hz}, 1 \mathrm{H}), 6.85(\mathrm{~s}, 2 \mathrm{H}), 5.84(\mathrm{~d}, J=$ $8.5 \mathrm{~Hz}, 1 \mathrm{H}), 4.20$ (dd, $J=8.5,6.0 \mathrm{~Hz}, 1 \mathrm{H}), 2.56$ (s, 6H), 2.28 (d, $J=2.6 \mathrm{~Hz}, 3 \mathrm{H}), 2.03$ (h, $J=6.6$ $\mathrm{Hz}, 1 \mathrm{H}), 0.92(\mathrm{~d}, J=6.7 \mathrm{~Hz}, 3 \mathrm{H}), 0.85(\mathrm{~d}, J=6.7 \mathrm{~Hz}, 3 \mathrm{H}) ;{ }^{13} \mathrm{C} \mathrm{NMR}\left(151 \mathrm{MHz}, \mathrm{CDCl}_{3}\right) \delta 169.4$, 158.4, 157.0, 140.9, 138.5, 136.7, 130.9, 120.0, 65.0, 34.8, 21.2, 19.9, 19.6, 17.7; FTIR (thin film) 3222, 2962, 2928, 1580, 1384, 1084, 848, $699 \mathrm{~cm}^{-1}$; HRMS (ESI +$)[\mathrm{M}+\mathrm{H}]^{+}$calculated for $\mathrm{C}_{17} \mathrm{H}_{24} \mathrm{~N}_{3} \mathrm{OS}: 318.1640$, found 318.1629 . 


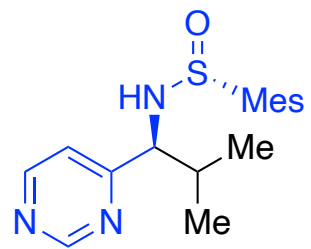

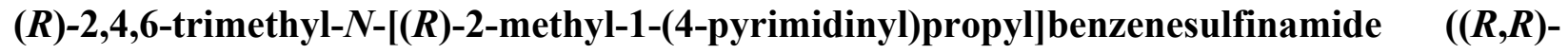
20). Prepared via General Procedure A using alkylpyridinium salt 1g and enantiopure sulfinimine 2g. The crude mixture was purified by silica gel chromatography $(1 \% \rightarrow 10 \% \mathrm{MeOH} / \mathrm{DCM})$ to give $(\boldsymbol{R}, \boldsymbol{R})-20(279 \mathrm{mg}, 88 \%,>20: 1 \mathrm{dr})$ as a white solid that had analytical data consistent with that noted above. The enantiomeric excess was determined to be $>99 \%$ by chiral SFC analysis $\left(\right.$ CHIRALPAK ID-3, $2.5 \mathrm{~mL} / \mathrm{min}, 5 \% \rightarrow 15 \% \mathrm{MeOH}$ in $\mathrm{CO}_{2}, \lambda=230 \mathrm{~nm}$ ); $t_{\mathrm{R}}$ (major) $=3.5535 \mathrm{~min}$, $t_{\mathrm{R}}($ minor $)=4.2585 \min .[\alpha]_{D}^{22}=-133.7\left(\mathrm{c}=0.53, \mathrm{CHCl}_{3}\right)$.

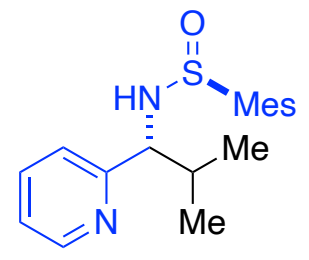

2,4,6-Trimethyl- $N$-[2-methyl-1-(2-pyridinyl)propyl]benzenesulfinamide (21). Prepared via General Procedure A using alkylpyridinium salt $\mathbf{1 g}$ and sulfinimine $\mathbf{2 h}$ on a $0.5 \mathrm{mmol}$ scale. The crude mixture was purified by silica gel chromatography $(1 \% \rightarrow 10 \% \mathrm{MeOH} / \mathrm{DCM})$ to give 21 (134 $\mathrm{mg}, 85 \%,>20: 1 \mathrm{dr}$ ) as a dark red/brown oil that had analytical data consistent with the literature ${ }^{2}:{ }^{1} \mathrm{H} \mathrm{NMR}\left(600 \mathrm{MHz} \mathrm{CDCl}_{3}\right) \delta 8.54(\mathrm{ddd}, J=4.9,1.8,0.9 \mathrm{~Hz}, 1 \mathrm{H}), 7.62(\mathrm{td}, J=7.6$, $1.8 \mathrm{~Hz}, 1 \mathrm{H}), 7.20(\mathrm{dt}, J=7.8,1.1 \mathrm{~Hz}, 1 \mathrm{H}), 7.16(\mathrm{ddd}, J=7.5,4.8,1.1 \mathrm{~Hz}, 1 \mathrm{H}), 6.85(\mathrm{~s}, 2 \mathrm{H}), 6.07$ $(\mathrm{d}, J=7.9 \mathrm{~Hz}, 1 \mathrm{H}), 4.24(\mathrm{dd}, J=7.9,6.1 \mathrm{~Hz}, 1 \mathrm{H}), 2.57$ (s, 6H), 2.28 (s, 3H), $2.02(\mathrm{~h}, J=6.7 \mathrm{~Hz}$, $1 \mathrm{H}), 0.90(\mathrm{~d}, J=6.7 \mathrm{~Hz}, 3 \mathrm{H}), 0.85(\mathrm{~d}, J=6.7 \mathrm{~Hz}, 3 \mathrm{H}) ;{ }^{13} \mathrm{C} \mathrm{NMR}\left(151 \mathrm{MHz}, \mathrm{CDCl}_{3}\right) \delta 160.7$, $148.7,140.3,139.0,136.5,136.3,130.1,122.4,122.2,65.3,251,21.0,19.8,17.7$.

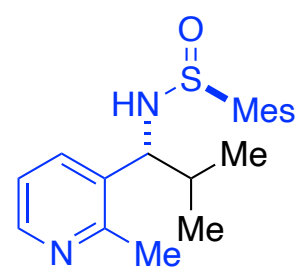

\section{2,4,6-Trimethyl- $N$-[2-methyl-1-(2-methyl-3-pyridinyl)propyl]benzenesulfinamide}

(22).

Prepared via General Procedure A using alkylpyridinium salt $1 \mathbf{g}$ and sulfinimine 2i. The crude mixture was purified by silica gel chromatography $(20 \% \rightarrow 50 \%$ EtOAc/hexanes) to give 22 (178 
$\mathrm{mg}, 54 \%,>20: 1 \mathrm{dr}$ ) as a yellow solid (mp: $\left.98-100{ }^{\circ} \mathrm{C}\right):{ }^{1} \mathrm{H} \mathrm{NMR}\left(600 \mathrm{MHz}, \mathrm{CDCl}_{3}\right) \delta 8.39$ (dd, $J=4.8,1.7 \mathrm{~Hz}, 1 \mathrm{H}), 7.55(\mathrm{~d}, J=7.7 \mathrm{~Hz}, 1 \mathrm{H}), 7.11(\mathrm{dd}, J=7.9,4.7 \mathrm{~Hz}, 1 \mathrm{H}), 6.80-6.78(\mathrm{~m}, 2 \mathrm{H})$, $4.46(\mathrm{~d}, J=6.3 \mathrm{~Hz}, 1 \mathrm{H}), 4.38$ (t, $J=6.7 \mathrm{~Hz}, 1 \mathrm{H}), 2.59$ (d, $J=2.1 \mathrm{~Hz}, 3 \mathrm{H}), 2.48$ (s, 6H), 2.13 (dq, $J=13.6,6.8 \mathrm{~Hz}, 1 \mathrm{H}), 1.02(\mathrm{~d}, J=6.7 \mathrm{~Hz}, 3 \mathrm{H}), 0.87(\mathrm{~d}, J=6.8 \mathrm{~Hz}, 3 \mathrm{H}) ;{ }^{13} \mathrm{C} \mathrm{NMR}(101 \mathrm{MHz}$, $\left.\mathrm{CDCl}_{3}\right) \delta 155.9,147.7,140.8,137.9,136.6,135.6,134.4,131.0,60.8,33.9,22.5,21.1,19.6,19.5$, 18.9; FTIR (thin film) 3218, 2959, 2927, 2870. 1443. 1072, 1047, 849, $738 \mathrm{~cm}^{-1}$; HRMS (ESI+) $[\mathrm{M}+\mathrm{H}]^{+}$calculated for $\mathrm{C}_{19} \mathrm{H}_{27} \mathrm{~N}_{2} \mathrm{OS}: 331.1844$, found 331.1832 .

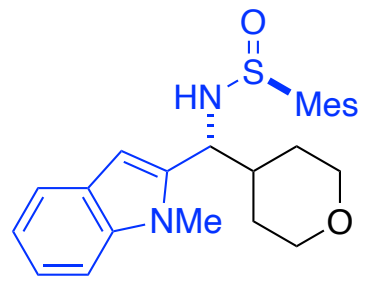

\section{2,4,6-Trimethyl- $N$-[2-methyl-1-(1-methyl-1H-indol-2-yl)tetrahydro-2H-pyran-4-}

yl)]benzenesulfinamide (23). Prepared via General Procedure A using alkylpyridinium salt 1a and sulfinimine $\mathbf{2 \mathbf { j }}$. The crude mixture was purified by silica gel chromatography $(20 \% \rightarrow 60 \%$ EtOAc/hexanes) to give 23 (186 mg, 46\%, >20:1 dr) as a dark orange solid (mp: $\left.118-120{ }^{\circ} \mathrm{C}\right)$ : ${ }^{1} \mathrm{H} \mathrm{NMR}\left(400 \mathrm{MHz}, \mathrm{CDCl}_{3}\right) \delta 7.62-7.51(\mathrm{~m}, 1 \mathrm{H}), 7.40-7.32(\mathrm{~m}, 1 \mathrm{H}), 7.25-7.20(\mathrm{~m}, 1 \mathrm{H}), 7.11$ (ddd, $J=8.0,7.0,1.0 \mathrm{~Hz}, 1 \mathrm{H}), 6.83(\mathrm{~s}, 2 \mathrm{H}), 6.43(\mathrm{~s}, 1 \mathrm{H}), 4.51$ (d, $J=5.7 \mathrm{~Hz}, 1 \mathrm{H}), 4.38$ (dd, $J=$ 8.8, $5.7 \mathrm{~Hz}, 1 \mathrm{H}), 4.09-4.02(\mathrm{~m}, 1 \mathrm{H}), 3.96-3.88(\mathrm{~m}, 1 \mathrm{H}), 3.82(\mathrm{~s}, 3 \mathrm{H}), 3.38(\mathrm{dtd}, J=23.3,11.9$, $2.2 \mathrm{~Hz}, 2 \mathrm{H}), 2.46(\mathrm{~s}, 6 \mathrm{H}), 2.26(\mathrm{~s}, 3 \mathrm{H}), 2.23-2.15(\mathrm{~m}, 1 \mathrm{H}), 2.09-2.01(\mathrm{~m}, 1 \mathrm{H}), 1.53-1.23(\mathrm{~m}$, $3 \mathrm{H}) ;{ }^{13} \mathrm{C}$ NMR $\left(101 \mathrm{MHz}, \mathrm{CDCl}_{3}\right) \delta 141 ., 139.5,138.4,137.4,136.4,131.0,127.5,121.9,120.6$, 119.9, 109.7, 99.8, 67.9, 67.7, 56.7, 41.2, 30.5, 30.4, 30.2, 21.2, 19.5; FTIR (thin film) 2921, 1349, 1083, 1048, $615 \mathrm{~cm}^{-1}$; HRMS (ESI+) $[\mathrm{M}+\mathrm{H}]^{+}$calculated for $\mathrm{C}_{24} \mathrm{H}_{31} \mathrm{~N}_{2} \mathrm{O}_{2} \mathrm{~S}: 411.2106$, found 411.2091.

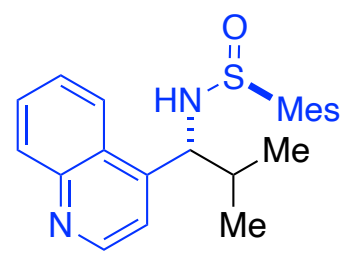

2,4,6-Trimethyl- $N$-[2-methyl-1-(4-quinolinyl)propyl]benzenesulfinamide (24). Prepared via General Procedure A using alkylpyridinium salt 1g and sulfinimine 2k. The crude mixture was purified by silica gel chromatography $(30 \% \rightarrow 60 \%$ EtOAc/hexanes) to give 24 (185 mg, 51\%, $>20: 1 \mathrm{dr})$ as a dark red/brown solid (mp: $\left.150-152{ }^{\circ} \mathrm{C}\right):{ }^{1} \mathrm{H} \mathrm{NMR}\left(400 \mathrm{MHz}, \mathrm{CDCl}_{3}\right) \delta 8.81(\mathrm{~d}, J$ 
$=4.6 \mathrm{~Hz}, 1 \mathrm{H}), 8.14(\mathrm{~d}, J=8.2 \mathrm{~Hz}, 1 \mathrm{H}), 8.04(\mathrm{~d}, J=8.5 \mathrm{~Hz}, 1 \mathrm{H}), 7.73(\mathrm{t}, J=7.6 \mathrm{~Hz}, 1 \mathrm{H}), 7.58(\mathrm{t}$, $J=7.7 \mathrm{~Hz}, 1 \mathrm{H}), 7.33(\mathrm{~d}, J=4.6 \mathrm{~Hz}, 1 \mathrm{H}), 6.66(\mathrm{~s}, 2 \mathrm{H}), 5.08(\mathrm{t}, J=6.2 \mathrm{~Hz}, 1 \mathrm{H}), 4.71(\mathrm{~d}, J=6.3$ $\mathrm{Hz}, 1 \mathrm{H}), 2.46$ (s, 6H), $2.31(\mathrm{dd}, J=14.4,7.3 \mathrm{~Hz}, 1 \mathrm{H}), 2.17(\mathrm{~s}, 3 \mathrm{H}), 0.97(\mathrm{~d}, J=6.7 \mathrm{~Hz}, 6 \mathrm{H}) ;{ }^{13} \mathrm{C}$ NMR $\left(101 \mathrm{MHz}, \mathrm{CDCl}_{3}\right) \delta 149.7,148.4,147.9,140.8,137.3,136.7,130.9,130.5,129.3,126.7$, 126.2, 123.1, 119.0, 59.4, 33.9, 21.0, 20.1, 19.6, 18.3; FTIR (thin film) 2965, 1240, 1072, 849, $757,621 \mathrm{~cm}^{-1}$; HRMS (ESI+) $[\mathrm{M}+\mathrm{H}]^{+}$calculated for $\mathrm{C}_{22} \mathrm{H}_{27} \mathrm{~N}_{2} \mathrm{OS}: 367.1844$, found 367.1829 .

\section{Preparation of Sulfinimines}

\section{Synthesis of 2,4,6-trimethyl-benzeneulfinamide}<smiles>Cc1cc(C)c(S(=O)(=O)Cl)c(C)c1</smiles>
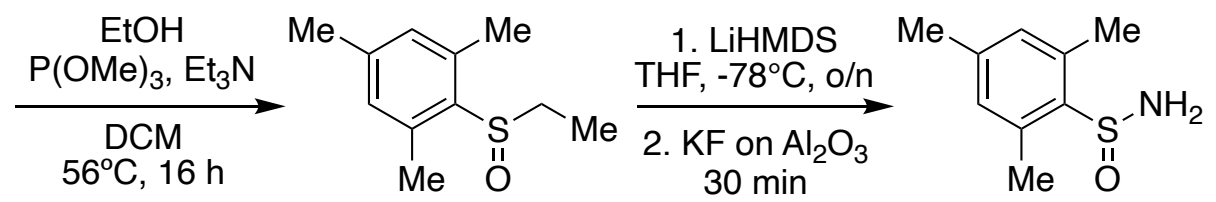

This procedure is adapted from that reported in the literature. ${ }^{3}$ In our hands, freshly dissolving solid LiHMDS in THF and changing the order of addition of the second step was more effective. An oven-dried, 250-mL round-bottomed flask was charged with 2,4,6trimethylbenzenesulfonyl chloride ( $4.4 \mathrm{~g}, 20 \mathrm{mmol}, 1.0$ equiv) and a stir bar and fitted with a septum with a $\mathrm{N}_{2}$ inlet. Anhydrous $\mathrm{CH}_{2} \mathrm{Cl}_{2}(90 \mathrm{~mL})$ was added via syringe, followed by EtOH (1.0 $\mathrm{mL}, 18 \mathrm{mmol}, 0.9$ equiv), $\mathrm{Et}_{3} \mathrm{~N}$ (3.6 mL, $26 \mathrm{mmol}, 1.3$ equiv), and trimethyl phosphite (4.4 mL, $35 \mathrm{mmol}, 1.8$ equiv). The flask was fitted with a reflux condenser and refluxed with stirring for 16 $\mathrm{h}$ in an oil bath set at $56^{\circ} \mathrm{C}$. The mixture was cooled and added to a separatory funnel containing $\mathrm{Et}_{2} \mathrm{O}(100 \mathrm{~mL})$, EtOAc $(100 \mathrm{~mL})$, and $1 \mathrm{~N} \mathrm{HCl}(100 \mathrm{~mL})$. The layers were separated. Then the organic layer was washed with saturated aqueous $\mathrm{NaHCO}_{3}(3 \times 100 \mathrm{~mL})$ and 1:1 $\mathrm{H}_{2} \mathrm{O}$ :brine $(3 \mathrm{x}$ $100 \mathrm{~mL}$ ), dried with $\mathrm{MgSO}_{4}$, filtered, and concentrated. The crude material is unstable and was used immediately in the next step.

In a $\mathrm{N}_{2}$-atmosphere glovebox, solid LiHMDS (5.5 g, $\left.33 \mathrm{mmol}\right)$ was added to an oven-dried, 250-mL round-bottomed flask charged with a stir bar. The flask was fitted with a septum and removed from the glovebox, and a $\mathrm{N}_{2}$ inlet was added. Anhydrous THF $(50 \mathrm{~mL})$ was added to the flask via syringe, and the mixture was stirred for 5 minutes at room temperature or until the solid was dissolved. The solution was cooled to $-78^{\circ} \mathrm{C}$. The crude material from the first step was added via cannula, rinsing the original flask with anhydrous THF $(50 \mathrm{~mL})$. The solution was stirred and allowed to warm to room temperature overnight. Potassium fluoride on alumina (1.6 g) was added, 
and the mixture was stirred for 30 minutes at room temperature. The mixture was then added to a separatory funnel containing $\mathrm{Et}_{2} \mathrm{O}(125 \mathrm{~mL})$, EtOAc $(75 \mathrm{~mL})$, and saturated aqueous $\mathrm{NaHCO}_{3}(50$ $\mathrm{mL}$ ). The layers were separated. Then the organic layer was washed with saturated aqueous $\mathrm{NaHCO}_{3}(3 \times 100 \mathrm{~mL})$ and brine $(3 \times 100 \mathrm{~mL})$, dried with $\mathrm{MgSO}_{4}$, filtered, and concentrated. The crude material was then purified by silica gel chromatography $(50 \% \rightarrow 75 \%$ EtOAc/hexanes $)$ to give 2,4,6-trimethylbenzenesulfinamide (1.46 g, 40\%) as a white solid that had analytical data consistent with the literature ${ }^{3}:{ }^{1} \mathrm{H}$ NMR $\left(600 \mathrm{MHz} \mathrm{CDCl}_{3}\right) \delta 6.86(\mathrm{~s}, 2 \mathrm{H}), 4.39(\mathrm{~s}, 2 \mathrm{H}), 2.60(\mathrm{~s}$, 6H), 2.28 (s, 3H); ${ }^{13} \mathrm{C}$ NMR (151 MHz, $\left.\mathrm{CDCl}_{3}\right) \delta 140.9,139.1,136.4,131.0,21.1,19.4$.

\section{General Procedure B: Synthesis of Sulfinimines}

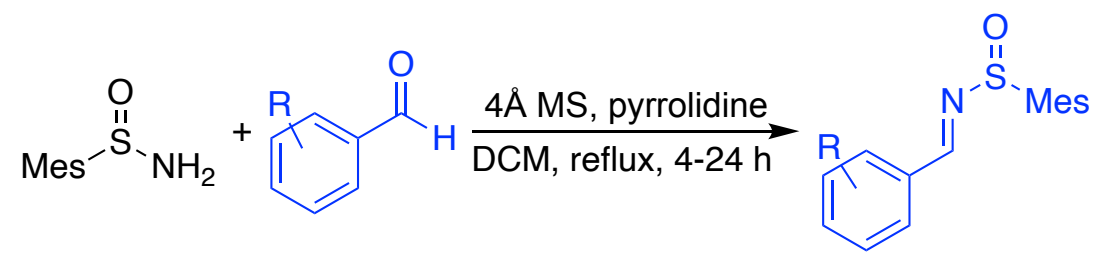

This procedure was adapted from that reported in the literature. ${ }^{4}$ Under air, 2,4,6trimethylbenzenesulfinamide (1.0 equiv), powered activated $4 \AA$ molecular sieves ( $1 \mathrm{~g} / \mathrm{mmol})$, and $\mathrm{CH}_{2} \mathrm{Cl}_{2}(0.32 \mathrm{M})$ were added to a round-bottomed flask with a stir bar. The aldehyde (1.0 equiv) was added, followed by pyrrolidine ( 0.10 equiv), and the flask was fitted with a reflux condenser. The mixture was refluxed for $4-24 \mathrm{~h}$ at $55^{\circ} \mathrm{C}$ in an oil bath. The mixture was filtered through a short Celite ${ }^{\circledR}$ plug rinsing with $\mathrm{CH}_{2} \mathrm{Cl}_{2}$, and the filtrate was concentrated. The solid sulfinimine was purified by silica gel chromatography if needed.

The following known sulfinimines were prepared according to this procedure and had analytical data consistent with that reported in the literature: $\mathbf{2 a}, \mathbf{2 c}$, and $\mathbf{2} \mathbf{i} ;{ }^{5} \mathbf{2} \mathbf{b}$ and $\mathbf{2 h} .^{2}$ 
<smiles>CS(=O)/N=C/c1ccccc1</smiles>

2b<smiles>CS(=O)/N=C/c1ccc(F)c(F)c1</smiles><smiles>CS(=O)/N=C/c1cccc(Cl)c1</smiles>

2c<smiles>CS(=O)N=Cc1cccnc1</smiles><smiles>CS(=O)/N=C/c1cccc(C(F)(F)F)c1</smiles>

2d<smiles>CS(=O)/N=C/c1ccncn1</smiles>

2g<smiles>CS(=O)/N=C/c1cccc(C#N)c1</smiles>

$2 e$<smiles>Cc1ncccc1/C=N/S(C)=O</smiles>

2i

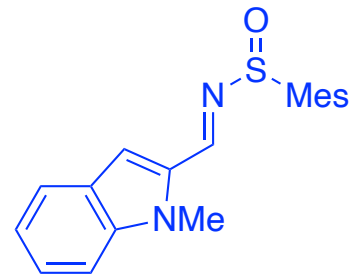

2j<smiles>CS(=O)/N=C/c1ccnc2ccccc12</smiles>

2k<smiles>CS(=O)/N=C/c1ccccn1</smiles>

$2 \mathrm{~h}$<smiles>CS(=O)/N=C/c1cccc(C(F)(F)F)c1</smiles>

2,4,6-Trimethyl- $N$-(3-trifluoromethylbenzylidene)benzenesulfinamide (2d). Prepared via General Procedure B from 3-(trifluoromethyl)benzaldehyde (0.14 mL, $1.0 \mathrm{mmol}, 1.0$ equiv) and 2,4,6-trimethylbenzenesulfinamide (183 mg, $1.0 \mathrm{mmol}, 1.0$ equiv) to give $\mathbf{2 d}(244 \mathrm{mg}, 72 \%)$ as a light yellow solid (mp: $\left.102-104{ }^{\circ} \mathrm{C}\right):{ }^{1} \mathrm{H}$ NMR (600 MHz, $\left.\mathrm{CDCl}_{3}\right) \delta 8.87(\mathrm{~s}, 1 \mathrm{H}), 8.14(\mathrm{~s}, 1 \mathrm{H})$, $8.01(\mathrm{~d}, J=7.7 \mathrm{~Hz}, 1 \mathrm{H}), 7.76(\mathrm{~d}, J=7.8 \mathrm{~Hz}, 1 \mathrm{H}), 7.60(\mathrm{t}, J=7.8 \mathrm{~Hz}, 1 \mathrm{H}), 6.88(\mathrm{~s}, 2 \mathrm{H}), 2.50$ (s, 6H), $2.30(\mathrm{~s}, 3 \mathrm{H}) ;{ }^{13} \mathrm{C} \mathrm{NMR}\left(151 \mathrm{MHz}, \mathrm{CDCl}_{3}\right) \delta 160.3,142.1,138.7,134.7,133.0,131.8\left(\mathrm{q}, J_{\mathrm{C}-}\right.$ $\mathrm{F}=33.22 \mathrm{~Hz}), 131.1,129.7,128.9\left(\mathrm{q}, J_{\mathrm{C}-\mathrm{F}}=4.53 \mathrm{~Hz}\right), 126.0\left(\mathrm{q}, J_{\mathrm{C}-\mathrm{F}}=4.53 \mathrm{~Hz}\right) 123.8\left(\mathrm{q}, J_{\mathrm{C}-\mathrm{F}}=\right.$ $273.31 \mathrm{~Hz}), 21.3,19.0 ;{ }^{19} \mathrm{~F}$ NMR $\left(565 \mathrm{MHz}, \mathrm{CDCl}_{3}\right) \delta-62.86$; FTIR (thin film) 2953, 1328, 1202, 
1128, 1093, 1069, $695 \mathrm{~cm}^{-1}$; HRMS $\left(\mathrm{ESI}^{+}\right)[\mathrm{M}+\mathrm{H}]^{+}$calculated for $\mathrm{C}_{17} \mathrm{H}_{17} \mathrm{~F}_{3} \mathrm{NOS}: 340.0983$, found 340.0973 .

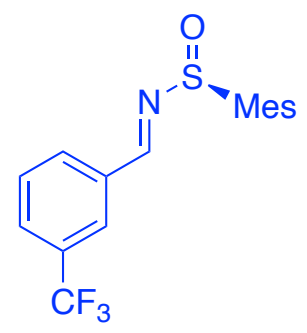

(S)-2,4,6-Trimethyl- $N$-(3-trifluoromethylbenzylidene)benzenesulfinamide ((S)-2d). Prepared via General Procedure B from 3-(trifluoromethyl)benzaldehyde (0.14 mL, $1.0 \mathrm{mmol}, 1.0$ equiv) and $S$-2,4,6-trimethylbenzenesulfinamide (183 mg, $1.0 \mathrm{mmol}, 1.0$ equiv) to give $(\boldsymbol{S})$-2d (237 $\mathrm{mg}$, $70 \%$ ) as a light yellow solid that had analytical data consistent with that noted above. $[\alpha]_{D}^{22}=+95.2$ $\left(\mathrm{c}=1.67, \mathrm{CHCl}_{3}\right)$.

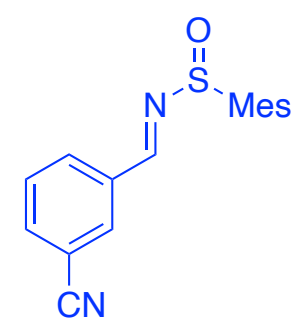

2,4,6-Trimethyl- $N$-(3-cyanobenzylidene)benzenesulfinamide (2e). Prepared via General Procedure B from 3-(cyano)benzaldehyde $(0.26 \mathrm{~mL}, 2.0 \mathrm{mmol}, 2.0$ equiv) and 2,4,6trimethylbenzenesulfinamide (366 mg, $2.0 \mathrm{mmol}, 2.0$ equiv) to give $2 \mathrm{e}$ ( $486 \mathrm{mg}, 82 \%$ ) as a white solid (mp: $\left.136-139{ }^{\circ} \mathrm{C}\right):{ }^{1} \mathrm{H}$ NMR $\left(400 \mathrm{MHz} \mathrm{CDCl}_{3}\right) \delta 8.82(\mathrm{~s}, 1 \mathrm{H}), 8.16(\mathrm{~d}, J=1.7 \mathrm{~Hz}, 1 \mathrm{H})$, $8.05(\mathrm{dt}, J=7.9,1.5 \mathrm{~Hz}, 1 \mathrm{H}), 7.78(\mathrm{dt}, J=7.8,1.5 \mathrm{~Hz}, 1 \mathrm{H}), 7.60(\mathrm{t}, J=7.8 \mathrm{~Hz}, 1 \mathrm{H}), 6.88(\mathrm{~s}, 2 \mathrm{H})$, $2.49(\mathrm{~s}, 6 \mathrm{H}), 2.30(\mathrm{~s}, 3 \mathrm{H}) ;{ }^{13} \mathrm{C} \mathrm{NMR}\left(101 \mathrm{MHz}, \mathrm{CDCl}_{3}\right) \delta 159.4,142.3,138.6,135.4,135.0,134.68$, 134.67, 133.7, 132.7, 131.2, 130.0, 118.0, 113.7, 21.3, 19.0; FTIR (thin film) 3095, 2232, 1604, 1384, 1086, $791 \mathrm{~cm}^{-1}$; HRMS $\left(\mathrm{ESI}^{+}\right)[\mathrm{M}+\mathrm{H}]^{+}$calculated for $\mathrm{C}_{17} \mathrm{H}_{17} \mathrm{~N}_{2} \mathrm{OS}$ : 297.1062, found 297.1051. 


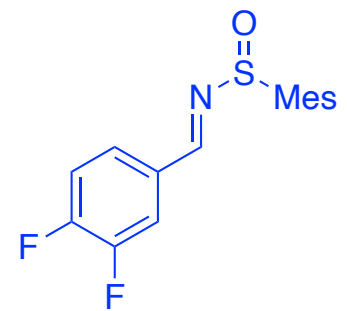

2,4,6-Trimethyl- $N$-(3,4-difluorobenzylidene)benzenesulfinamide (2f). Prepared via General Procedure B from 3,4-difluorobenzaldehyde (221 mg, $2.0 \mathrm{mmol}, 2.0$ equiv) and 2,4,6trimethylbenzenesulfinamide (366 mg, $2.0 \mathrm{mmol}, 2.0$ equiv) to give $\mathbf{2 f}$ (569 $\mathrm{mg}, 93 \%$ ) as a light yellow solid (mp: $\left.111-114{ }^{\circ} \mathrm{C}\right):{ }^{1} \mathrm{H} \mathrm{NMR}\left(600 \mathrm{MHz}, \mathrm{CDCl}_{3}\right) \delta 8.74(\mathrm{~s}, 1 \mathrm{H}), 7.73(\mathrm{ddd}, J=10.1$, 7.6, $2.1 \mathrm{~Hz}, 1 \mathrm{H}), 7.57(\mathrm{~m}, 1 \mathrm{H}), 7.28-7.23(\mathrm{~m}, 1 \mathrm{H}), 6.87(\mathrm{~s}, 2 \mathrm{H}), 2.49(\mathrm{~s}, 6 \mathrm{H}), 2.29(\mathrm{~s}, 3 \mathrm{H}) ;{ }^{13} \mathrm{C}$ NMR $\left(151 \mathrm{MHz}, \mathrm{CDCl}_{3}\right) \delta 159.4,153.3\left(\mathrm{dd}, J_{\mathrm{C}-\mathrm{F}}=268.8,12.1 \mathrm{~Hz}\right), 160.0 \mathrm{~d}\left(\mathrm{~d}, J_{\mathrm{C}-\mathrm{F}}=250.7,13.6\right.$ $\mathrm{Hz}), 142.1,138.6,135.1,131.3\left(\mathrm{app} \mathrm{t}, J_{\mathrm{C}-\mathrm{F}}=6.0 \mathrm{~Hz}\right), 131.1,126.9\left(\mathrm{dd}, J_{\mathrm{C}-\mathrm{F}}=7.6,4.5 \mathrm{~Hz}\right), 118.0$ $\left(\mathrm{d}, J_{\mathrm{C}-\mathrm{F}}=18.1 \mathrm{~Hz}\right), 117.7\left(\mathrm{~d}, J_{\mathrm{C}-\mathrm{F}}=18.1 \mathrm{~Hz}\right), 21.3,19.0 ;{ }^{19} \mathrm{~F}$ NMR $\left(565 \mathrm{MHz}, \mathrm{CDCl}_{3}\right) \delta-130.35$ (d, $J=22.6 \mathrm{~Hz}$ ), $-135.83\left(\mathrm{~d}, J=22.6 \mathrm{~Hz}\right.$ ), FTIR (thin film) 2922, 1583, 1515, 1434, 1282, 1087 $\mathrm{cm}^{-}$ 1; HRMS $\left(\mathrm{ESI}^{+}\right)[\mathrm{M}+\mathrm{H}]^{+}$calculated for $\mathrm{C}_{16} \mathrm{H}_{16} \mathrm{~F}_{2} \mathrm{NOS}$ : 308.0921, found 308.0908.

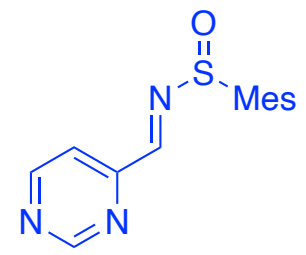

2,4,6-Trimethyl- $N$-(4-pyrimidinylmethylene)benzenesulfinamide (2g). Prepared via General Procedure B from 4-pyrimidinecarboxaldehyde $(0.18 \mathrm{~mL}, 2.0 \mathrm{mmol}, 2.0$ equiv) and 2,4,6trimethylbenzenesulfinamide (366 mg, $2.0 \mathrm{mmol}, 2.0$ equiv) to give $\mathbf{2 g}$ (529 $\mathrm{mg}, 97 \%$ ) as a white solid (mp: $\left.97-100{ }^{\circ} \mathrm{C}\right):{ }^{1} \mathrm{H}$ NMR $\left(600 \mathrm{MHz}, \mathrm{CDCl}_{3}\right) \delta 9.38(\mathrm{~d}, J=1.5 \mathrm{~Hz}, 1 \mathrm{H}), 8.89-8.89(\mathrm{~m}$, 2H), $7.91(\mathrm{~d}, J=4.9 \mathrm{~Hz}, 1 \mathrm{H}), 6.88(\mathrm{~s}, 2 \mathrm{H}), 2.50$ (s, 6H), 2.29 (s, 3H); ${ }^{13} \mathrm{C} \mathrm{NMR}\left(101 \mathrm{MHz}, \mathrm{CDCl}_{3}\right)$ $\delta 161.0,159.7,158.5,158.4,142.4,138.8,134.1,131.2,119.2,21.3,19.1$; FTIR (thin film) 2971, 1573, 1461, 1384, $1098 \mathrm{~cm}^{-1}$; HRMS $\left(\mathrm{ESI}^{+}\right)[\mathrm{M}+\mathrm{H}]^{+}$calculated for $\mathrm{C}_{14} \mathrm{H}_{16} \mathrm{~N}_{3} \mathrm{OS}: 274.1014$, found 274.1004. 


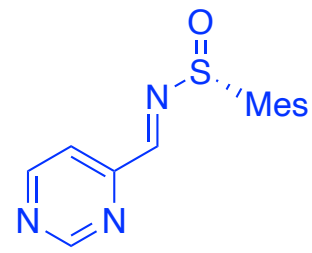

(R)-2,4,6-Trimethyl- $N$-(4-pyrimidinylmethylene)benzenesulfinamide $((\boldsymbol{R})-2 \mathrm{~g})$. Prepared via General Procedure B from 4-pyrimidinecarboxaldehyde (0.09 mL, $1.0 \mathrm{mmol}, 2.0$ equiv) and 2,4,6trimethylbenzenesulfinamide (183 $\mathrm{mg}, 1.0 \mathrm{mmol}, 2.0$ equiv) to give $(\boldsymbol{R})-\mathbf{2 g}(259 \mathrm{mg}, 95 \%)$ as a white solid that had analytical data consistent with that noted above. $[\alpha]_{D}^{22}=-133.4(\mathrm{c}=0.19$, $\left.\mathrm{CHCl}_{3}\right)$.

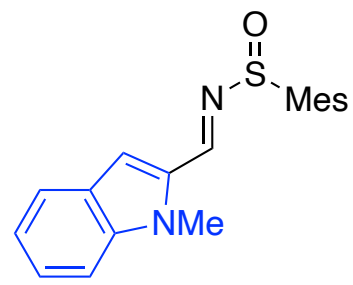

2,4,6-Trimethyl- $N$-[(1-methyl-1H-indol-2-yl)methylene]benzenesulfinamide (2j). Prepared via General Procedure B from 1H-indole-2-carbaldehyde (382 mg 2.4 mmol, 1.2 equiv) and 2,4,6trimethylbenzenesulfinamide (366 mg, $2.0 \mathrm{mmol}, 2.0$ equiv). The crude mixture was purified by silica gel chromatography $(50 \% \rightarrow 80 \%$ EtOAc/hexanes) to give $\mathbf{2} \mathbf{j}(648 \mathrm{mg}, 82 \%)$ as a light brown solid (mp: $\left.133-136{ }^{\circ} \mathrm{C}\right):{ }^{1} \mathrm{H}$ NMR $\left(600 \mathrm{MHz} \mathrm{CDCl}_{3}\right) \delta 8.87(\mathrm{~s}, 1 \mathrm{H}), 7.69(\mathrm{dt}, J=8.0,1.0$ $\mathrm{Hz}, 1 \mathrm{H}), 7.37-7.35(\mathrm{~m}, 2 \mathrm{H}), 7.15(\mathrm{dt}, J=8.0,3.9 \mathrm{~Hz}, 1 \mathrm{H}), 7.11(\mathrm{~s}, 1 \mathrm{H}), 6.89-6.86(\mathrm{~m}, 2 \mathrm{H}), 4.04$ (s, 3H), $2.52(\mathrm{~s}, 6 \mathrm{H}), 2.29(\mathrm{~s}, 3 \mathrm{H}) ;{ }^{13} \mathrm{C} \mathrm{NMR}\left(101 \mathrm{MHz}, \mathrm{CDCl}_{3}\right) \delta$ 153.1, 141.7, 141.0, 138.5, 136.0, 133.6, 131.0, 127.0, 125.7, 122.6, 120.8, 113.8, 110.1, 32.2, 21.3, 19.1; FTIR (thin film) 2962, 1594, 1524, 1469, 1352, 1084, $665 \mathrm{~cm}^{-1}$; HRMS $\left(\mathrm{ESI}^{+}\right)[\mathrm{M}+\mathrm{H}]^{+}$calculated for $\mathrm{C}_{19} \mathrm{H}_{21} \mathrm{~N}_{2} \mathrm{OS}$ : 325.1375 , found 325.1364 .

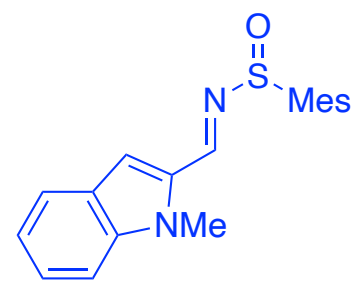

2,4,6-Trimethyl- $N$-(4-quinolinylmethylene)benzenesulfinamide (2k). Prepared via General Procedure B from 4-quinolinecarboxaldehyde (314 mg, $2.0 \mathrm{mmol}, 1.0$ equiv) and 2,4,6trimethylbenzenesulfinamide (366 mg, $2.0 \mathrm{mmol}, 1.0$ equiv) to give $\mathbf{2 k}(592 \mathrm{mg}, 92 \%)$ as a light yellow solid $\left(\operatorname{mp~} 99-101{ }^{\circ} \mathrm{C}\right):{ }^{1} \mathrm{H}$ NMR $\left(400 \mathrm{MHz}, \mathrm{CDCl}_{3}\right) \delta 9.51(\mathrm{~s}, 1 \mathrm{H}), 9.05(\mathrm{~d}, J=4.4 \mathrm{~Hz}$, 
$1 \mathrm{H}), 8.71(\mathrm{~d}, J=8.5 \mathrm{~Hz}, 1 \mathrm{H}), 8.22(\mathrm{~d}, J=8.5 \mathrm{~Hz}, 1 \mathrm{H}), 7.87(\mathrm{~d}, J=4.4 \mathrm{~Hz}, 1 \mathrm{H}), 7.81$ (ddd, $J=8.5$, 7.0, $1.5 \mathrm{~Hz}, 1 \mathrm{H}), 7.68(\mathrm{ddd}, J=8.5,6.9,1.5 \mathrm{~Hz}, 1 \mathrm{H}), 6.89(\mathrm{~s}, 2 \mathrm{H}), 2.54(\mathrm{~s}, 6 \mathrm{H}), 2.30(\mathrm{~s}, 3 \mathrm{H}) ;{ }^{13} \mathrm{C}$ NMR $\left(101 \mathrm{MHz}, \mathrm{CDCl}_{3}\right) \delta 159.2,150.3,149.1,142.3,138.7,136.5,134.6,131.2,131.0,130.4$, 130.1, 128.5, 125.5, 123.8, 121.9, 21.3, 19.1; FTIR: 3060, 1690, 1572, 1509, 1462, 1295, 1094, 849, $761 \mathrm{~cm}^{-1}$; HRMS $\left(\mathrm{ESI}^{+}\right)[\mathrm{M}+\mathrm{H}]^{+}$calculated for $\mathrm{C}_{19} \mathrm{H}_{19} \mathrm{~N}_{2} \mathrm{OS}: 323.1218$, found 323.1208.

\section{Preparation of Alkylpyridinium Salts}

If previously reported, the pyridinium salts were prepared as previously described: $\mathbf{1 a}$ and $\mathbf{1 i}{ }^{6}$ $\mathbf{1 b} ;{ }^{7} \mathbf{1 c}$ and $1 \mathrm{f} ;{ }^{8} \mathbf{1 d} ;{ }^{9} 1 \mathrm{e}$ and $\mathbf{1 h} ;{ }^{10} \mathbf{1 g},{ }^{11} \mathbf{1 j} ;{ }^{12} ; \mathbf{1 l} ;{ }^{13}$ and $\mathbf{1 m} .{ }^{14}$<smiles></smiles>

1 a<smiles></smiles>

$1 b$<smiles>c1ccc(-c2cc(-c3ccccc3)c(-c3ccccc3)c(-c3ccccc3)c2)cc1</smiles>

$1 c$

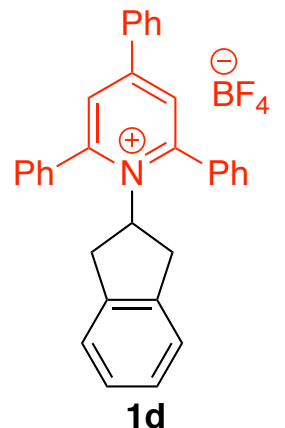

1d<smiles></smiles>

$1 e$<smiles></smiles>

$1 f$<smiles></smiles>

$1 \mathrm{~g}$<smiles></smiles>

1h<smiles></smiles>

$1 \mathrm{i}$<smiles></smiles>

1j<smiles></smiles><smiles></smiles><smiles></smiles> 


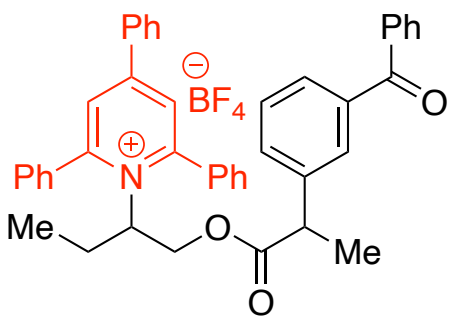

\section{1-(1-((2-(3-Benzoylphenyl)propanoyl)oxy)butan-2-yl)-2,4,6-triphenylpyridin-1-ium}

tetrafluoroborate (1k). To a round-bottomed flask, alkylpyridinium salt $1 \mathbf{m}(1 \mathrm{~g}, 2.1 \mathrm{mmol}, 1.0$ equiv), ketoprofen (636 mg, $2.5 \mathrm{mmol}, 1.2$ equiv), and dry DCM (0.1 M) was added. DCC (1.2 equiv) and DMAP (5 mol \%) were then added. The mixture stirred at room temperature overnight. The mixture was filtered through Celite ${ }^{\circledR}$ and concentrated. The resulting residue was purified by column chromatography $(2 \% \rightarrow 20 \% \mathrm{DCM} /$ Acetone $)$ to give $1 \mathbf{k}(1: 1$ diasteromers, $1.37 \mathrm{~g}, 91 \%)$ as a white solid $\left(\mathrm{mp} 87-91{ }^{\circ} \mathrm{C}\right):{ }^{1} \mathrm{H}$ NMR $\left(600 \mathrm{MHz}, \mathrm{CDCl}_{3}\right) \delta 7.92-7.30(\mathrm{~m}, 25 \mathrm{H}), 7.10(\mathrm{~d}, J$ $=13.9 \mathrm{~Hz}, 1 \mathrm{H}), 4.91-4.81(\mathrm{~m}, 1 \mathrm{H}), 4.03-3.96(\mathrm{~m}, 1 \mathrm{H}), 3.91(\mathrm{ddd}, J=12.1,7.6,4.3 \mathrm{~Hz}, 1 \mathrm{H})$, $3.75(\mathrm{dq}, J=39.5,7.1 \mathrm{~Hz}, 1 \mathrm{H}), 2.41-2.24(\mathrm{~m}, 1 \mathrm{H}), 1.56-1.46(\mathrm{~m}, 4 \mathrm{H}), 0.61(\mathrm{dt}, J=15.0,7.5$ $\mathrm{Hz}, 3 \mathrm{H}) ;{ }^{13} \mathrm{C} \mathrm{NMR}\left(151 \mathrm{MHz}, \mathrm{CDCl}_{3}\right) \delta 196.1,196.0,172.9,172.8,155.8,155.5,140.7,140.3$, $138.4,138.3,137.1,137.0,133.8,133.6,132.98,132.95,132.4,132.4,131.6,131.31,131.29$, 130.12 , 130.08, 129.8, 129.7, 129.5, 129.5, 128.8, 128.7, 128.6, 128.5, 128.5, 69.9, 69.8, 64.3, 64.2, 45.3, 45.1, 27.2, 26.9, 18.2, 18.1, 116, 11.5; ${ }^{19} \mathrm{~F}$ NMR (565 MHz, $\mathrm{CDCl}_{3}$ ) $\delta-153.14$ (minor, ${ }^{11} \mathrm{BF}_{4}$ ), -153.20 (major, ${ }^{10} \mathrm{BF}_{4}$ ); FTIR (thin film): 3429, 3058, 2977, 1739, 1654, 1619, 1283, 1072 , 766, 725, $702 \mathrm{~cm}^{-1}$; HRMS (ESI+) $\left[\mathrm{M}-\mathrm{BF}_{4}\right]^{+}$calculated for $\mathrm{C}_{43} \mathrm{H}_{38} \mathrm{NO}_{3}:$ 616.2846, found 616.2854 . 


\section{Mechanistic Experiments}

\section{UV/Vis Experiments}

The UV/Vis absorption spectra of DMA solutions of different combinations of alkylpyridinium 1a $(3 \mathrm{mM}), \mathrm{K}_{2} \mathrm{CO}_{3}(3 \mathrm{mM})$, sulfinimine $\mathbf{2 a}(1 \mathrm{mM})$, and $(\mathrm{TMS})_{3} \mathrm{SiOH}(1.5 \mathrm{mM})$ are shown in Figures S1-S3. The indicative red shift is seen in every experiment that includes both alkylpyridinium and $\mathrm{K}_{2} \mathrm{CO}_{3}$, indicating that the proposed anion $-\pi$ complex has formed. We also confirmed that triphenylpyridine (TPP, $0.003 \mathrm{M}$ ), a known byproduct of this reaction, is not causing the observed peak at $500 \mathrm{~nm}$.

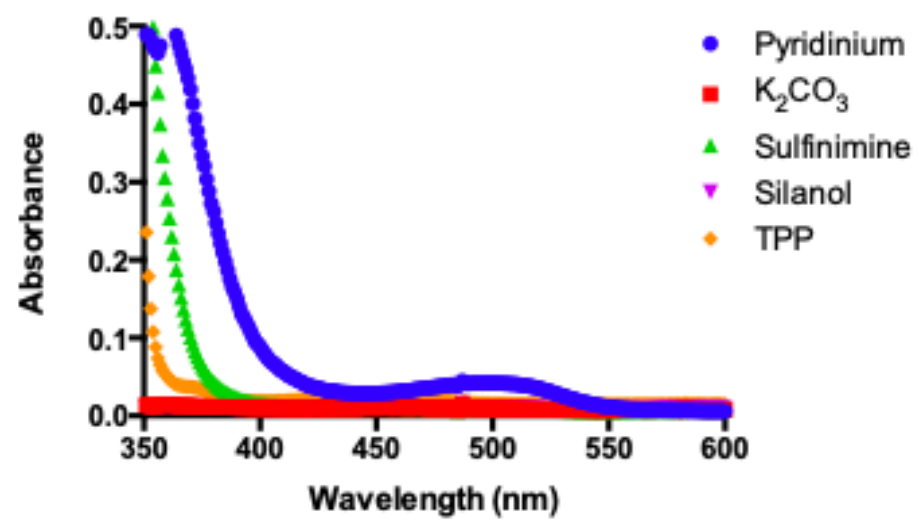

Figure S1. UV/Vis spectra of lone reaction components.

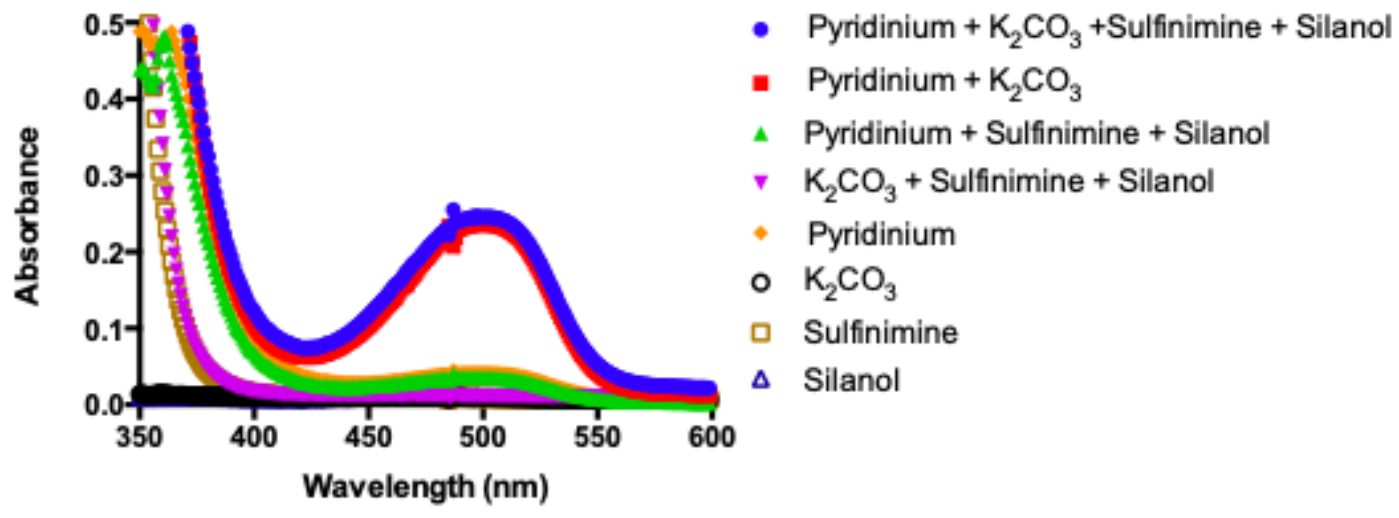

Figure S2. UV/Vis spectra of various combinations reaction components. 


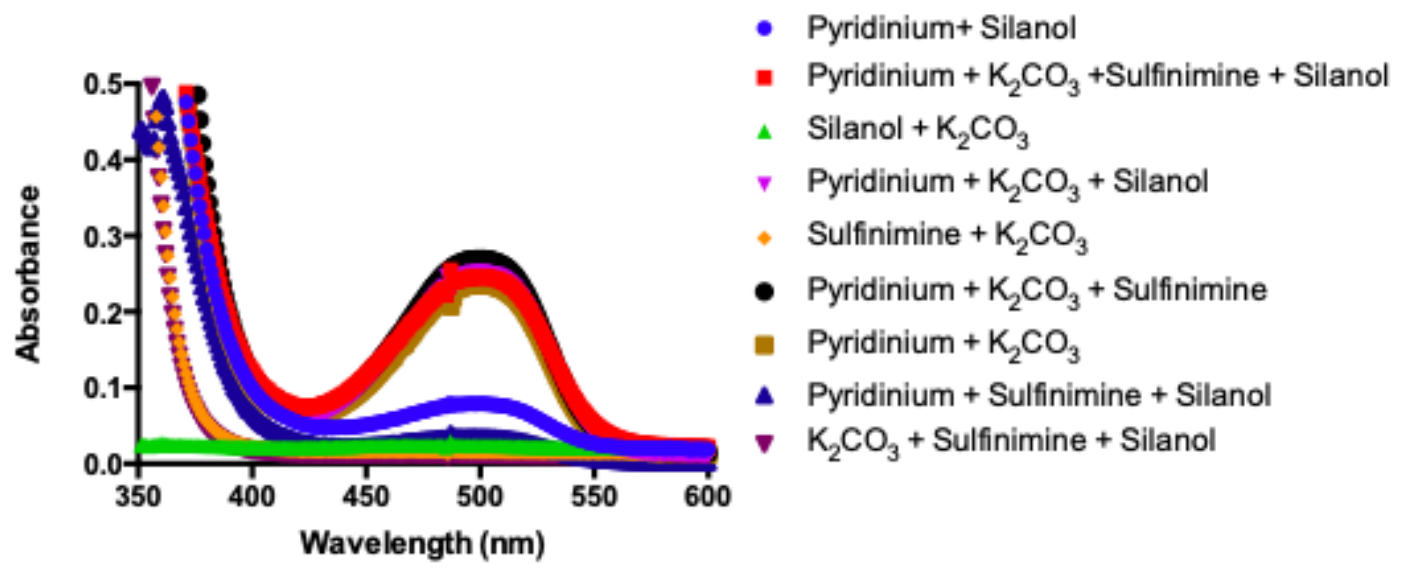

Figure S3. UV/Vis spectra of various combinations reaction components.

\section{Determination of the Stoichiometry of the Anion- $\pi$ Complex}

The stoichiometry of the anion $-\pi$ complex formed between pyridinium $\mathbf{1 a}$ and $\mathrm{K}_{2} \mathrm{CO}_{3}$ in $\mathrm{DMA}$ was determined by constructing Job's plot by measuring the absorption at $500 \mathrm{~nm}$ of DMA solutions of different ratios of $1 \mathbf{a}$ and $\mathrm{K}_{2} \mathrm{CO}_{3}$, where the total concentration of the two components remained constant at $1.0 \mathrm{mM}$.

\begin{tabular}{ccccc} 
entry & 1a: $\mathrm{K}_{2} \mathrm{CO}_{3}$ ratio & $\begin{array}{r}\text { Molar fraction } \\
\text { of 1a }(\%)\end{array}$ & $\begin{array}{c}\text { Absorbance at } \\
500 \mathrm{~nm}\end{array}$ & $\begin{array}{c}\text { Absorbance corrected for } \\
\text { molar fraction of 1a }\end{array}$ \\
\hline 1 & $1.0: 0.0$ & 100 & 0.04213 & 0 \\
2 & $0.8: 0.2$ & 80 & 0.10013 & 0.020026 \\
3 & $07: 0.3$ & 70 & 0.117 & 0.0351 \\
4 & $0.6: 0.4$ & 60 & 0.18673 & 0.074692 \\
5 & $0.5: 0.5$ & 50 & 0.23629 & 0.118145 \\
6 & $0.4: 0.6$ & 40 & 0.15459 & 0.092754 \\
7 & $0.3: 0.7$ & 30 & 0.099108 & 0.0693756 \\
8 & $0.2: 0.8$ & 20 & 0.07387 & 0.059096 \\
9 & $0.0: 1.0$ & 0 & 0.00957 & 0.00957
\end{tabular}

The absorbance values were corrected with respect to molar fraction of 1a and plotted against the molar fraction 1 a. 


\section{Job Plot}

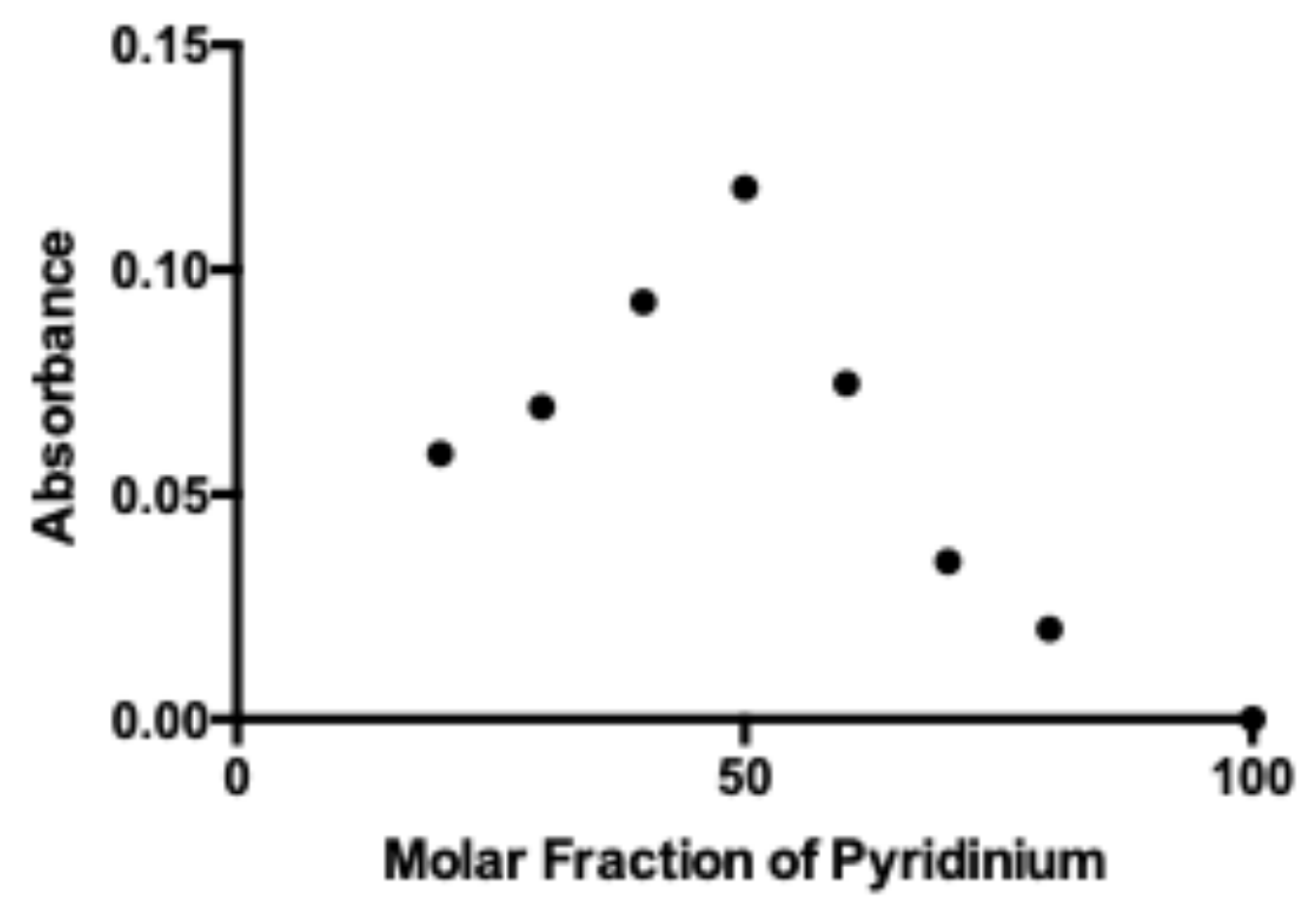

The maximum absorbance is obtained at 49\% molar fraction. This indicates the stoichiometry of the anion $-\pi$ complex is $1: 1$.

\section{Radical Trap Experiments}

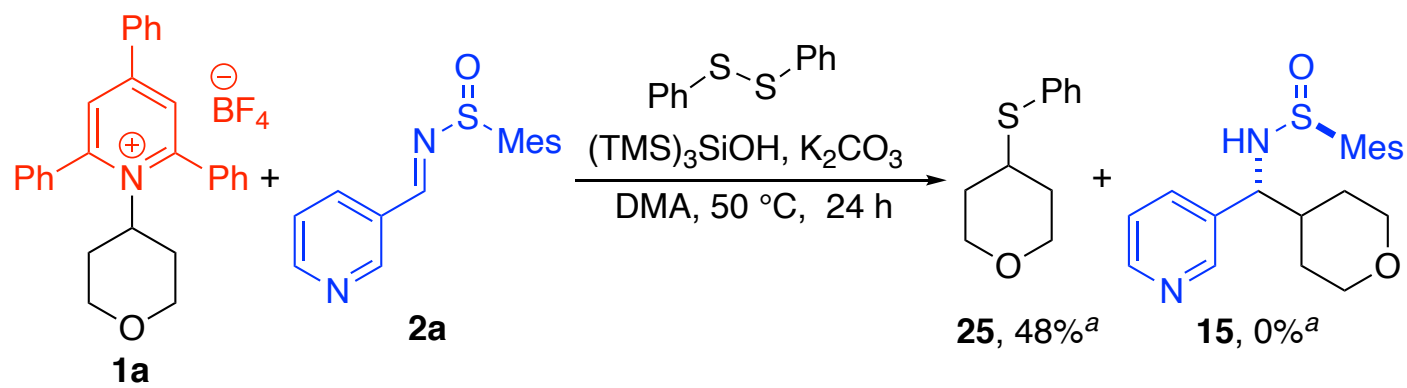

In a $\mathrm{N}_{2}$-atmosphere glovebox: alkylpyridinium salt $1 \mathbf{a}(120.0 \mathrm{mg}, 0.25 \mathrm{mmol}, 2.5$ equiv), $2 \mathrm{a}$ (27.2 $\mathrm{mg}, 0.10 \mathrm{mmol}, 1.0$ equiv), $\mathrm{K}_{2} \mathrm{CO}_{3}$ (42 mg, $0.30 \mathrm{mmol}, 3.0$ equiv) and diphenyl disulfide (109 $\mathrm{mg}$, $0.50 \mathrm{mmol}, 5.0$ equiv) were added to an oven-dried 1-dram vial fitted with a stir bar. DMA (0.9 $\mathrm{mL}, 0.11 \mathrm{M}$ ) was added via syringe followed by $\mathrm{TMS}_{3} \mathrm{SiOH}(41 \mu \mathrm{L}, 0.15 \mathrm{mmol}, 1.5$ equiv). The vial was capped with a Teflon-lined cap and removed from the glovebox. The mixture was stirred for $24 \mathrm{~h}$ at $50{ }^{\circ} \mathrm{C}$ in an aluminum heating block. The mixture was then diluted with EtOAc $(3 \mathrm{~mL})$ 
and filtered through a plug of Celite ${ }^{\circledR}$, which was rinsed with EtOAc $(10 \mathrm{~mL})$. The filtrate was concentrated. 1,3,5-Trimethoxybenzene (internal standard) was added. $\mathrm{CDCl}_{3}$ was added, and the yield of 25 (and 3) was determined by ${ }^{1} \mathrm{H}$ NMR analysis. Please note: Sulfide 25 is commercially available, allowing comparison of the NMR of the crude reaction mixture to an authentic sample.

\section{Radical Trap Control Experiments}

A similar procedure was employed for the control experiments shown below.

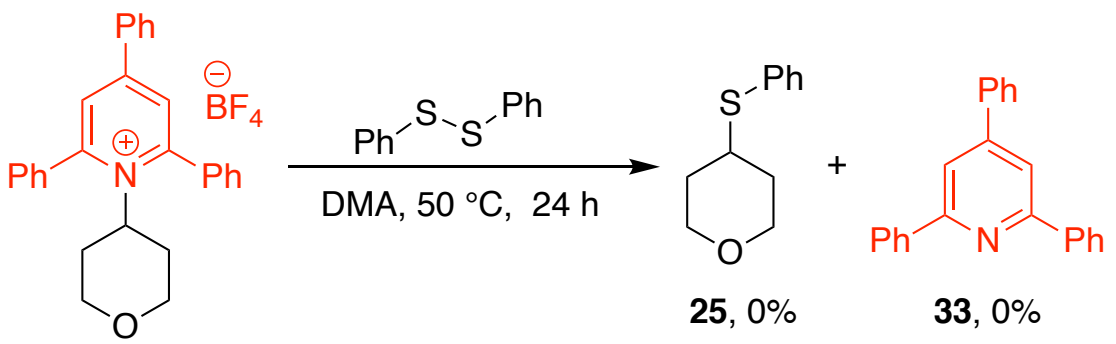

$1 a$

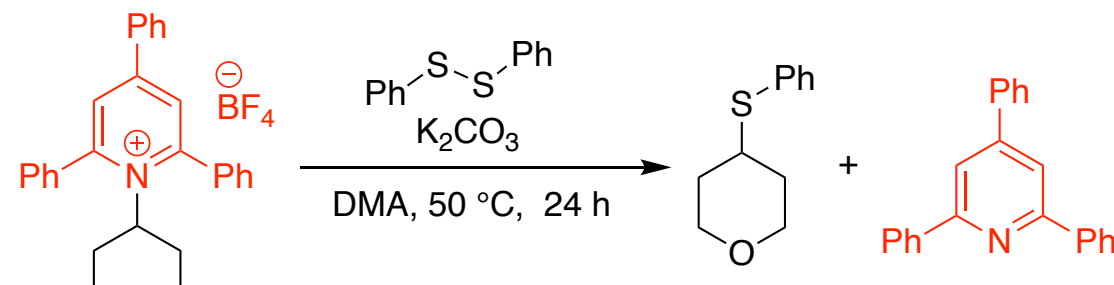

$25,26 \% \quad 33,27 \%$

$1 \mathrm{a}$

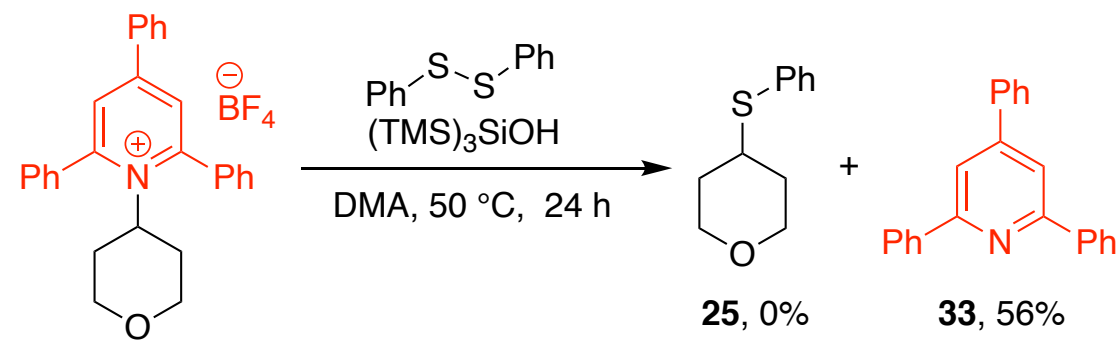

$1 \mathrm{a}$ 


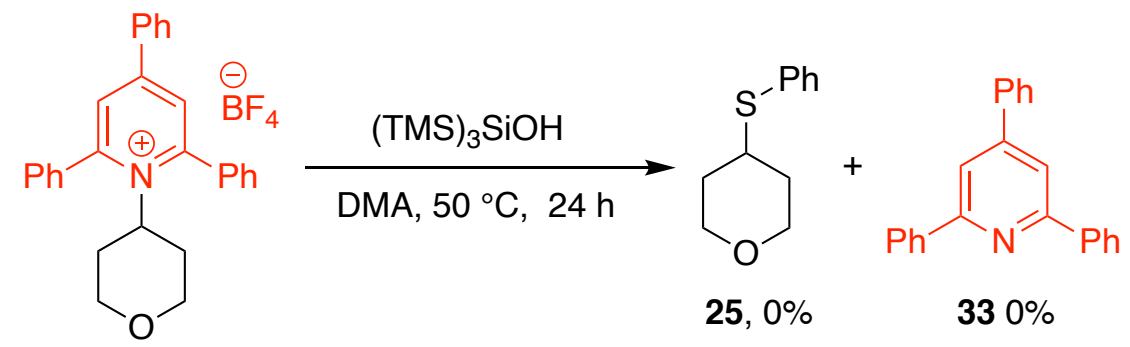

1 a

\section{Control Experiments Probing for Pyridinium Ylide Formation}

To probe for the possibility of deprotonation of the alkylpyridinium salt by $\mathrm{K}_{2} \mathrm{CO}_{3}$ (i.e., formation of an alkylpyridinium ylide), a deuterium-labelling study was performed. During this study, $\mathrm{D}_{2} \mathrm{O}$ was added to a mixture of pyridinium salt, $\mathrm{K}_{2} \mathrm{CO}_{3}$, and DMA either at the beginning or at the end. Both experiments resulted in $\geq 94 \%$ retention of the proton $\left(\mathrm{D}_{2} \mathrm{O}\right.$ addition at the beginning: $94 \%$ retention, $\mathrm{D}_{2} \mathrm{O}$ quench: $97 \%$ retention), suggesting that the reaction does not proceed through a ylide intermediate.

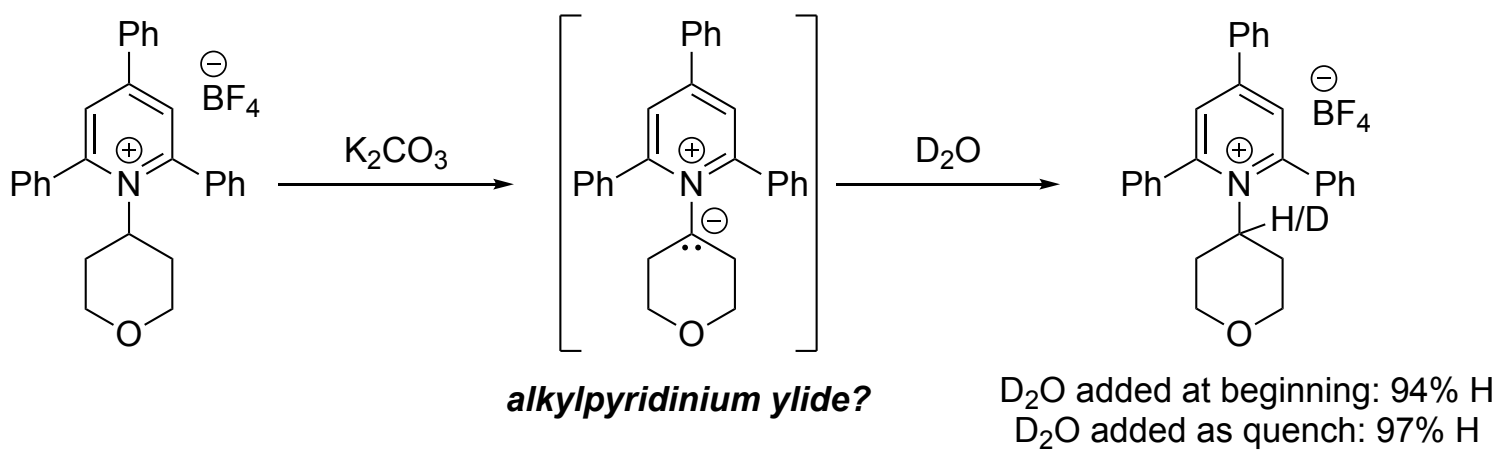

$\mathbf{D}_{2} \mathbf{O}$ Addition at Beginning. In a $\mathrm{N}_{2}$-atmosphere glovebox, alkylpyridinium salt 1a (69.0 mg, 0.2 mmol, 1.0 equiv), and $\mathrm{K}_{2} \mathrm{CO}_{3}$ (33.0 mg, $0.24 \mathrm{mmol}, 1.2$ equiv) were added to an oven-dried 1dram vial fitted with a stir bar. DMA $(1.8 \mathrm{~mL}, 0.11 \mathrm{M})$ was added via syringe. The vial was capped with a pierceable Teflon-lined cap and removed from the glovebox. $\mathrm{D}_{2} \mathrm{O}(10 \mu \mathrm{L}, 0.55 \mathrm{mmol}, 2.8$ equiv) was added, and the mixture was stirred for $24 \mathrm{~h}$ at $50{ }^{\circ} \mathrm{C}$ in an aluminum heating block. After $24 \mathrm{~h}$, the mixture was concentrated. $\mathrm{CDCl}_{3}$ was added, and the mixture was filtered through a pipette fitted with a Kimwipe ${ }^{\circledR}$ and sand into an NMR tube. By ${ }^{1} \mathrm{H}$ NMR spectroscopy, 94\% retention of the proton was observed.

$\mathbf{D}_{2} \mathbf{O}$ Quench. In a $\mathrm{N}_{2}$-atmosphere glovebox, alkylpyridinium salt 1a $(69.0 \mathrm{mg}, 0.2 \mathrm{mmol}, 1.0$ equiv), and $\mathrm{K}_{2} \mathrm{CO}_{3}(33.0 \mathrm{mg}, 0.24 \mathrm{mmol}, 1.2$ equiv) were added to an oven-dried 1-dram vial fitted 
with a stir bar. DMA $(1.8 \mathrm{~mL}, 0.11 \mathrm{M})$ was added via syringe. The vial was capped with a pierceable Teflon-lined cap and removed from the glovebox. The mixture was stirred for $24 \mathrm{~h}$ at $50{ }^{\circ} \mathrm{C}$. After $24 \mathrm{~h}$, the reaction was quenched by adding $\mathrm{D}_{2} \mathrm{O}(10 \mu \mathrm{L}, 0.55 \mathrm{mmol}, 2.8$ equiv $)$, and then the mixture was concentrated. $\mathrm{CDCl}_{3}$ was added, and the mixture was filtered through a pipette fitted with a Kimwipe ${ }^{\circledR}$ and sand into an NMR tube. By ${ }^{1} \mathrm{H}$ NMR spectroscopy, 97\% retention of the proton was observed.

\section{References}

1. Pangborn, A. B.; Giardello, M. A.; Grubbs, R. H.; Rosen, R. K.; Timmers, F. J., Safe and convenient procedure for solvent purification. Organometallics 1996, 15 (5), 1518-1520.

2. Garrido-Castro, A. F.; Choubane, H.; Daaou, M.; Maestro, M. C.; Alemán, J., Asymmetric radical alkylation of N-sulfinimines under visible light photocatalytic conditions. Chem. Commun. 2017, 53 (55), $7764-7767$.

3. Backes, B. J.; Dragoli, D. R.; Ellman, J. A., Chiral N-Acyl-tert-butanesulfinamides: The "Safety-Catch" Principle Applied to Diastereoselective Enolate Alkylations. J. Org. Chem. 1999, 64 (15), 5472-5478.

4. Morales, S.; Guijarro, F. G.; García Ruano, J. L.; Cid, M. B., A General Aminocatalytic Method for the Synthesis of Aldimines. J. Am. Chem. Soc. 2014, 136 (3), 1082-1089.

5. Ni, S.; Padial, N. M.; Kingston, C.; Vantourout, J. C.; Schmitt, D. C.; Edwards, J. T.; Kruszyk, M. M.; Merchant, R. R.; Mykhailiuk, P. K.; Sanchez, B. B.; Yang, S.; Perry, M. A.; Gallego, G. M.; Mousseau, J. J.; Collins, M. R.; Cherney, R. J.; Lebed, P. S.; Chen, J. S.; Qin, T.; Baran, P. S., A Radical Approach to Anionic Chemistry: Synthesis of Ketones, Alcohols, and Amines. J. Am. Chem. Soc. 2019, 141 (16), 6726-6739.

6. Plunkett, S.; Basch, C. H.; Santana, S. O.; Watson, M. P., Harnessing Alkyl Pyridinium Salts as Electrophiles in De-aminative Alkyl-Alkyl Cross-Couplings. J. Am. Chem. Soc. 2019, 141 (6), $2257-2262$.

7. Yue, H.; Zhu, C.; Shen, L.; Geng, Q.; Hock, K. J.; Yuan, T.; Cavallo, L.; Rueping, M., Nickel-catalyzed $\mathrm{C}-\mathrm{N}$ bond activation: activated primary amines as alkylating reagents in reductive cross-coupling. Chem. Sci. 2019, $10,4430-4435$.

8. Katritzky, A. R.; De Ville, G.; Patel, R. C., Carbon-alkylation of simple nitronate anions by N-substituted pyridiniums. Tetrahedron 1981, 37, 25-30.

9. Wu, J.; He, L.; Noble, A.; Aggarwal, V. K., Photoinduced Deaminative Borylation of Alkylamines. J. Am. Chem. Soc. 2018, 140 (34), 10700-10704.

10. Basch, C. H.; Liao, J.; Xu, J.; Piane, J. J.; Watson, M. P., Harnessing Alkyl Amines as Electrophiles for Nickel-Catalyzed Cross Couplings via C-N Bond Activation. J. Am. Chem. Soc. 2017, 139 (15), 5313-5316.

11. Liao, J.; Basch, C. H.; Hoerrner, M. E.; Talley, M. R.; Boscoe, B. P.; Tucker, J. W.; Garnsey, M. R.; Watson, M. P., Deaminative Reductive Cross-Electrophile Couplings of Alkylpyridinium Salts and Aryl Bromides. Org Lett 2019, 21 (8), 2941-2946.

12. Yi, J.; Badir, S. O.; Kammer, L. M.; Ribagorda, M.; Molander, G. A., Deaminative Reductive Arylation Enabled by Nickel/Photoredox Dual Catalysis. Org. Lett. 2019, 21 (9), 3346-3351.

13. Martin-Montero, R.; Yatham, V. R.; Yin, H.; Davies, J.; Martin, R., Ni-catalyzed Reductive Deaminative Arylation at sp(3) Carbon Centers. Org Lett 2019, 21 (8), 2947-2951.

14. Sun, S.-Z.; Romano, C.; Martin, R., Site-Selective Catalytic Deaminative Alkylation of Unactivated Olefins. J. Am. Chem. Soc. 2019, 141 (41), 16197-16201. 


\section{SFC Traces}

\section{Compound 10, racemic}

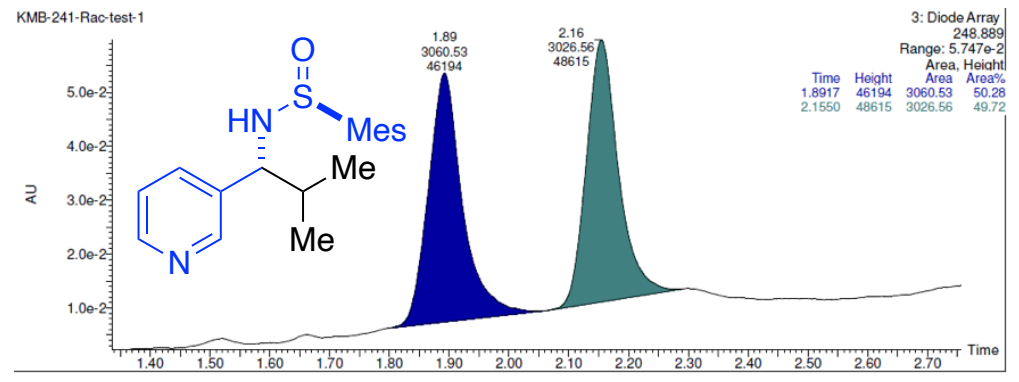

Compound $(\boldsymbol{R}, \boldsymbol{R})-\mathbf{1 0},>99 \%$ ee

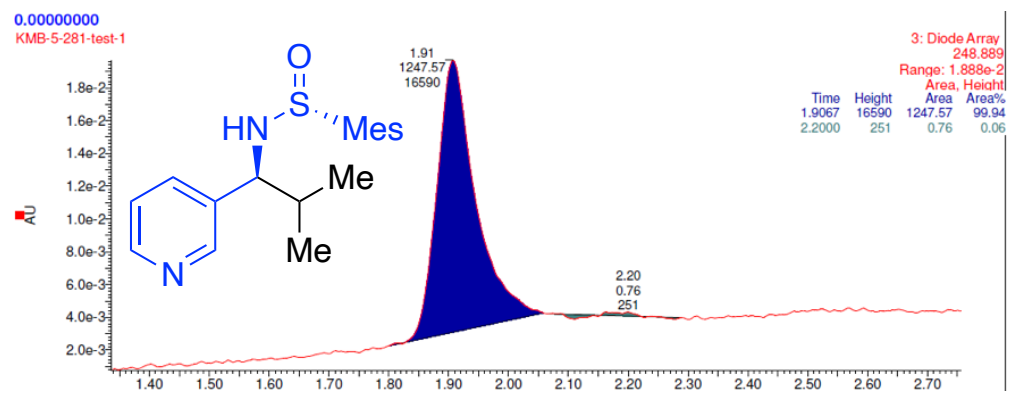


Compound 15, racemic

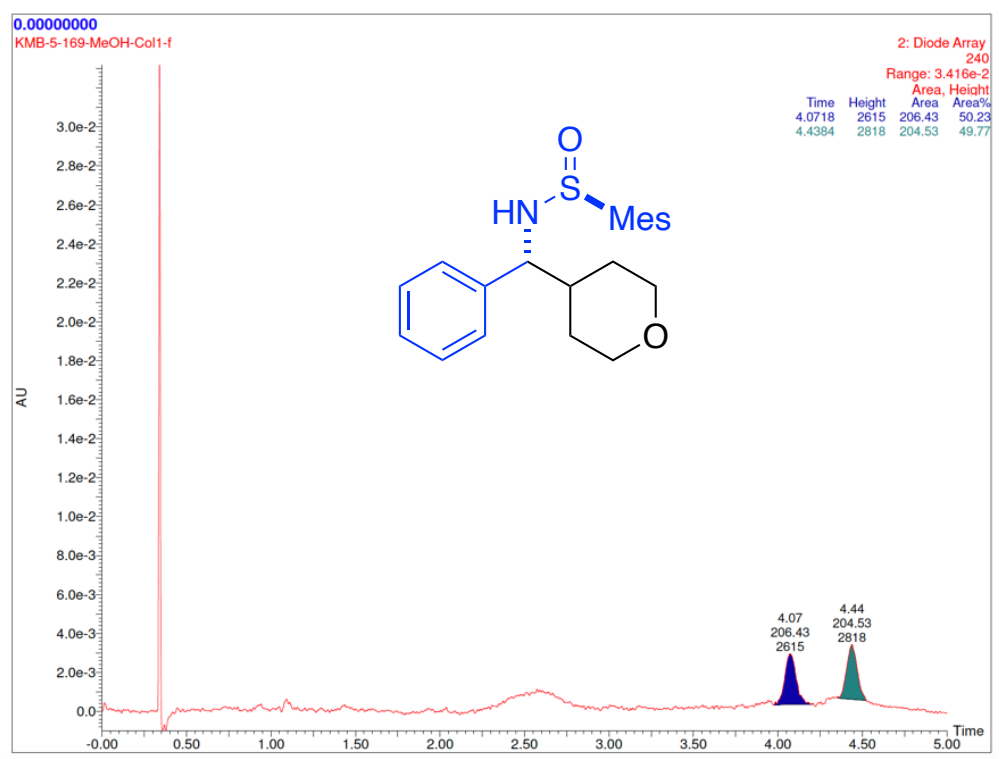

Compound $(S, S)-15,>99 \%$ ee

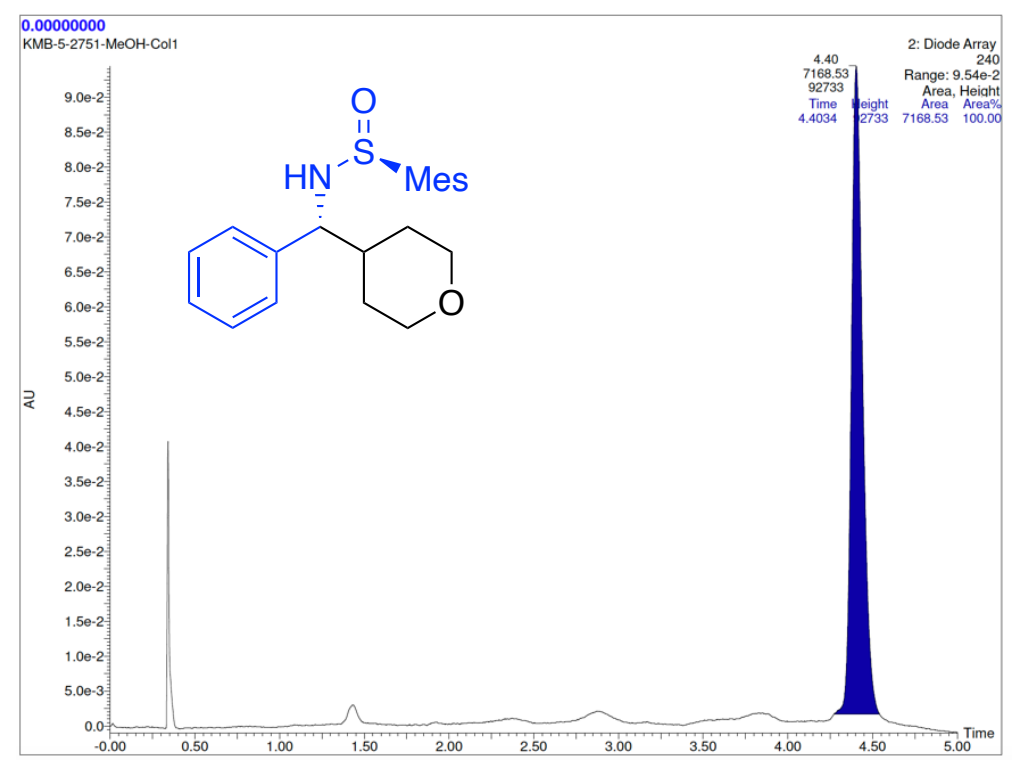


Compound 17, racemic

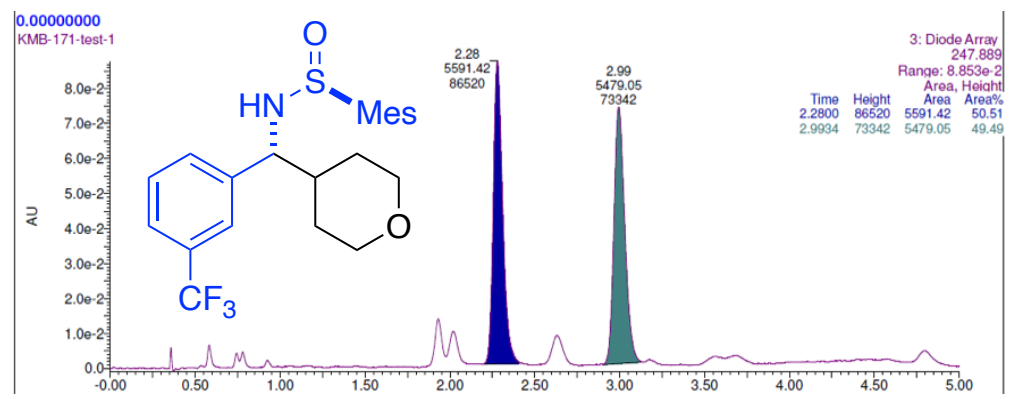

Compound $(S, S)-17,>99 \%$ ee

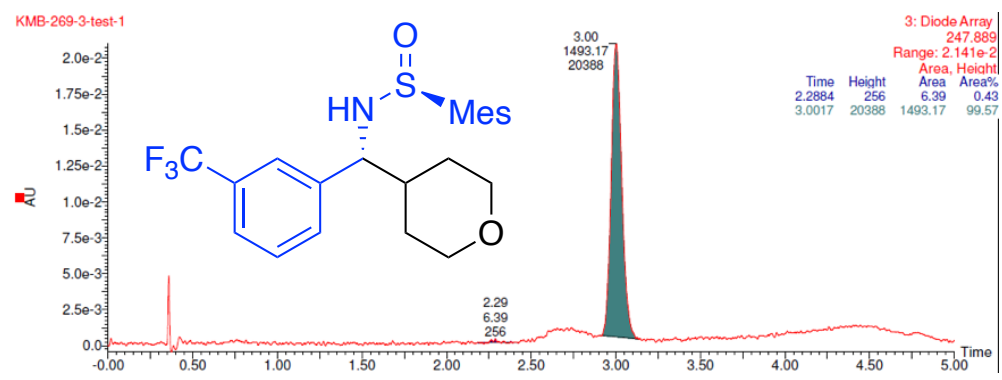


Compound 20, racemic

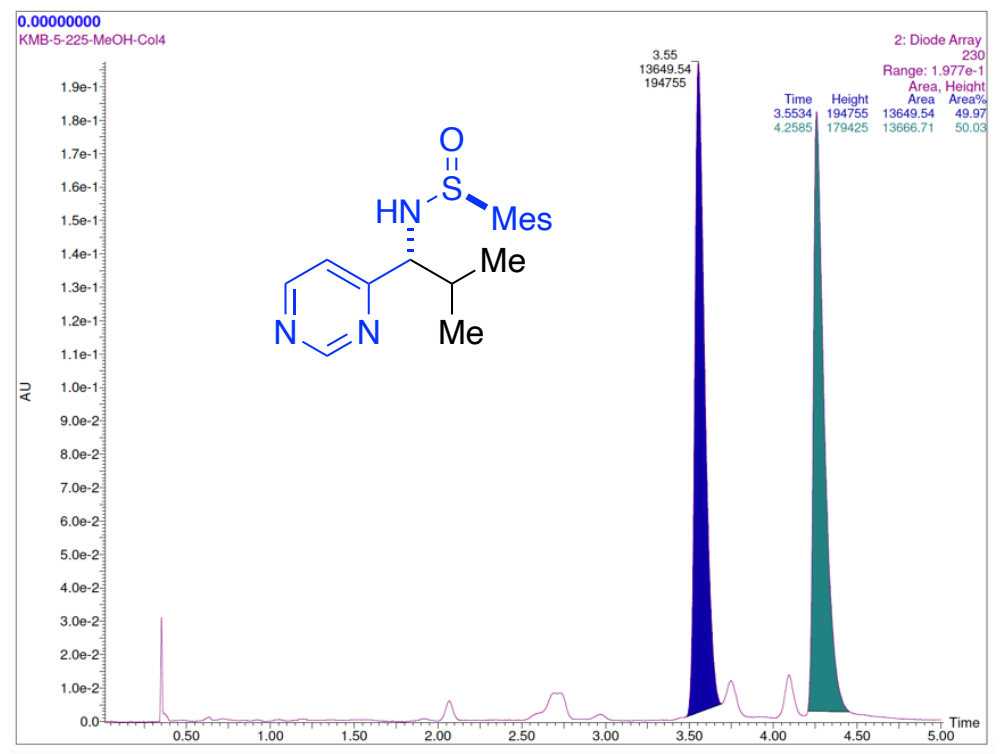

Compound $(\boldsymbol{R}, \boldsymbol{R})-\mathbf{2 0},>99 \%$ ee

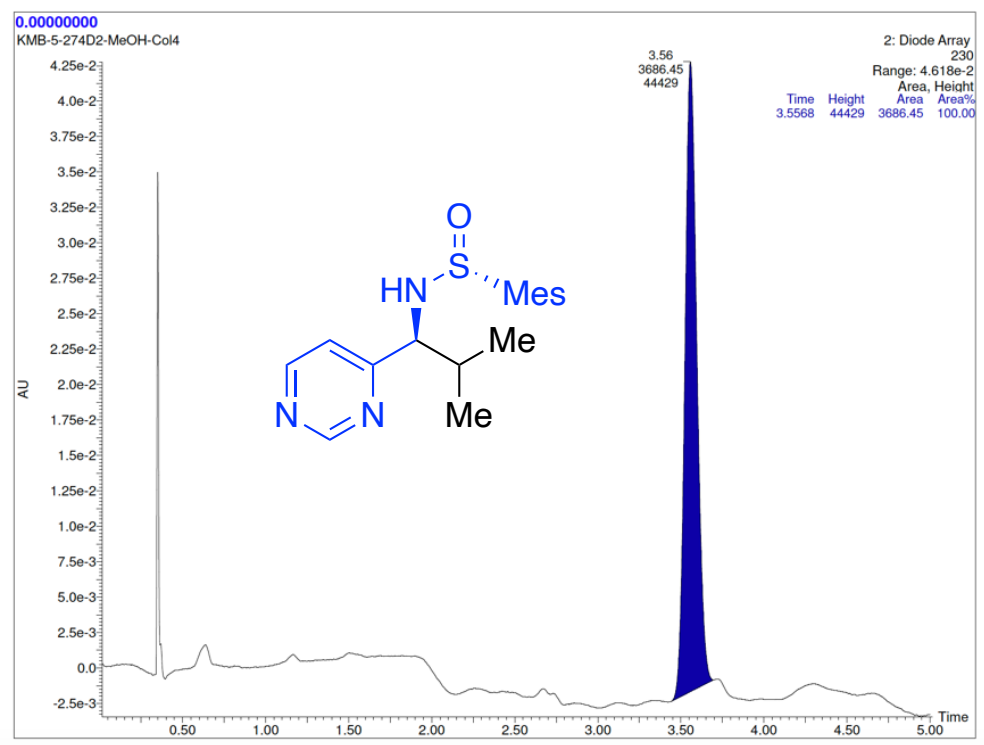




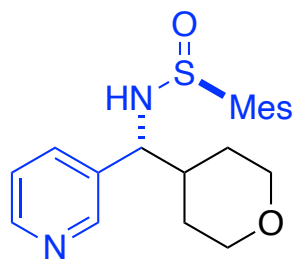

Compound 3

${ }^{1} \mathrm{H}$ NMR $\left(400 \mathrm{MHz}, \mathrm{CDCl}_{3}\right)$

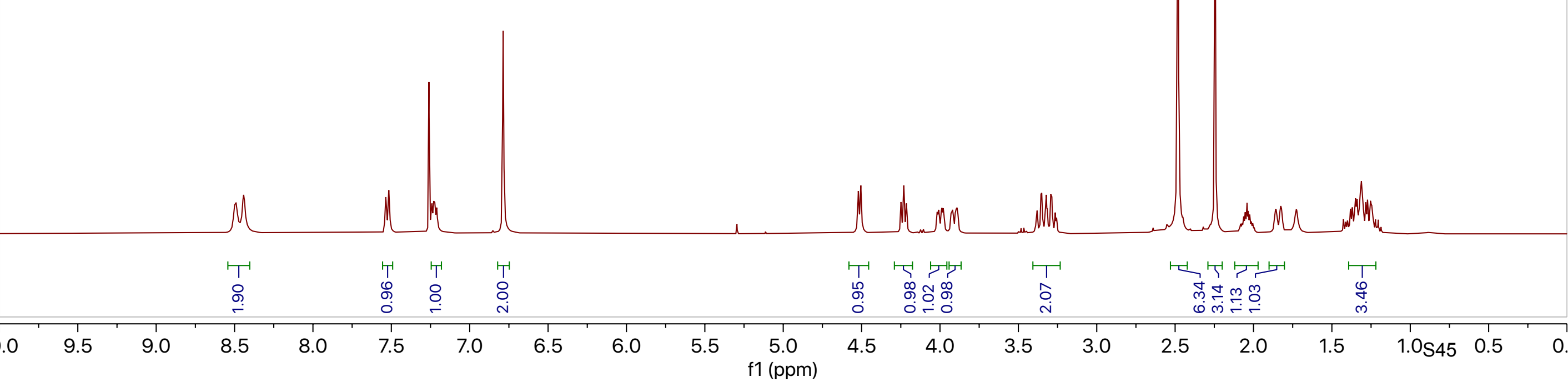



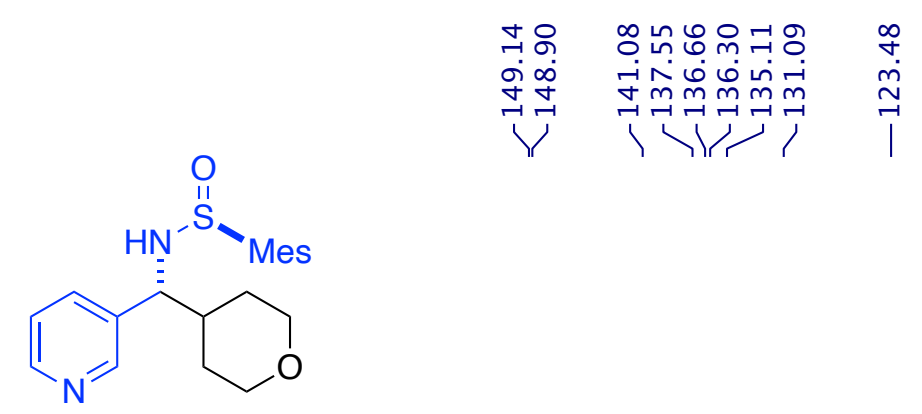

Compound 3

${ }^{13} \mathrm{C}$ NMR (151 MHz, $\mathrm{CDCl}_{3}$ )

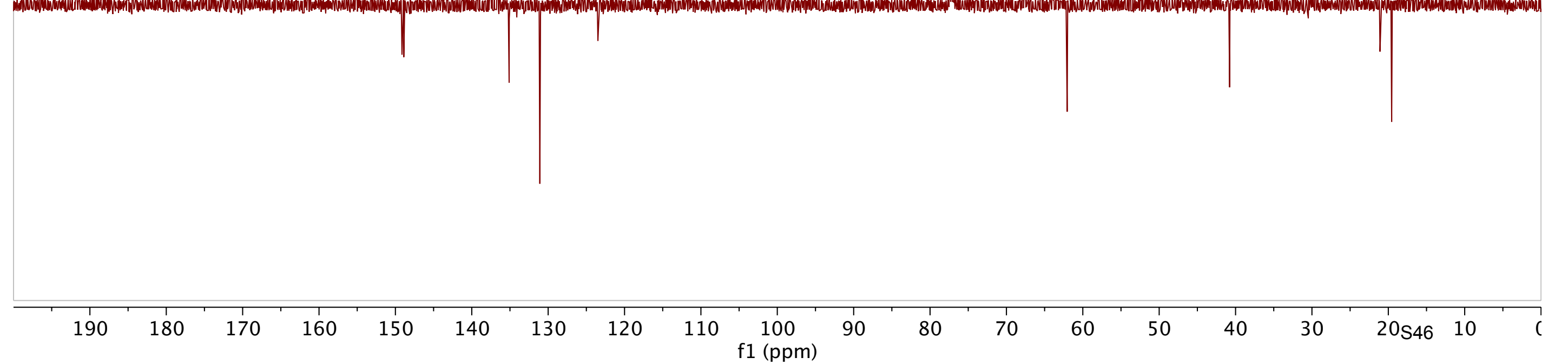




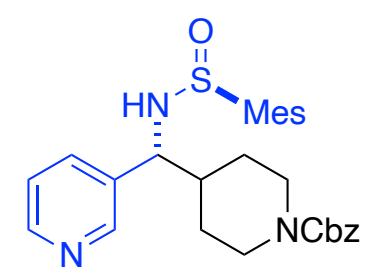

Compound 4

${ }^{1} \mathrm{H} \mathrm{NMR}\left(600 \mathrm{MHz}, \mathrm{CDCl}_{3}\right)$

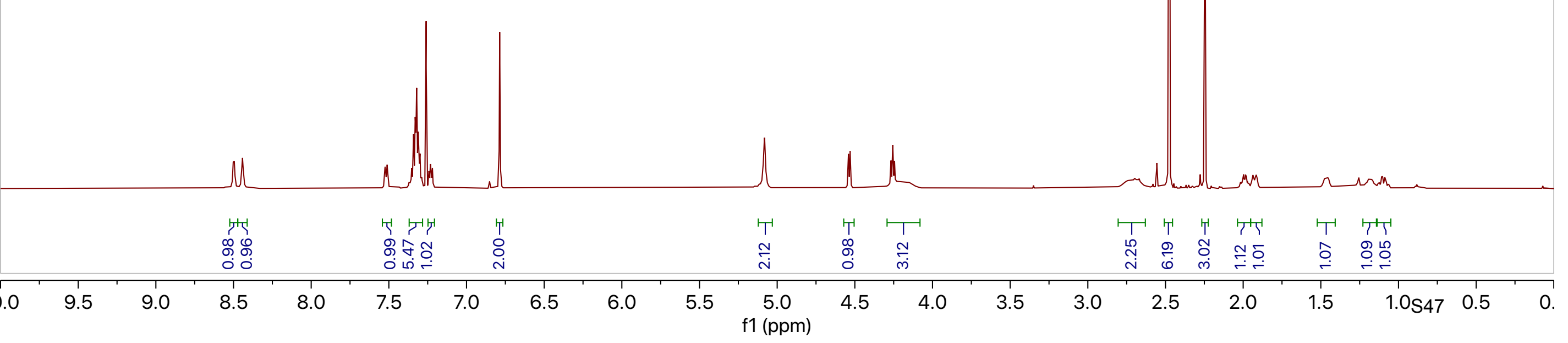




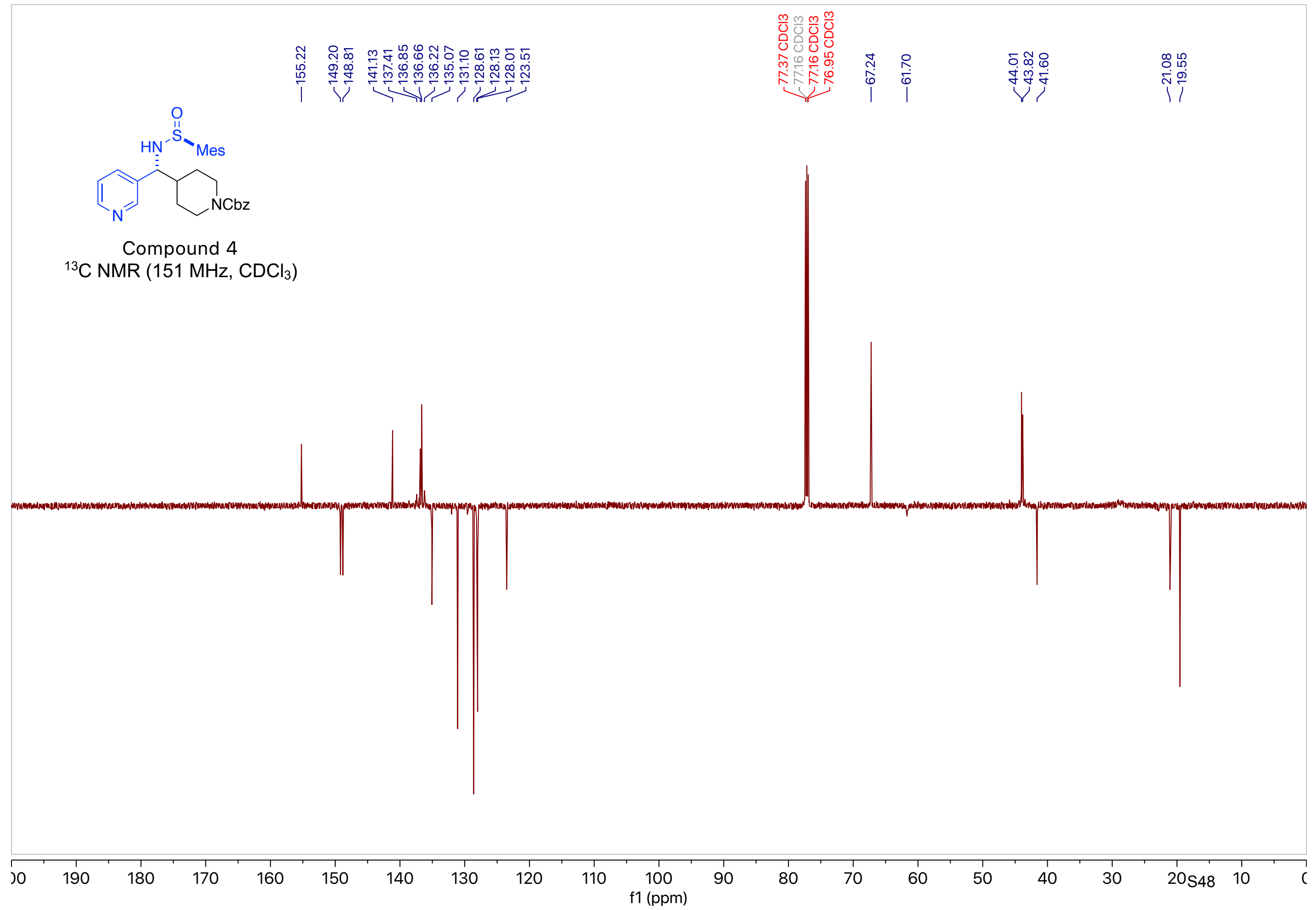




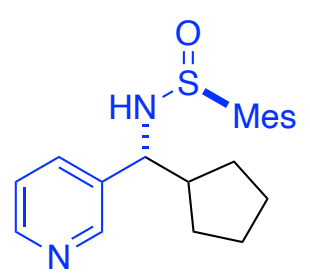

Compound 5

${ }^{1} \mathrm{H}$ NMR $\left(600 \mathrm{MHz}, \mathrm{CDCl}_{3}\right)$ 


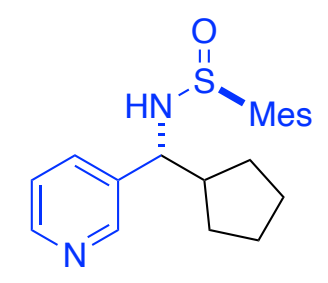

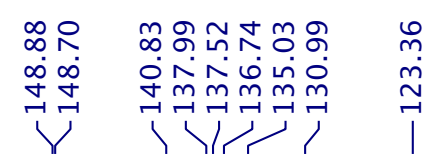

Compound 5

${ }^{13} \mathrm{C}$ NMR (151 MHz, $\mathrm{CDCl}_{3}$ )

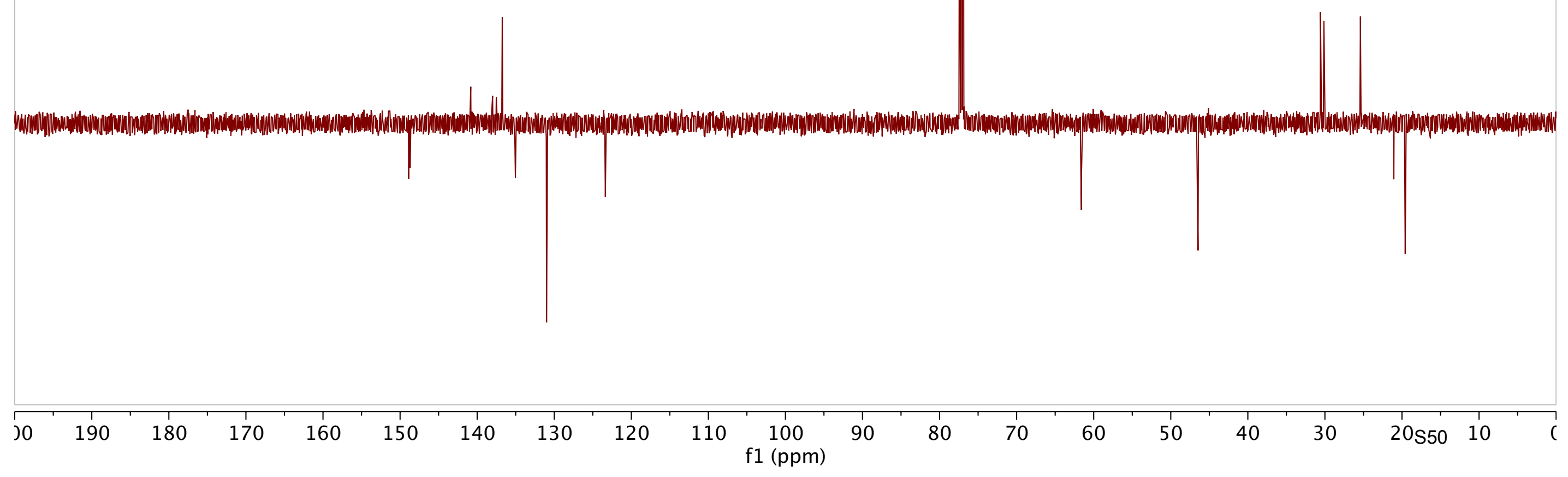




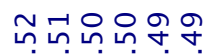

$\infty \infty \infty \infty \infty \infty$

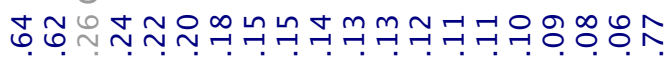

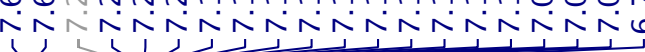

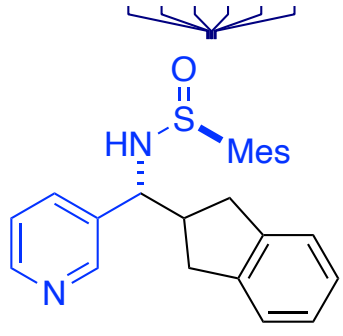

Compound 6

${ }^{1} \mathrm{H}$ NMR $\left(400 \mathrm{MHz}, \mathrm{CDCl}_{3}\right)$

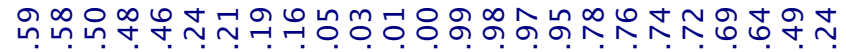

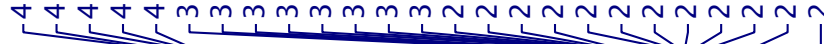

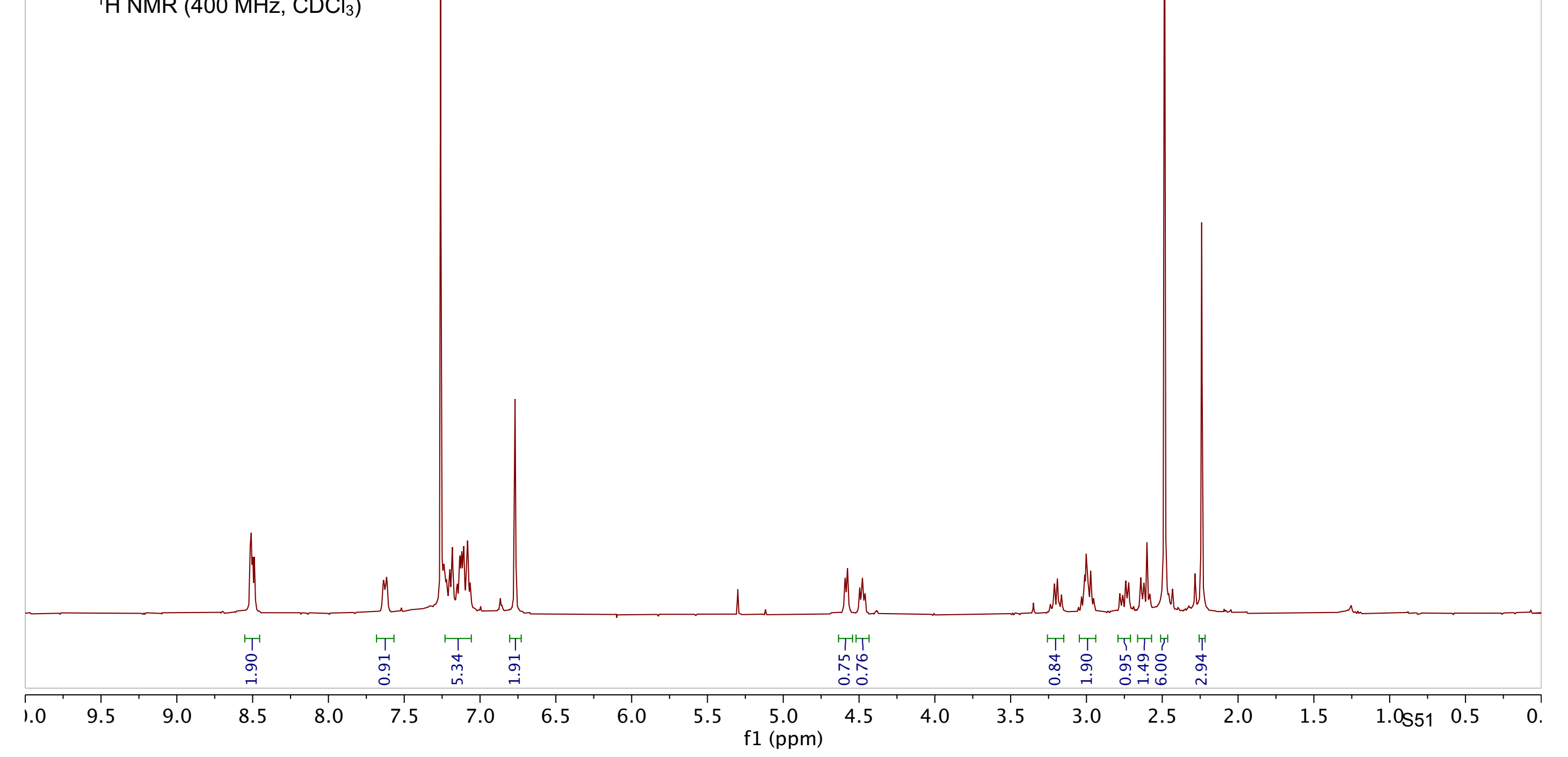




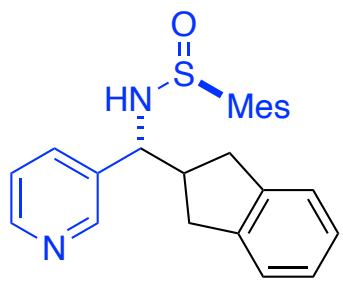

Compound 6

${ }^{13} \mathrm{C}$ NMR (101 MHz, $\mathrm{CDCl}_{3}$ )

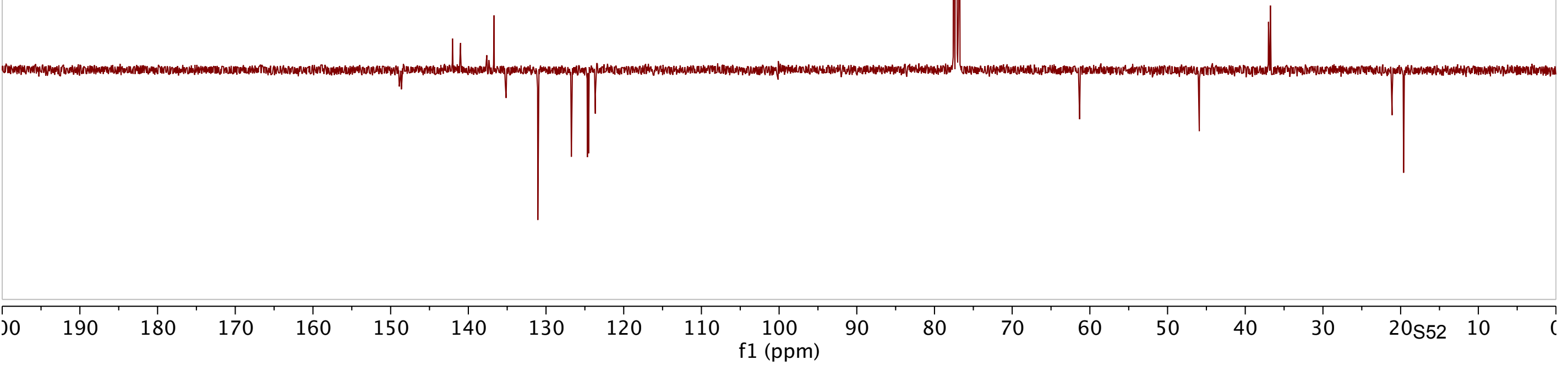




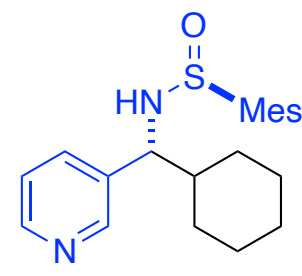

Compound 7

${ }^{1} \mathrm{H} \mathrm{NMR}\left(600 \mathrm{MHz}, \mathrm{CDCl}_{3}\right)$

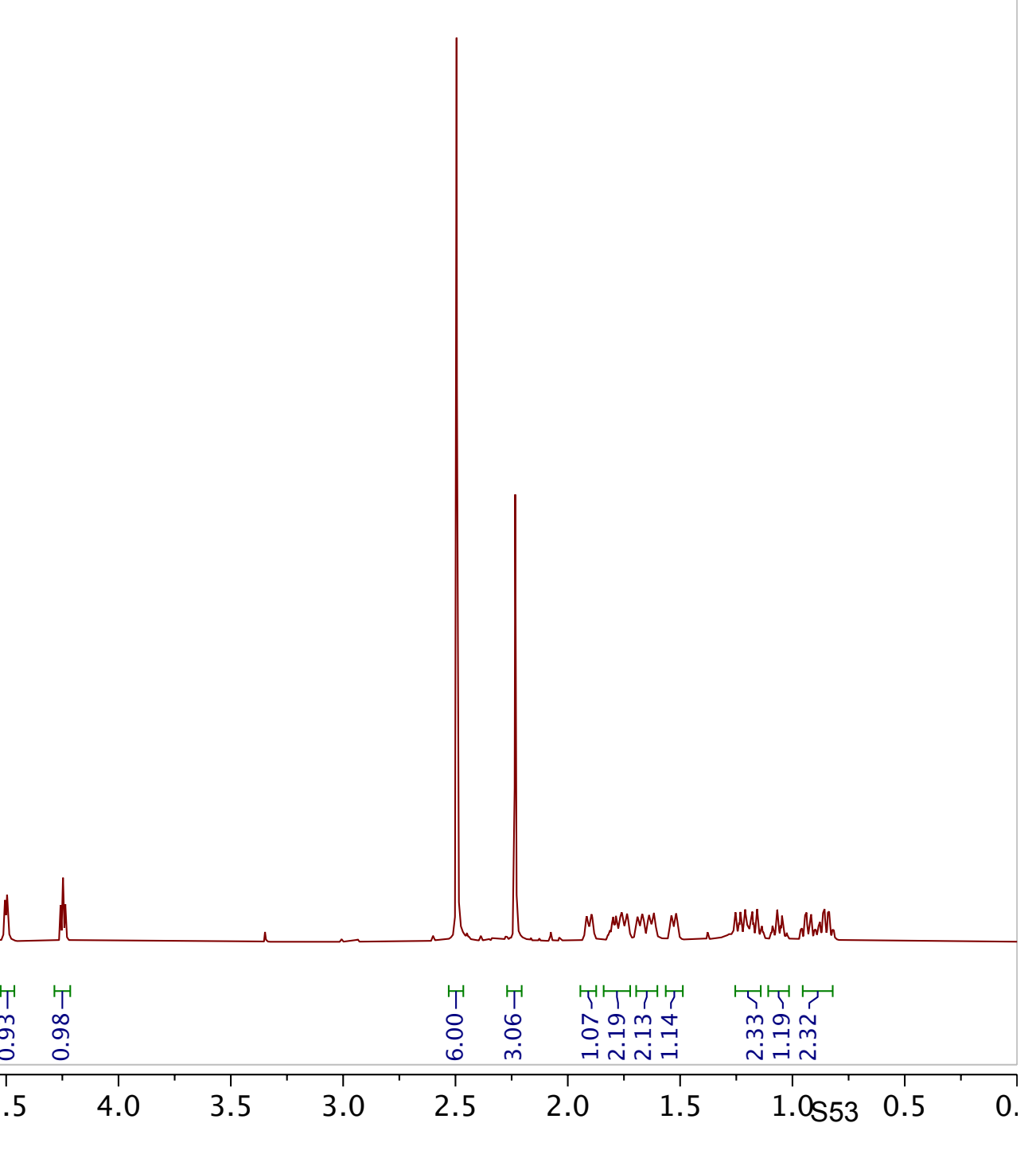



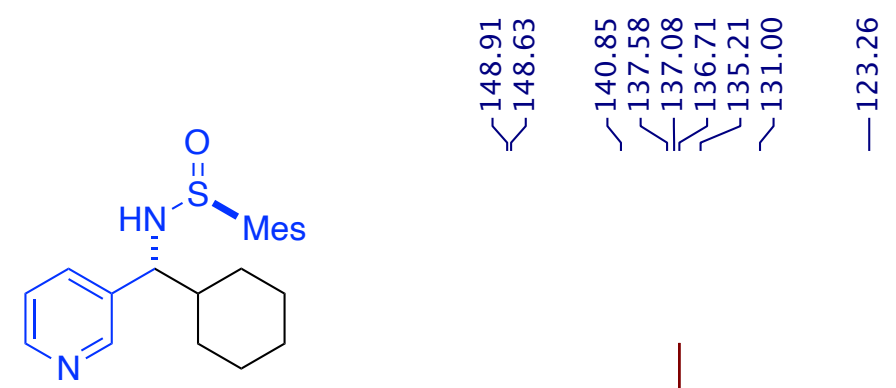

Compound 7

${ }^{13} \mathrm{C}$ NMR (151 MHz, $\left.\mathrm{CDCl}_{3}\right)$

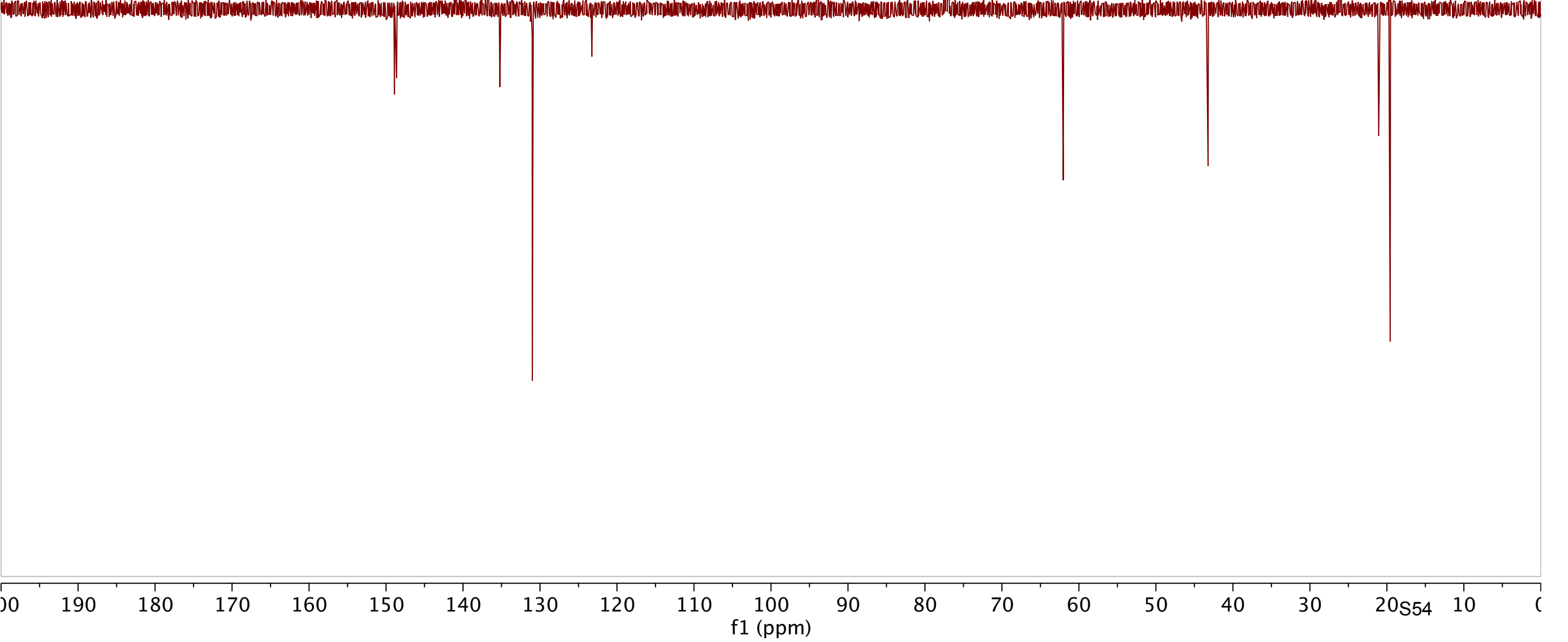




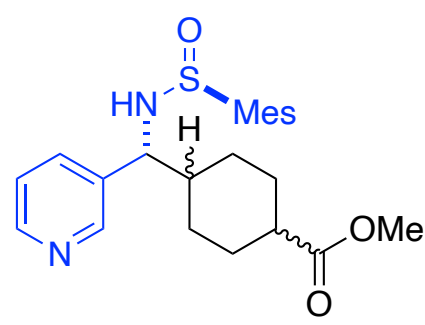

Compound 8 (2:1 trans:cis)

${ }^{1} \mathrm{H}$ NMR $\left(400 \mathrm{MHz}, \mathrm{CDCl}_{3}\right.$,

Trans and Cis not assigned

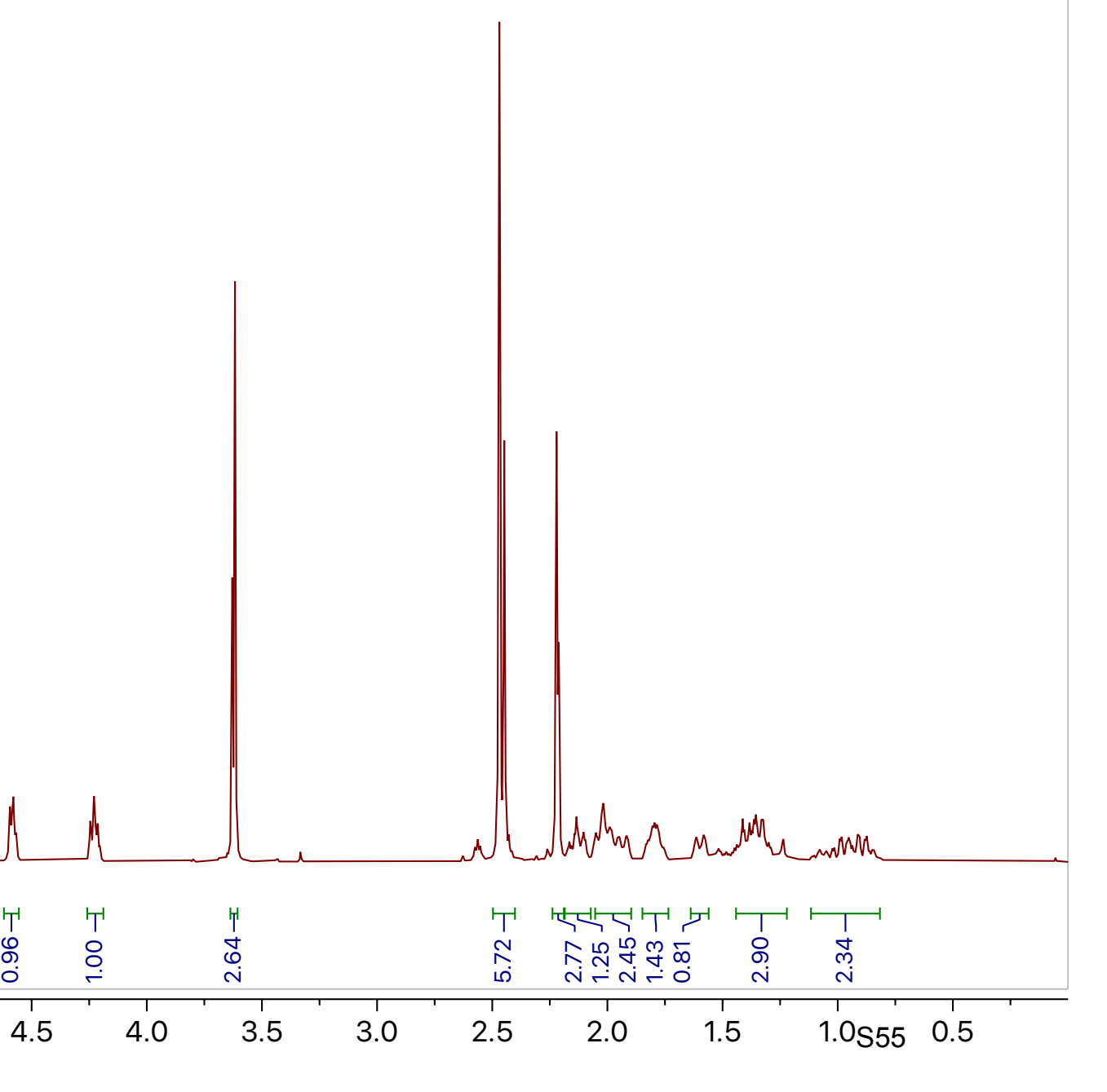




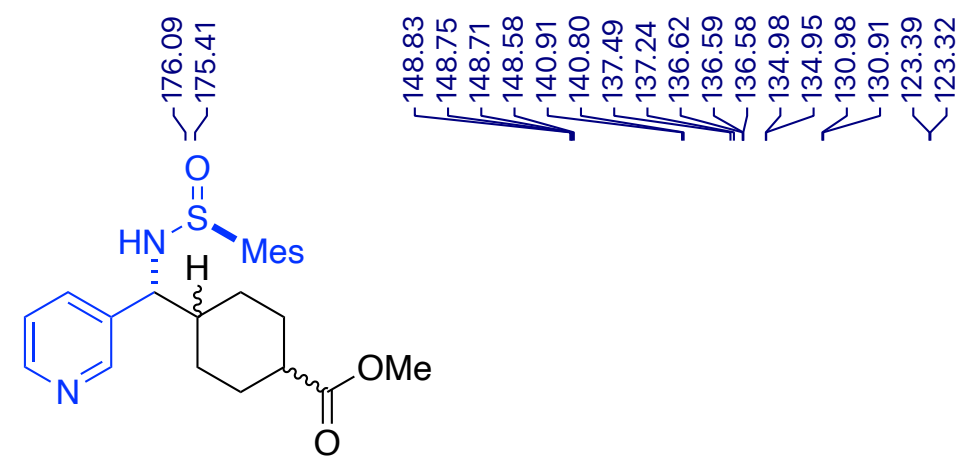

$\underbrace{0}_{1}$

Compound 8 (2:1 trans:cis)

${ }^{13} \mathrm{C}$ NMR $\left(101 \mathrm{MHz}, \mathrm{CDCl}_{3}\right.$

Trans and Cis not assigned

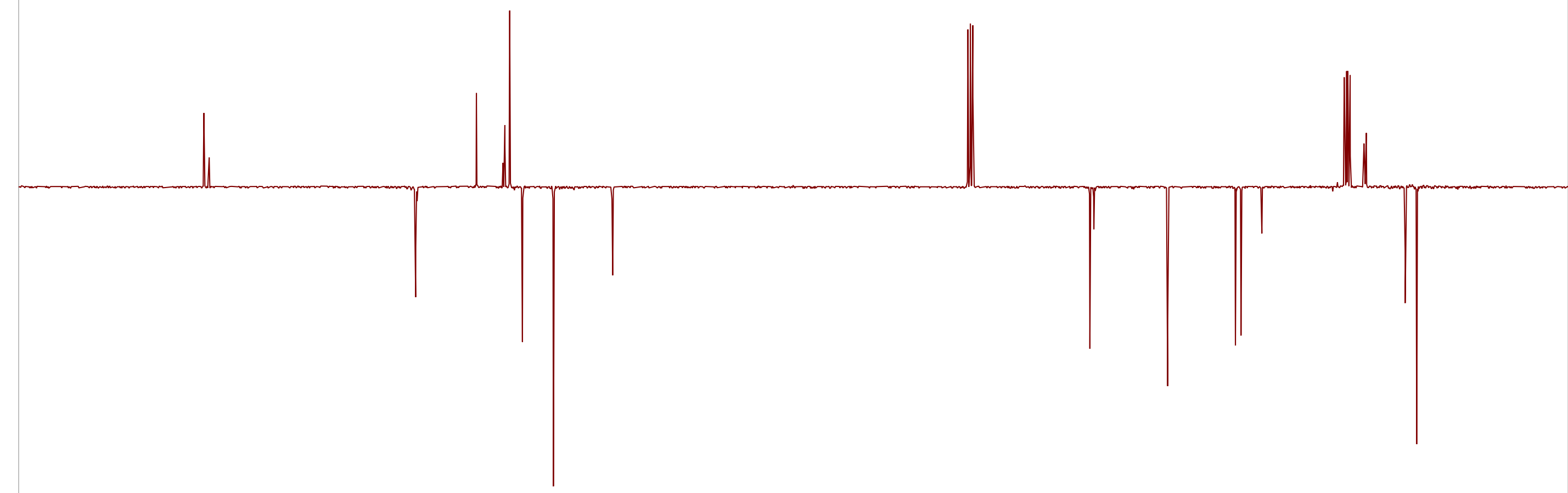

90




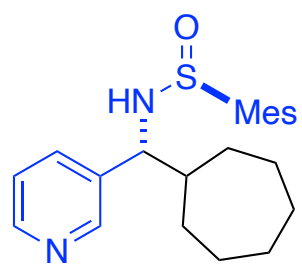

Compound 9

${ }^{1} \mathrm{H}$ NMR $\left(400 \mathrm{MHz}, \mathrm{CDCl}_{3}\right)$

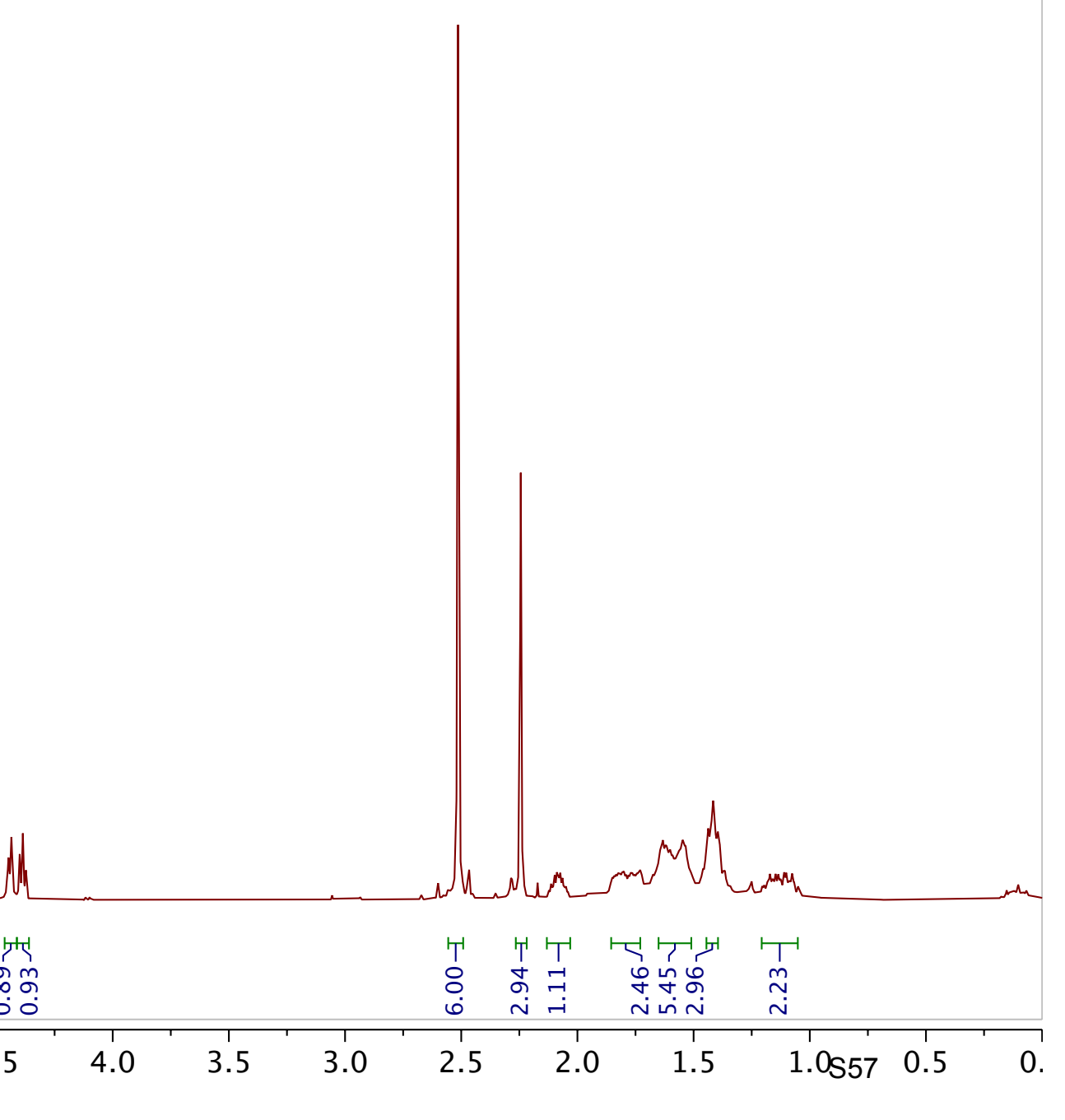




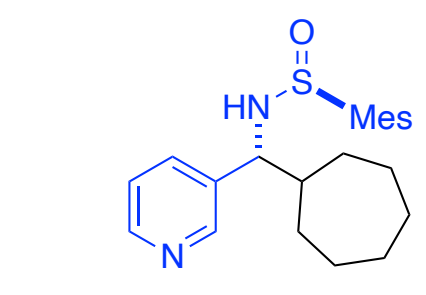

Compound 9

${ }^{13} \mathrm{C}$ NMR (101 MHz, $\left.\mathrm{CDCl}_{3}\right)$

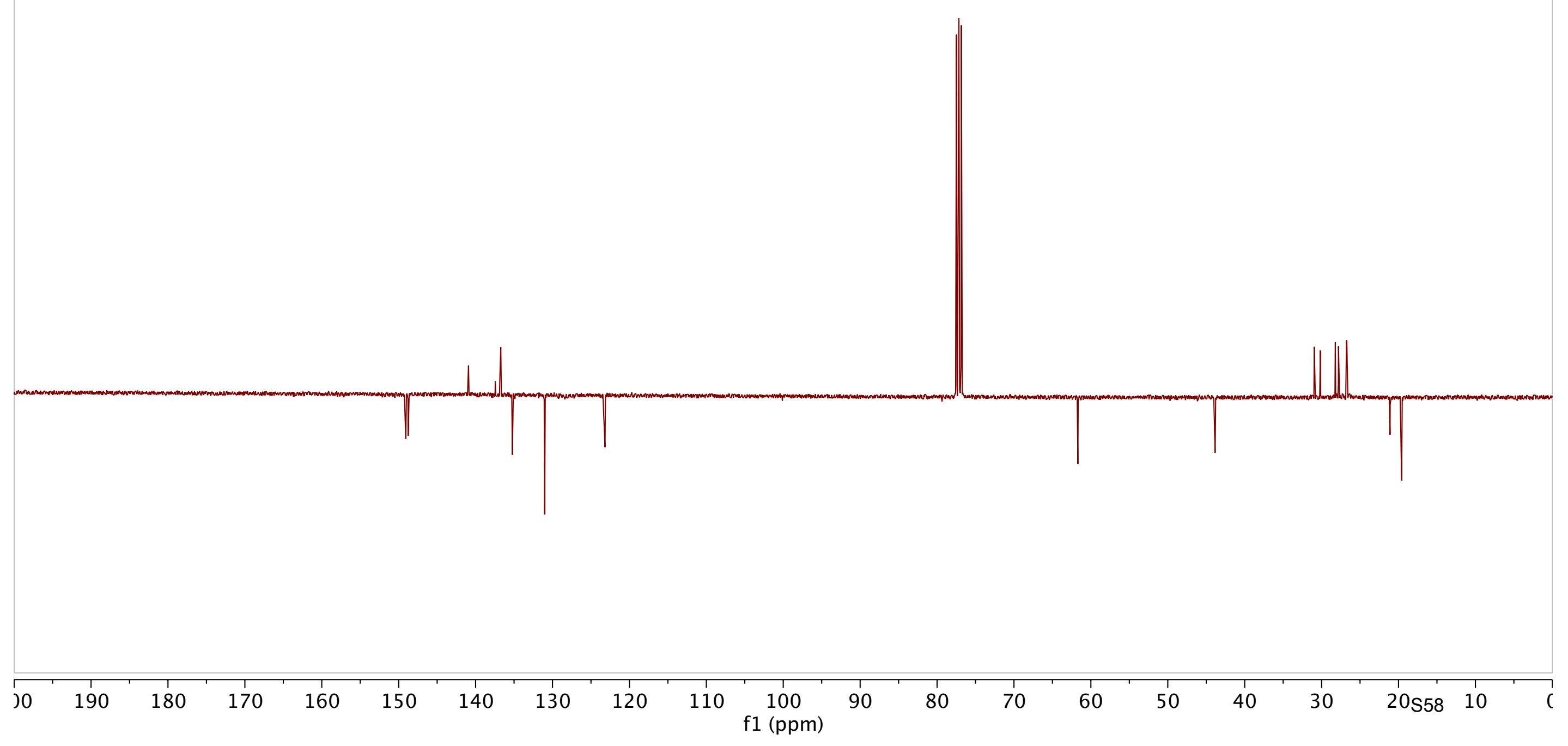




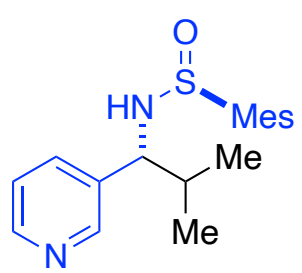

Compound 10

${ }^{1} \mathrm{H}$ NMR $\left(600 \mathrm{MHz}, \mathrm{CDCl}_{3}\right)$

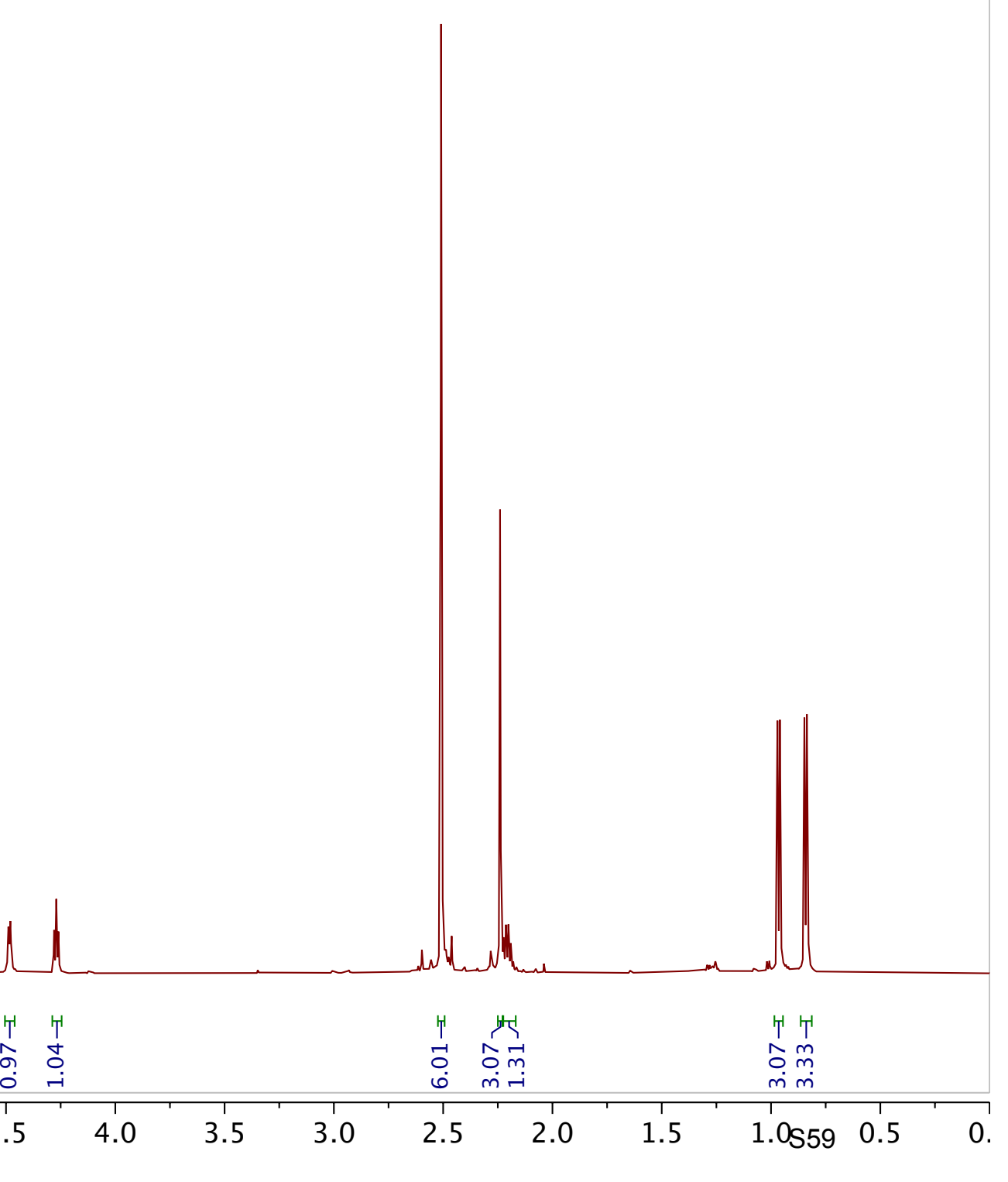




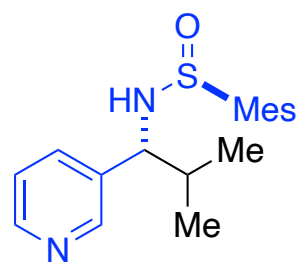

Compound 10

${ }^{13} \mathrm{C}$ NMR (151 MHz, $\mathrm{CDCl}_{3}$ )

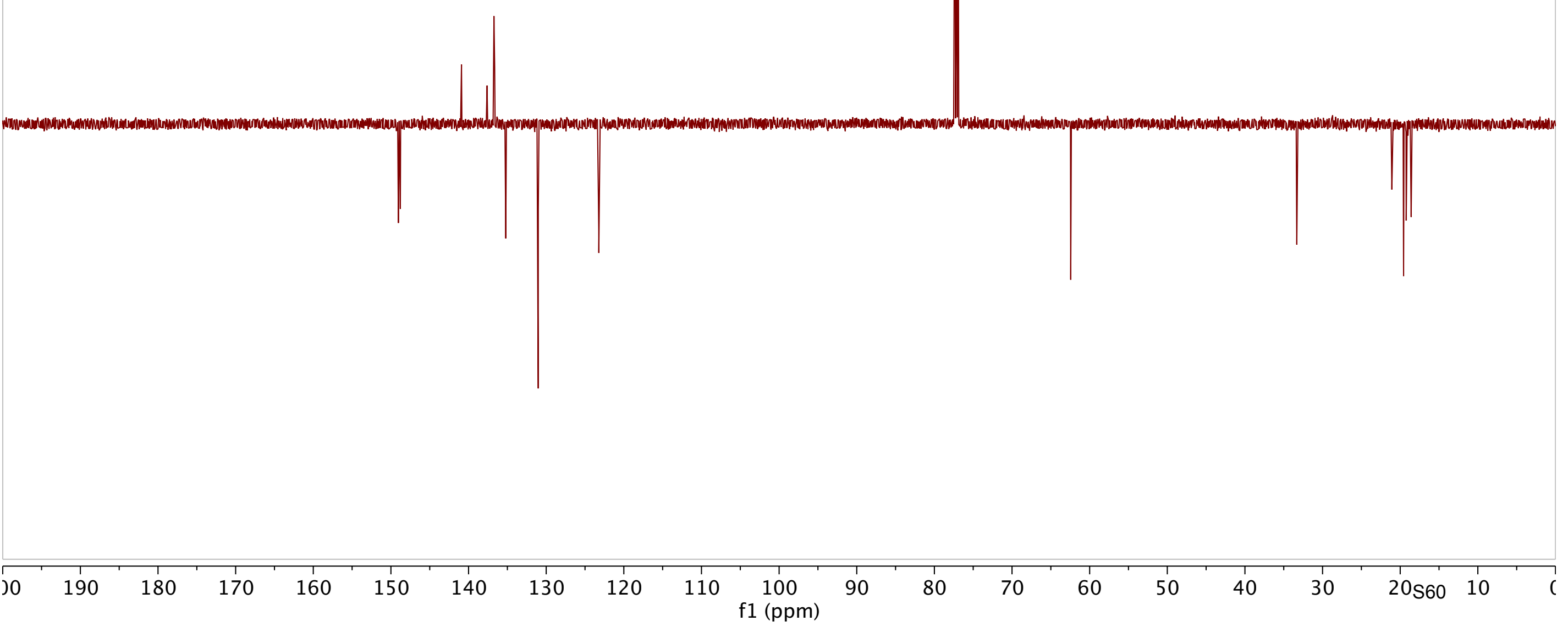


<smiles>C[C@H](CCc1ccccc1)C(NS(C)=O)c1cccnc1</smiles>

Compound 11 (1:1.1 d.r.)

${ }^{13} \mathrm{C}$ NMR $\left(101 \mathrm{MHz}, \mathrm{CDCl}_{3}\right)$

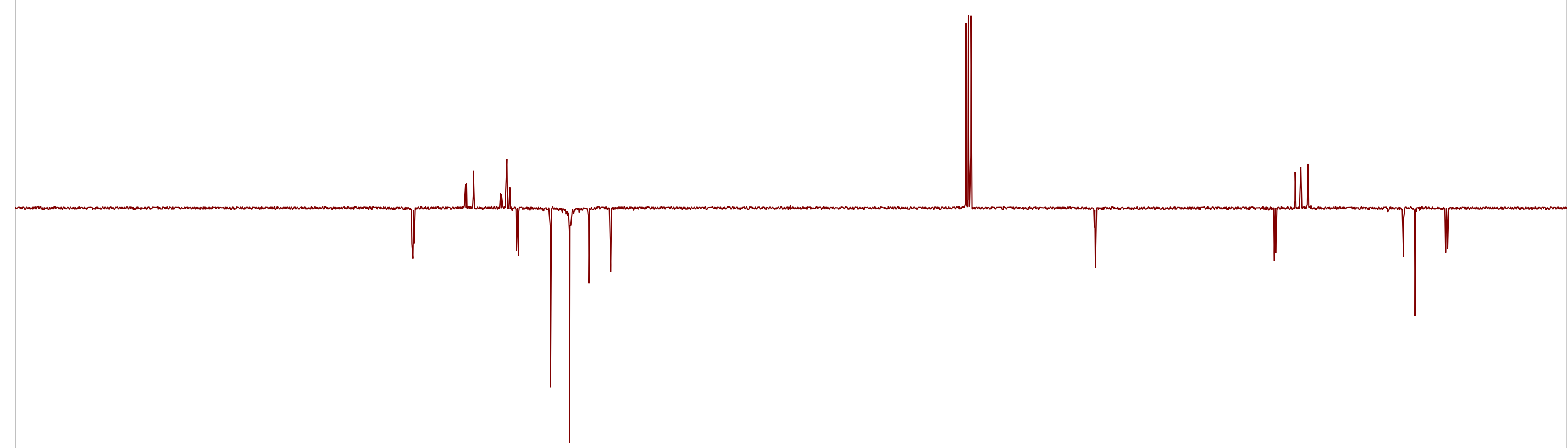




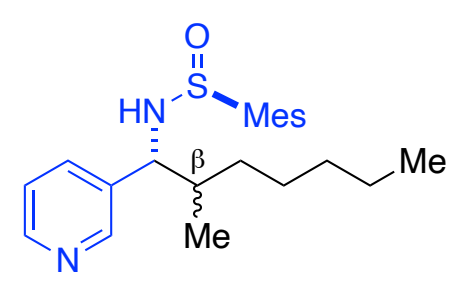

Compound 12 (1:1.2 d.r.)

${ }^{1} \mathrm{H}$ NMR $\left(600 \mathrm{MHz}, \mathrm{CDCl}_{3}\right)$

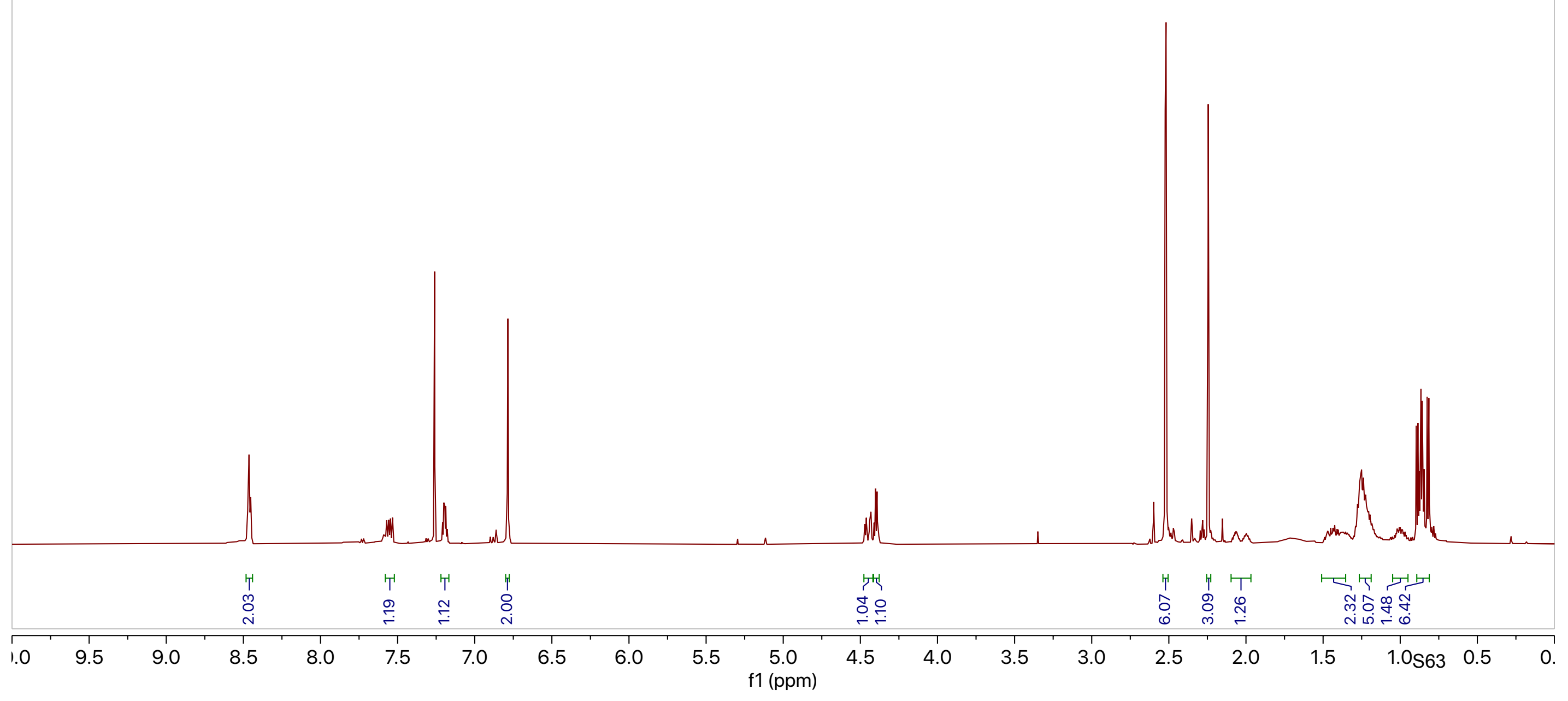




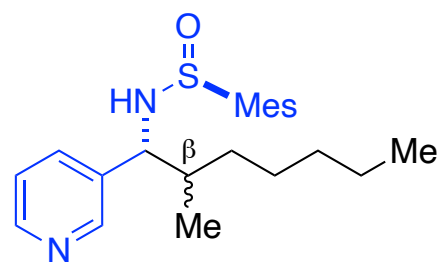

Compound 12 (1:1.2 d.r.)

${ }^{13} \mathrm{C}$ NMR $\left(151 \mathrm{MHz}, \mathrm{CDCl}_{3}\right)$

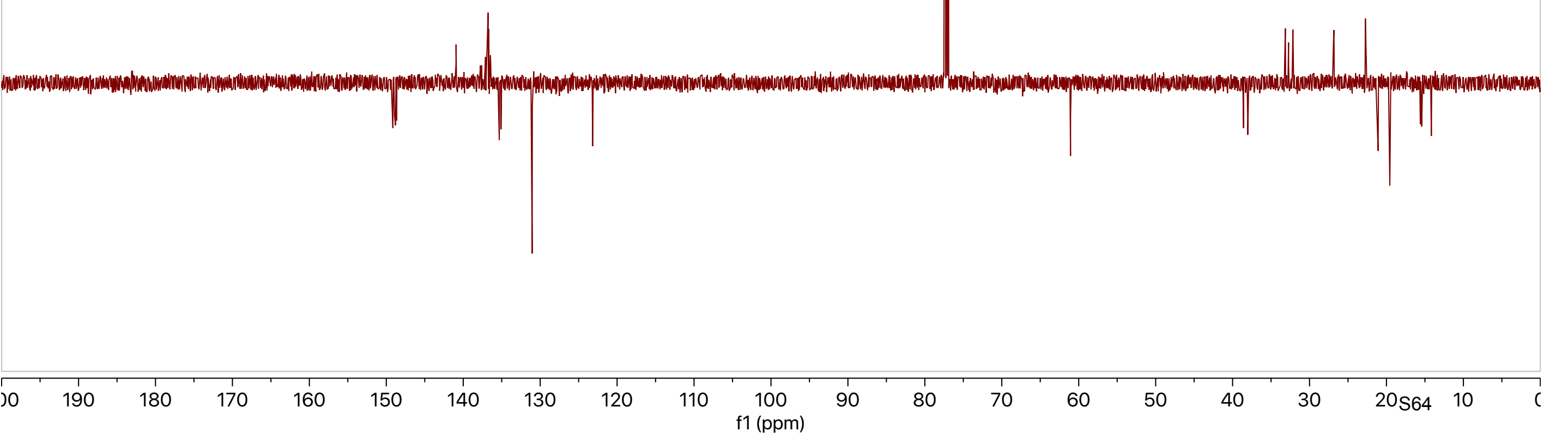




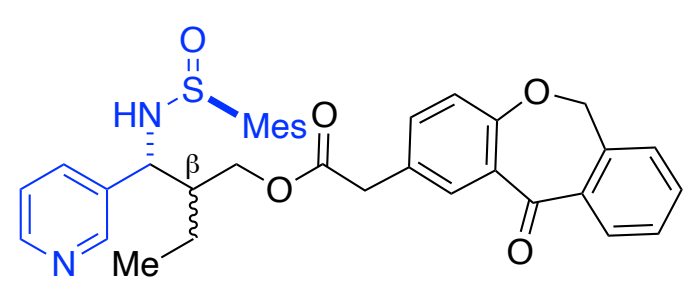

Compound $13(1: 1$ d.r.)

${ }^{1} \mathrm{H}$ NMR $\left(400 \mathrm{MHz}, \mathrm{CDCl}_{3}\right)$

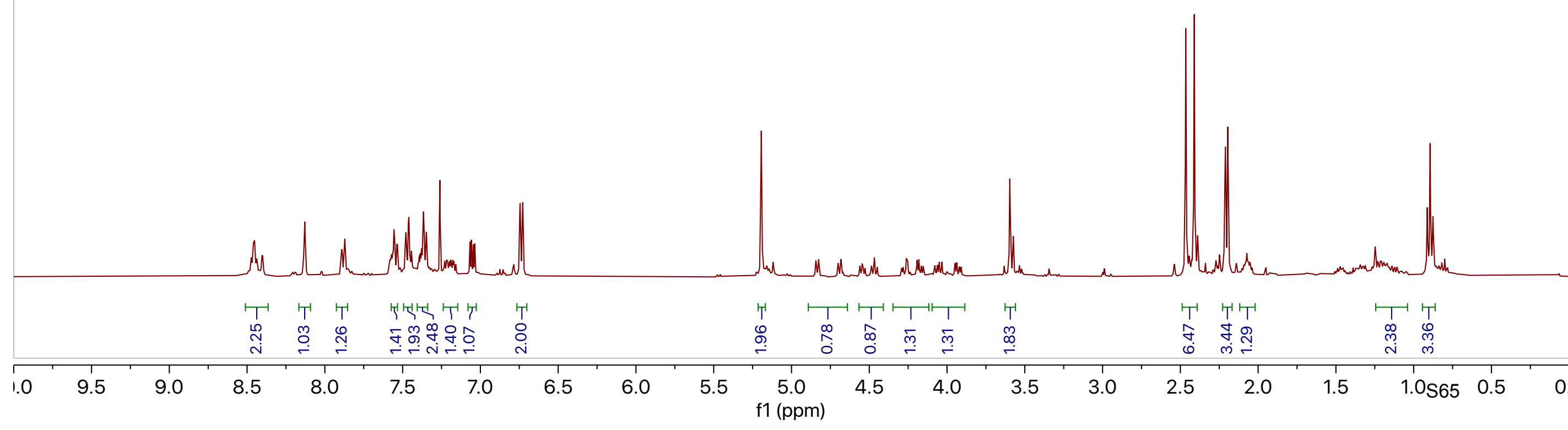




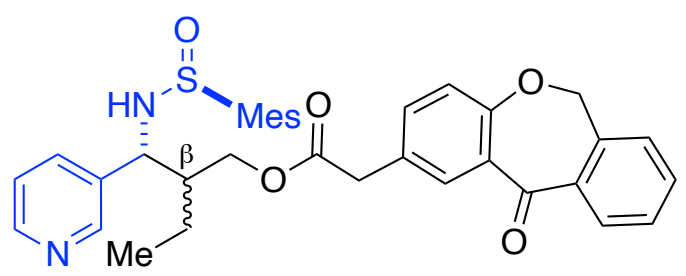

Compound 13 (1:1 d.r.)

${ }^{13} \mathrm{C} \mathrm{NMR}\left(101 \mathrm{MHz}, \mathrm{CDCl}_{3}\right)$

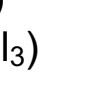




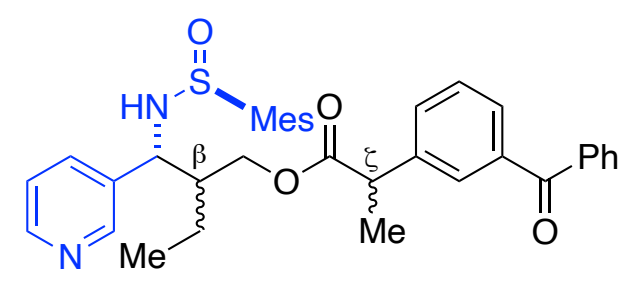

Compound $14(1: 1: 1: 1$ d.r.)

${ }^{1} \mathrm{H}$ NMR $\left(400 \mathrm{MHz}, \mathrm{CDCl}_{3}\right)$

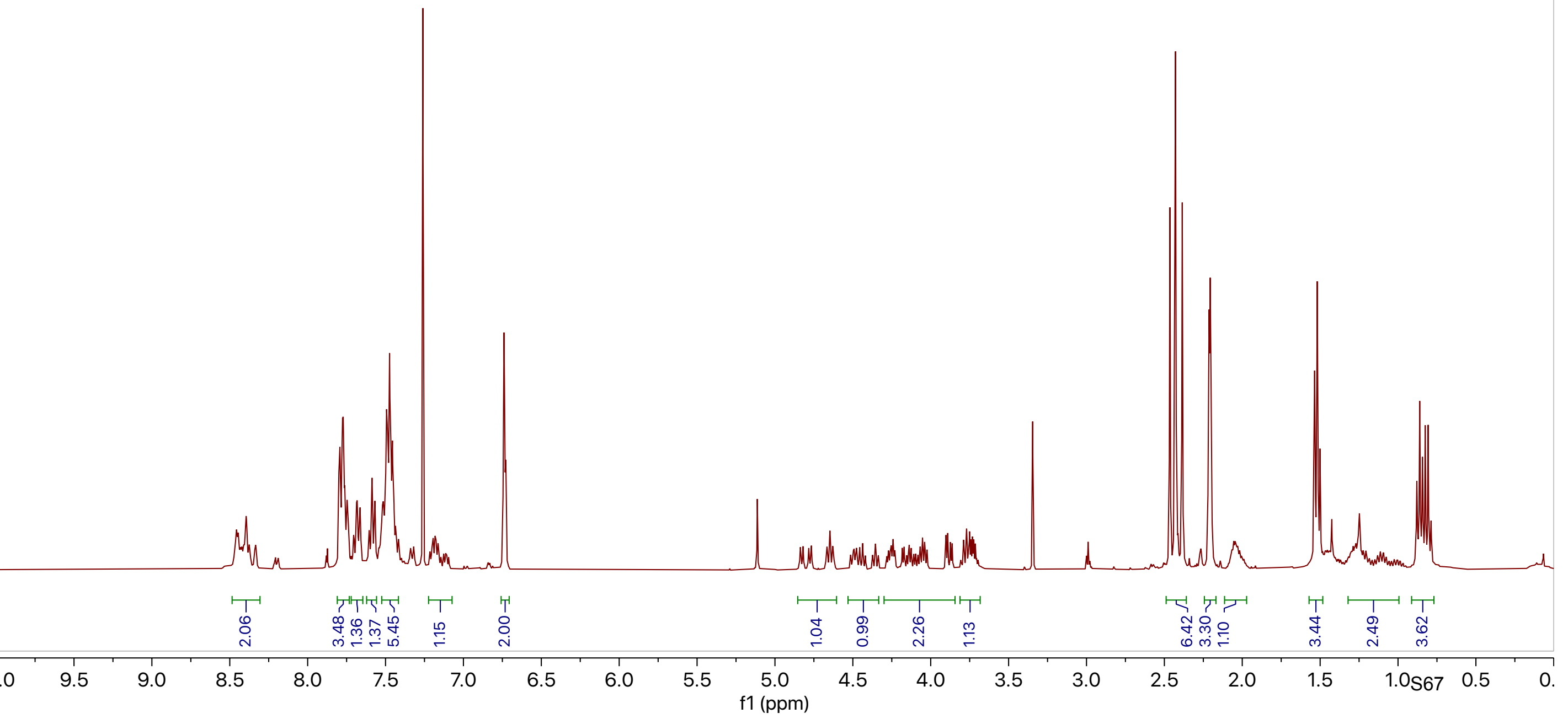




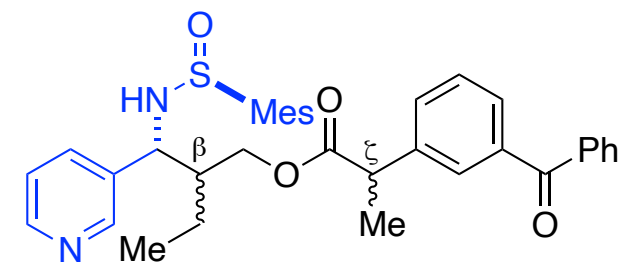

Compound $14(1: 1: 1: 1$ d.r. $)$

${ }^{13} \mathrm{C}$ NMR $\left(101 \mathrm{MHz}, \mathrm{CDCl}_{3}\right)$

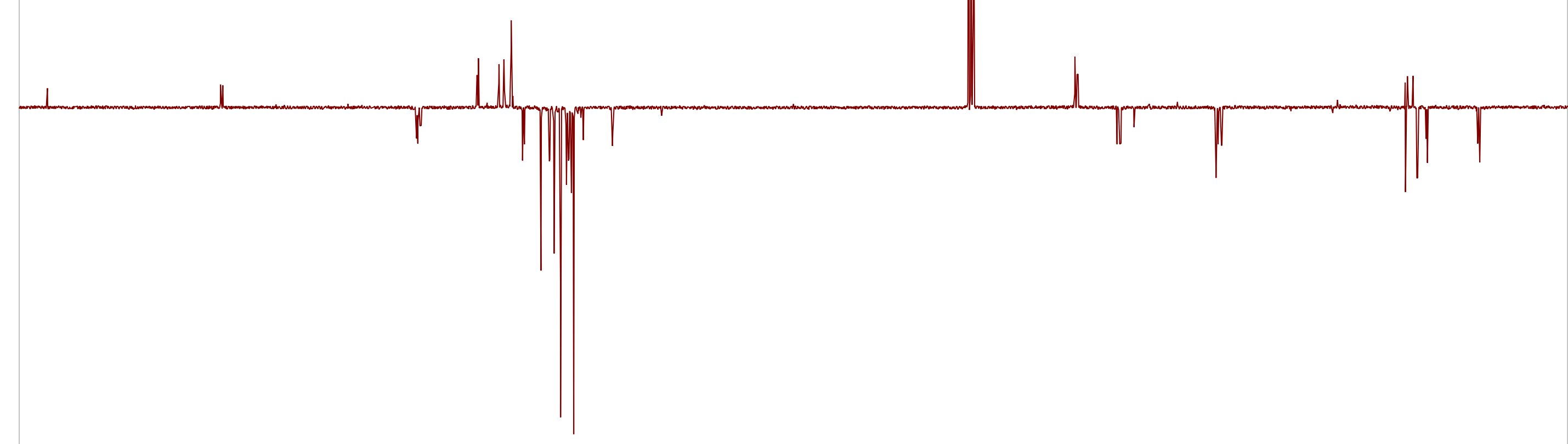

\begin{tabular}{|c|c|c|c|c|c|c|c|c|c|c|c|c|c|c|c|c|c|c|c|}
\hline )0 & 190 & 180 & 170 & 160 & 150 & 140 & 130 & 120 & 110 & $\begin{array}{c}100 \\
\mathrm{f} 1(\mathrm{ppm})\end{array}$ & 90 & 80 & 70 & 60 & 50 & 40 & 30 & $20 S 68 \quad 10$ & $c$ \\
\hline
\end{tabular}




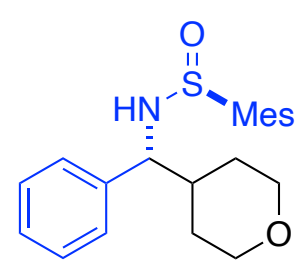

Compound 15

${ }^{1} \mathrm{H}$ NMR $\left(600 \mathrm{MHz}, \mathrm{CDCl}_{3}\right)$

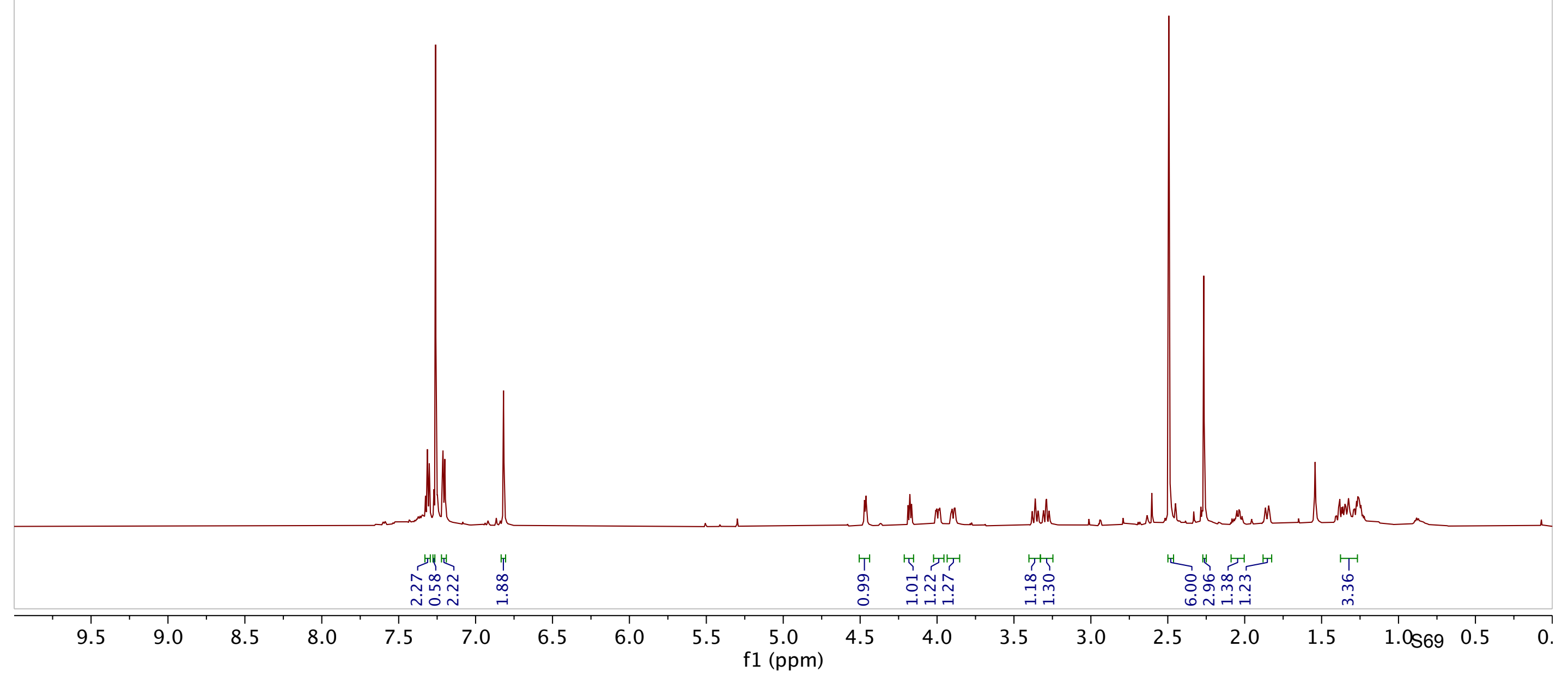




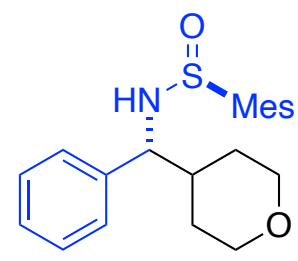

Compound 15

${ }^{13} \mathrm{C}$ NMR (151 MHz, $\mathrm{CDCl}_{3}$ )

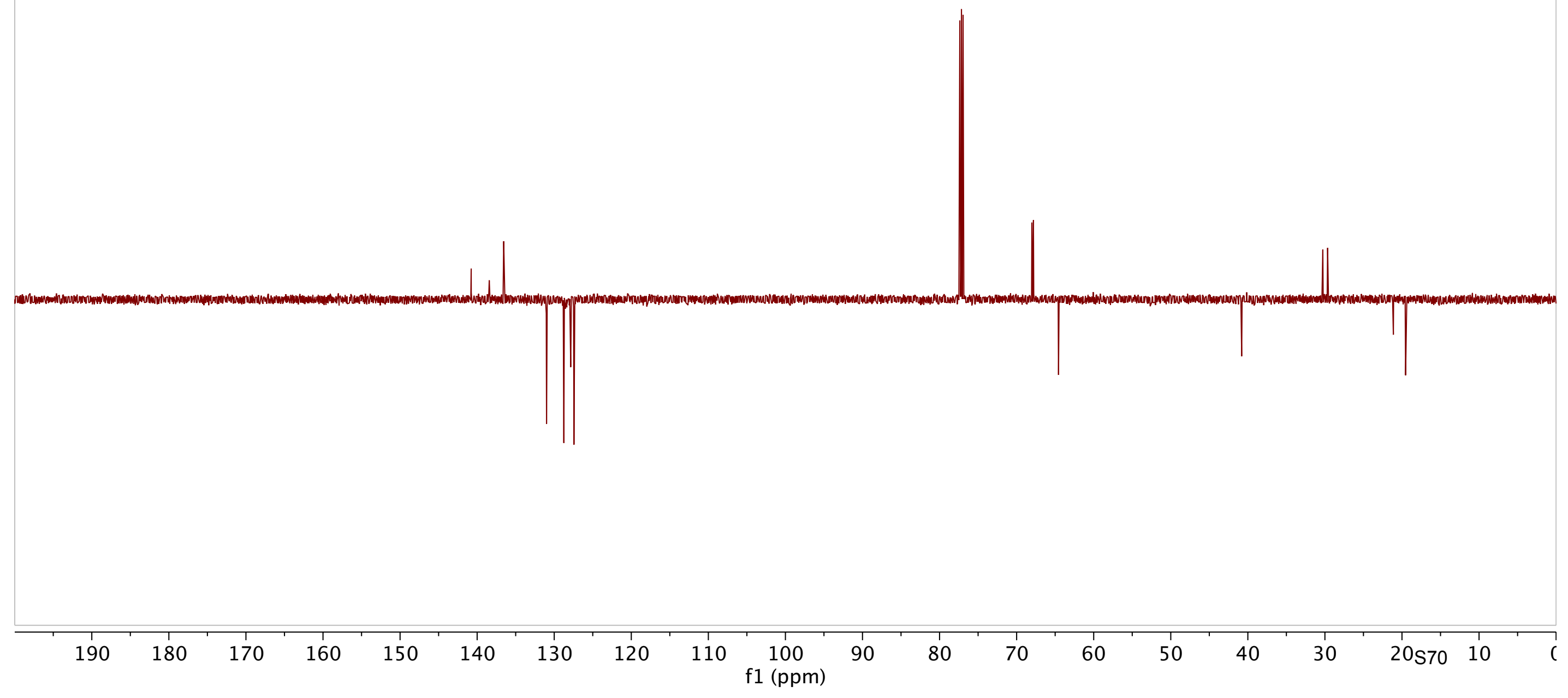




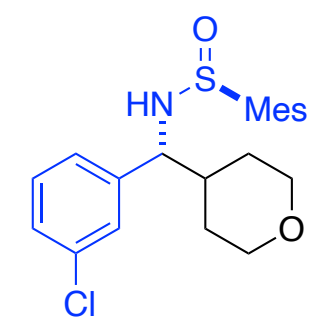

Compound 16

${ }^{1} \mathrm{H}$ NMR $\left(600 \mathrm{MHz}, \mathrm{CDCl}_{3}\right)$

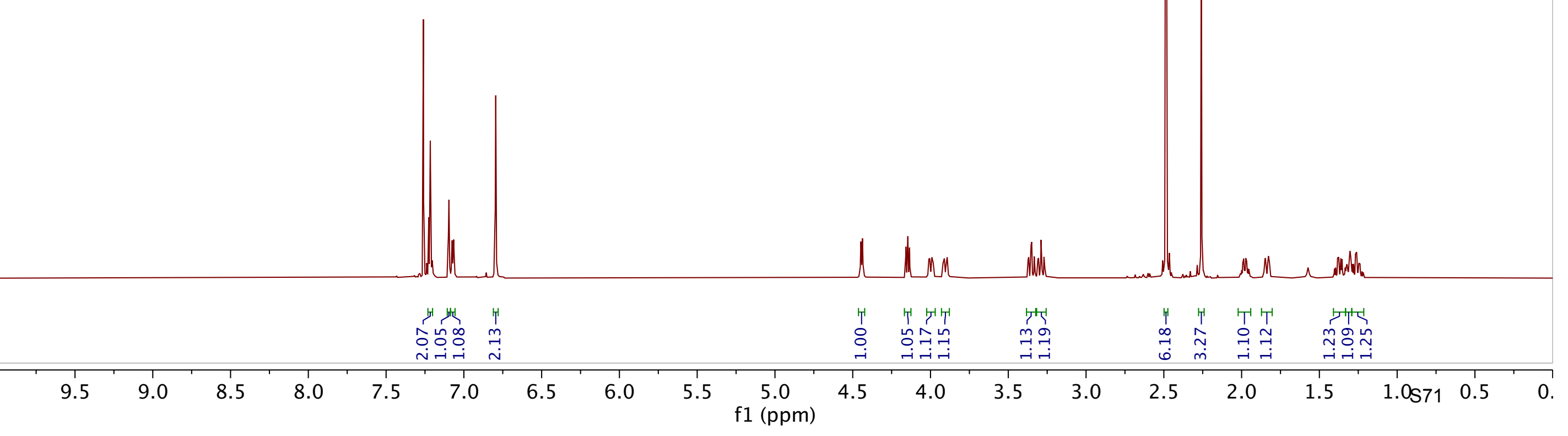




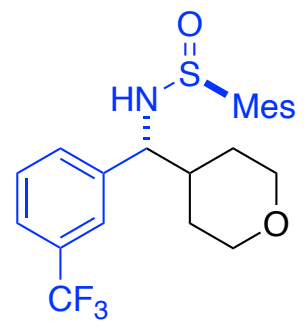

Compound 17

${ }^{1} \mathrm{H} \mathrm{NMR}\left(400 \mathrm{MHz}, \mathrm{CDCl}_{3}\right)$

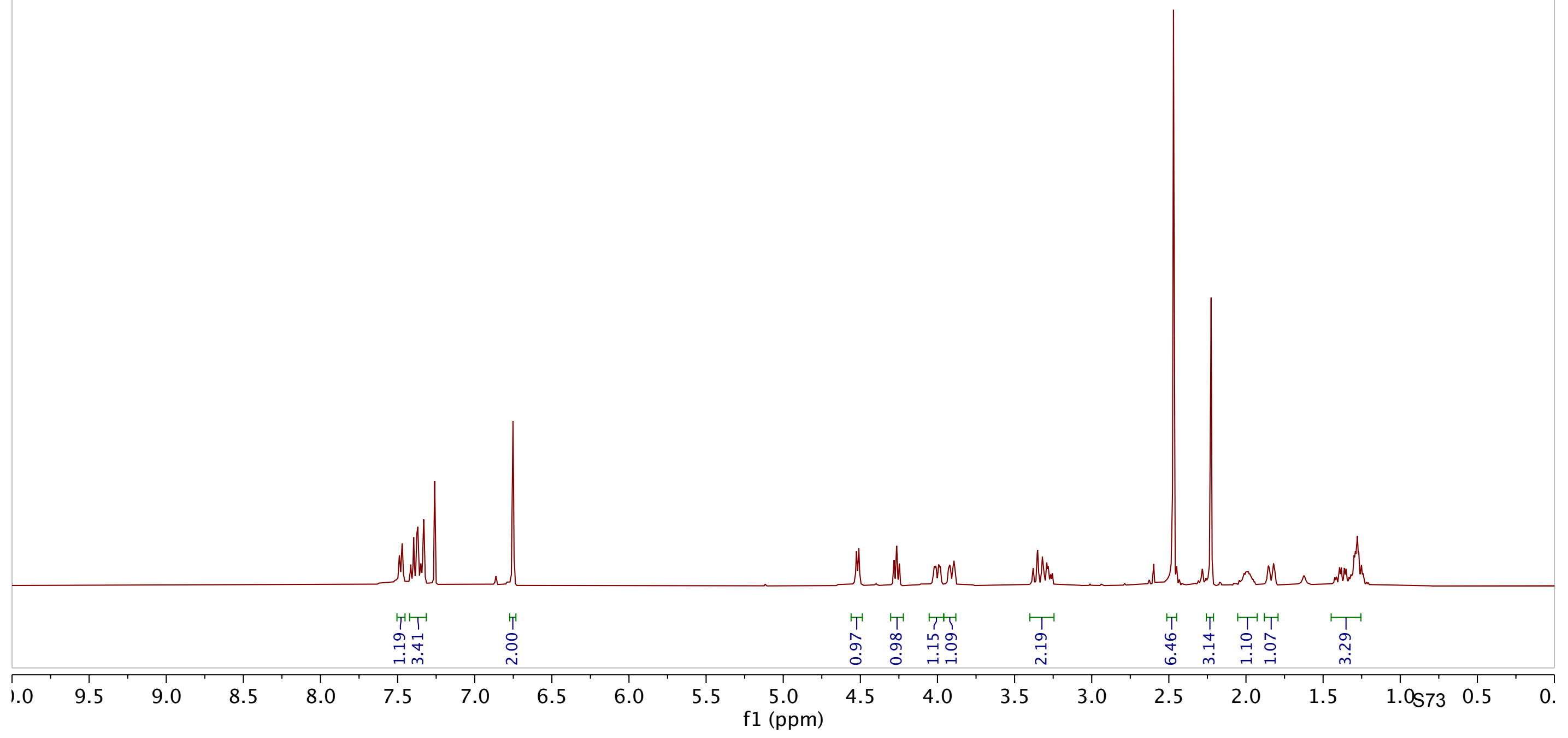




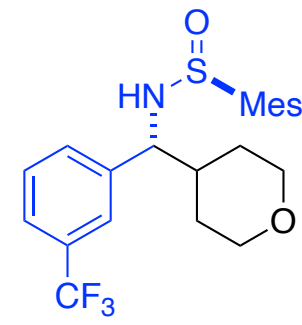

Compound 17

${ }^{13} \mathrm{C}$ NMR (101 MHz, $\left.\mathrm{CDCl}_{3}\right)$

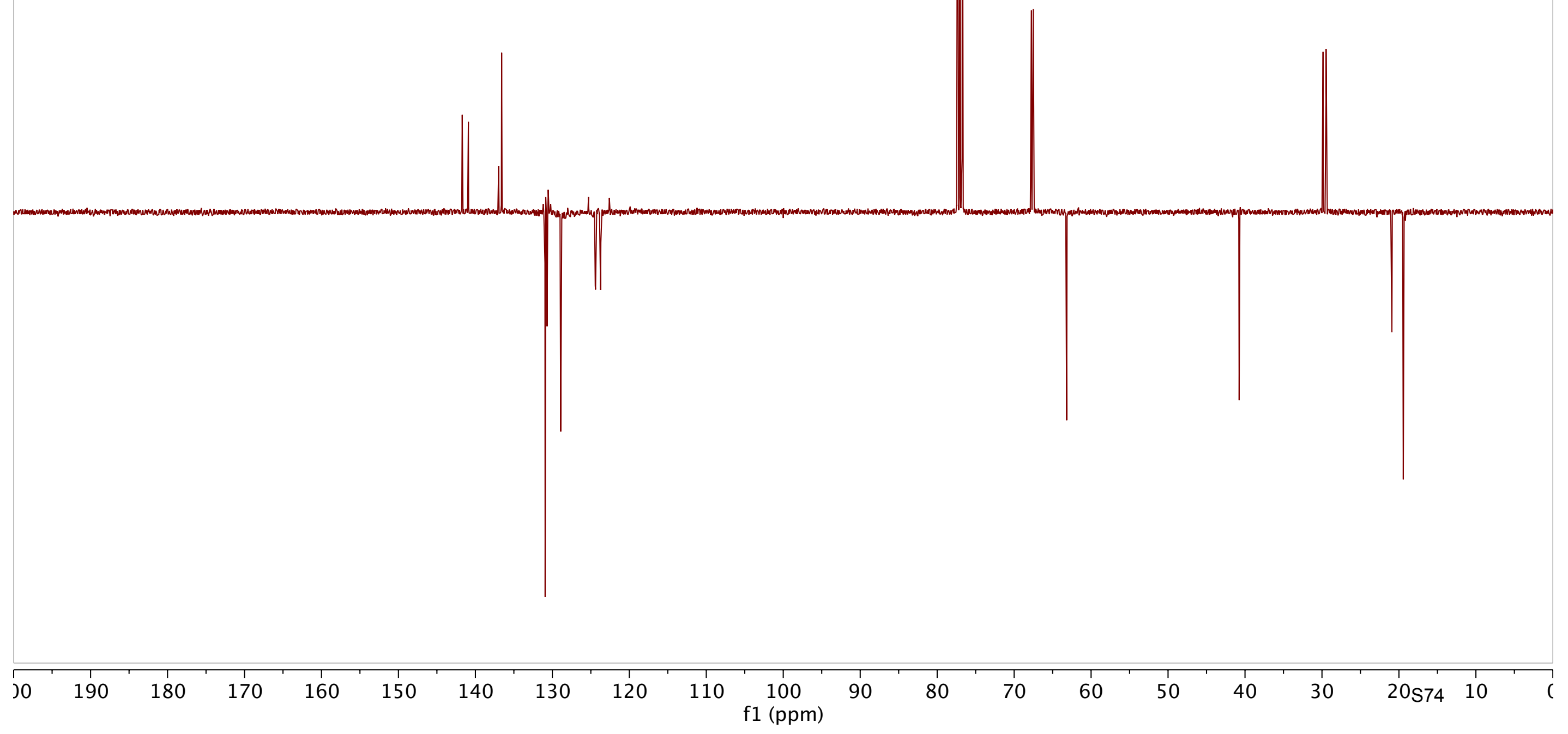




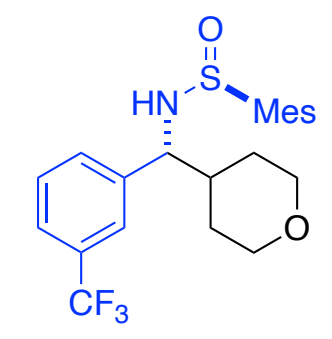

Compound 17

${ }^{19} \mathrm{~F} \mathrm{NMR}\left(376 \mathrm{MHz}, \mathrm{CDCl}_{3}\right)$

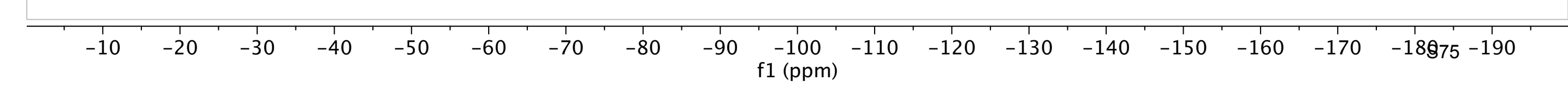




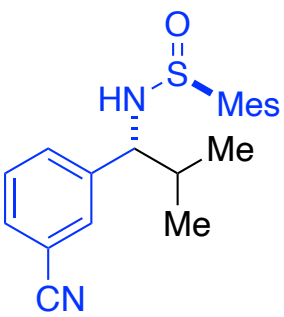

Compound 18

${ }^{1} \mathrm{H}$ NMR $\left(600 \mathrm{MHz}, \mathrm{CDCl}_{3}\right)$ 


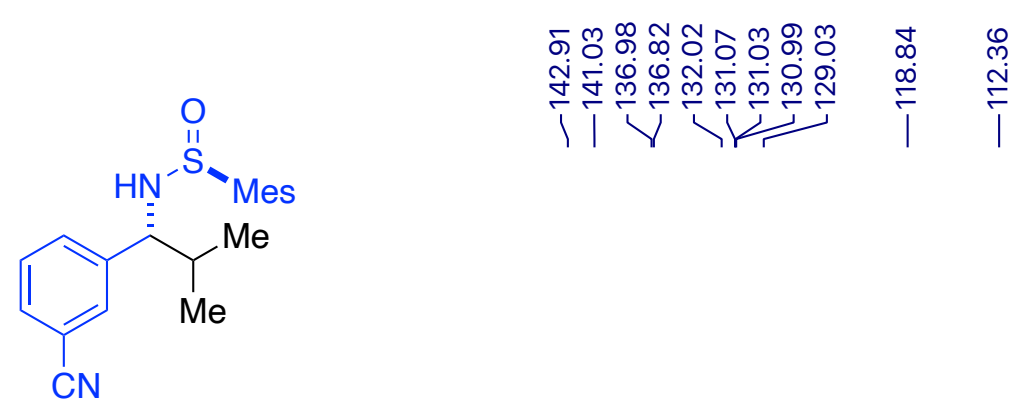

Compound 18

${ }^{13} \mathrm{C}$ NMR (151 MHz, $\mathrm{CDCl}_{3}$ )

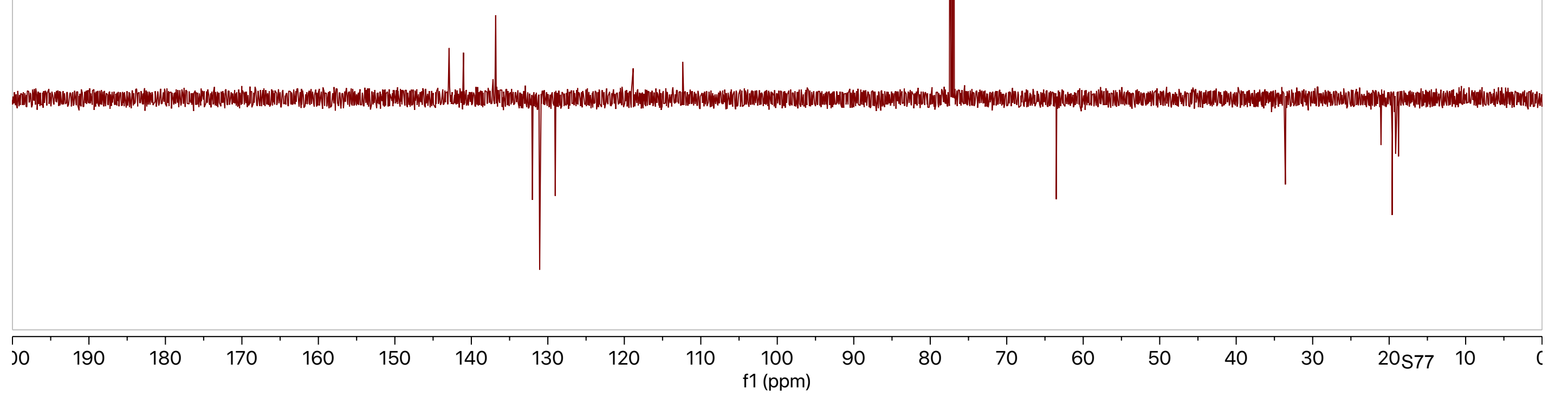




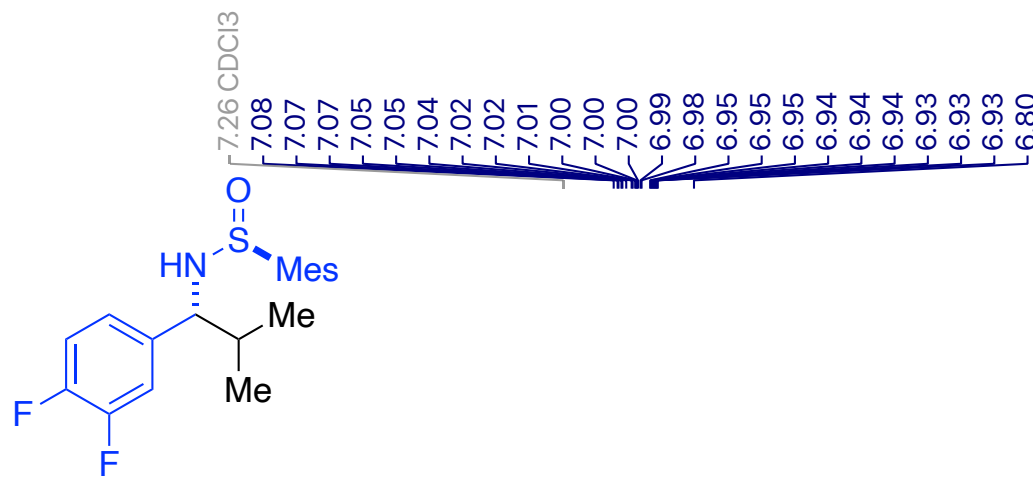

\section{Compound 19}

${ }^{1} \mathrm{H}$ NMR $\left(600 \mathrm{MHz}, \mathrm{CDCl}_{3}\right)$

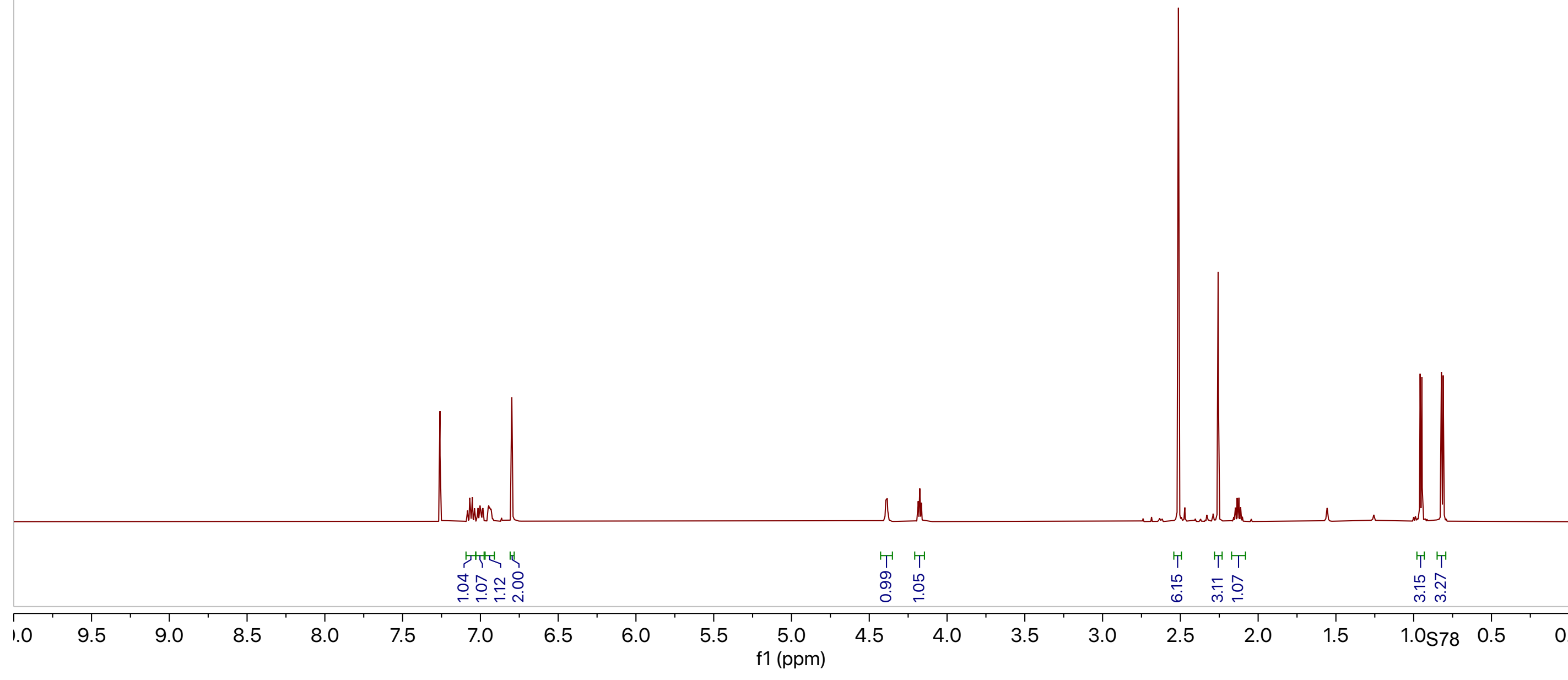




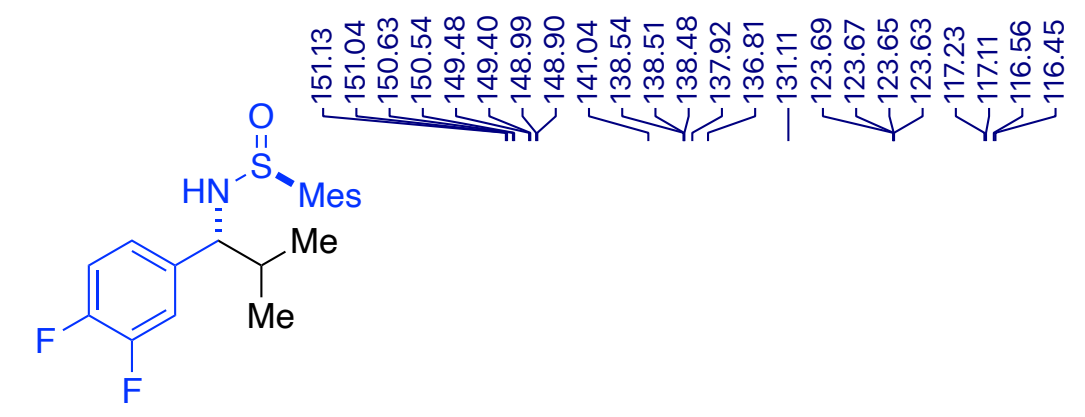

Compound 19

${ }^{13} \mathrm{C}$ NMR (151 MHz, $\mathrm{CDCl}_{3}$ )

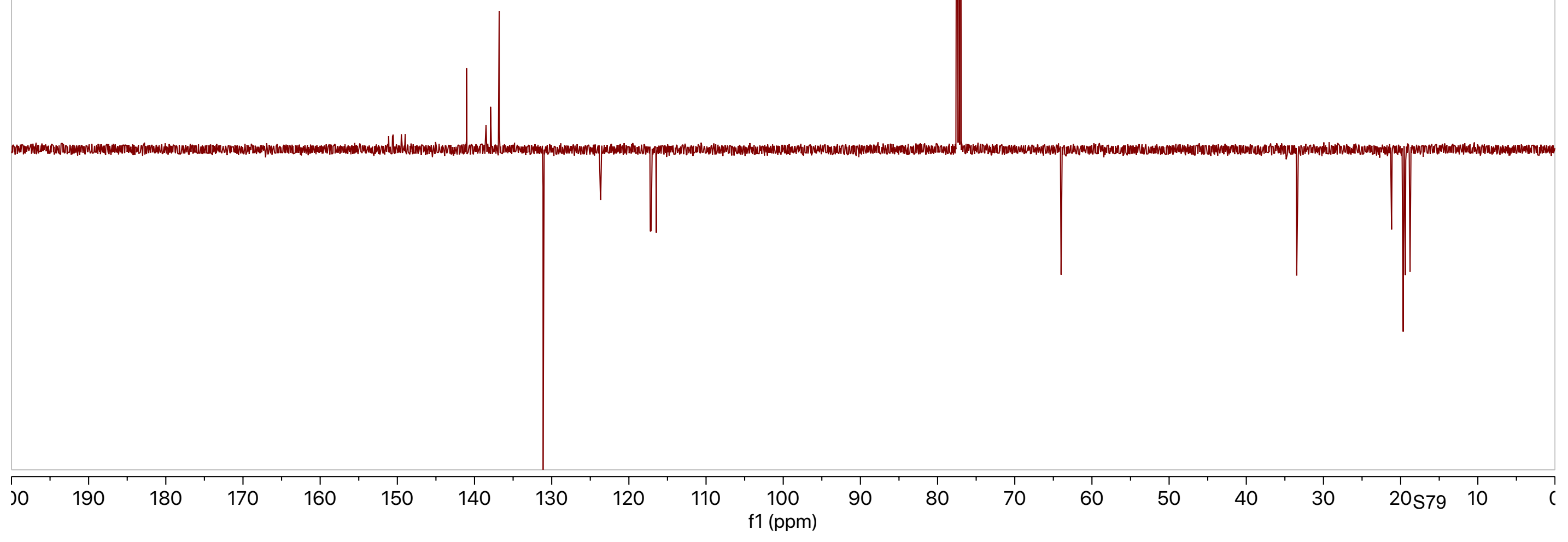




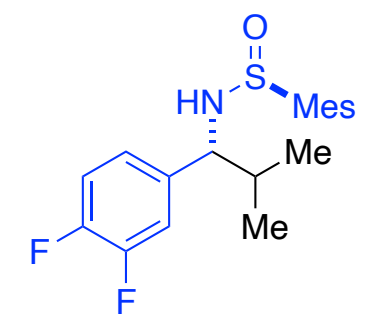

ำ ำ ๆ ?

लें लें

نं

Compound 19

${ }^{19} \mathrm{~F} \mathrm{NMR} \mathrm{(565} \mathrm{MHz,} \mathrm{CDCl}_{3}$ )

Lu||




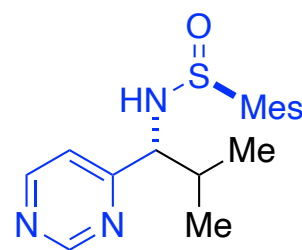

Compound 20

${ }^{1} \mathrm{H}$ NMR $\left(600 \mathrm{MHz}, \mathrm{CDCl}_{3}\right)$

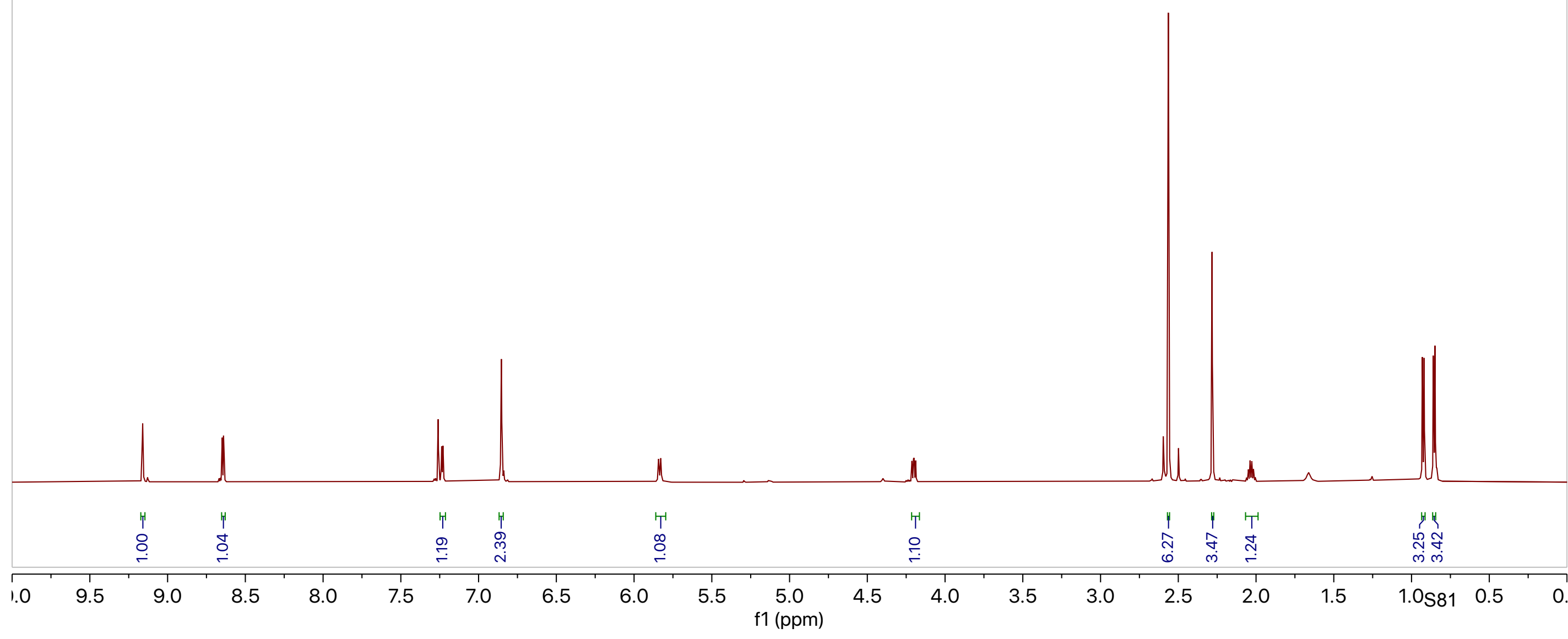




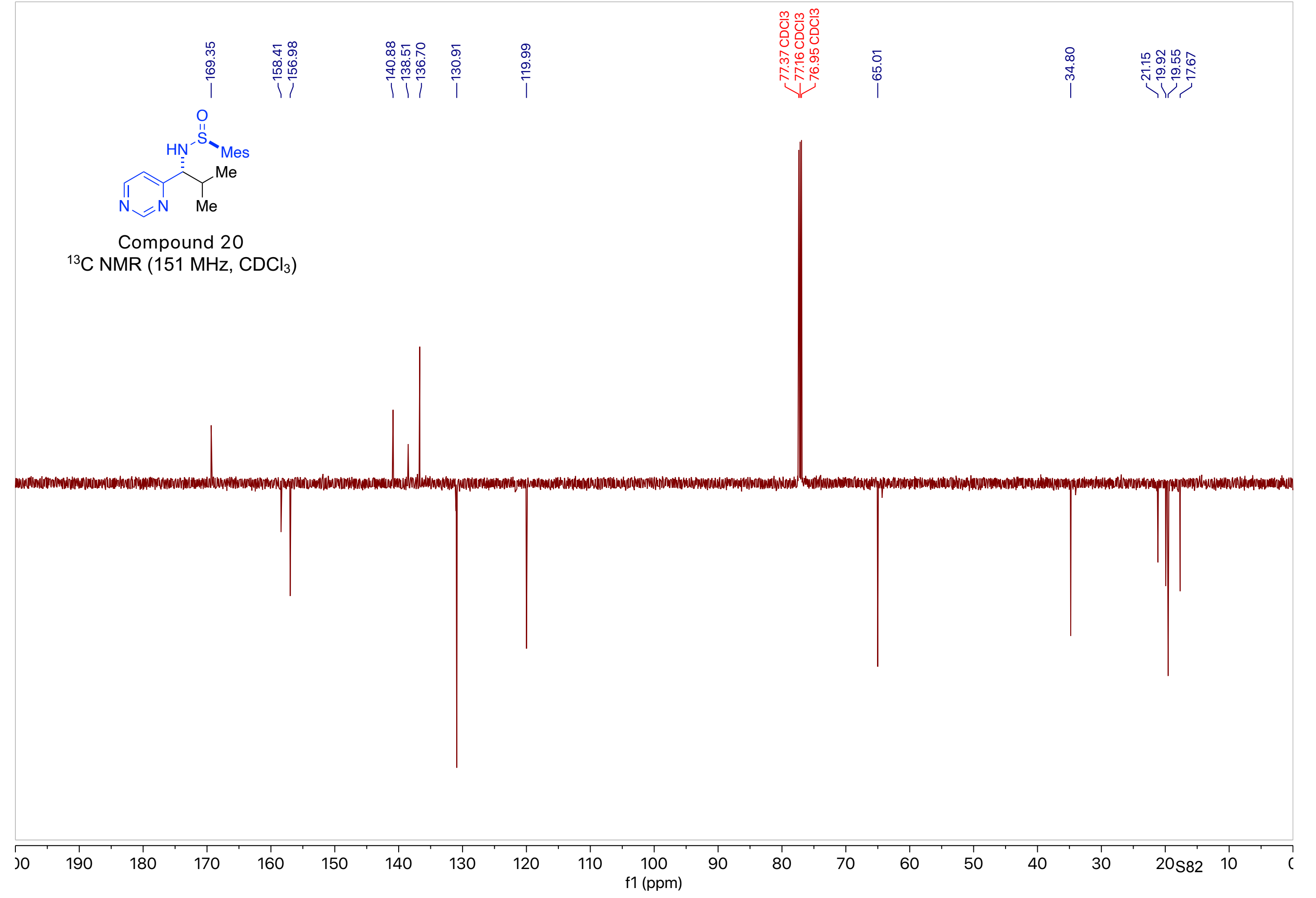




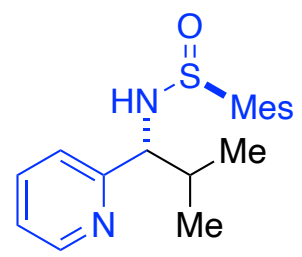

Compound 21

${ }^{1} \mathrm{H} \mathrm{NMR}\left(600 \mathrm{MHz}, \mathrm{CDCl}_{3}\right)$
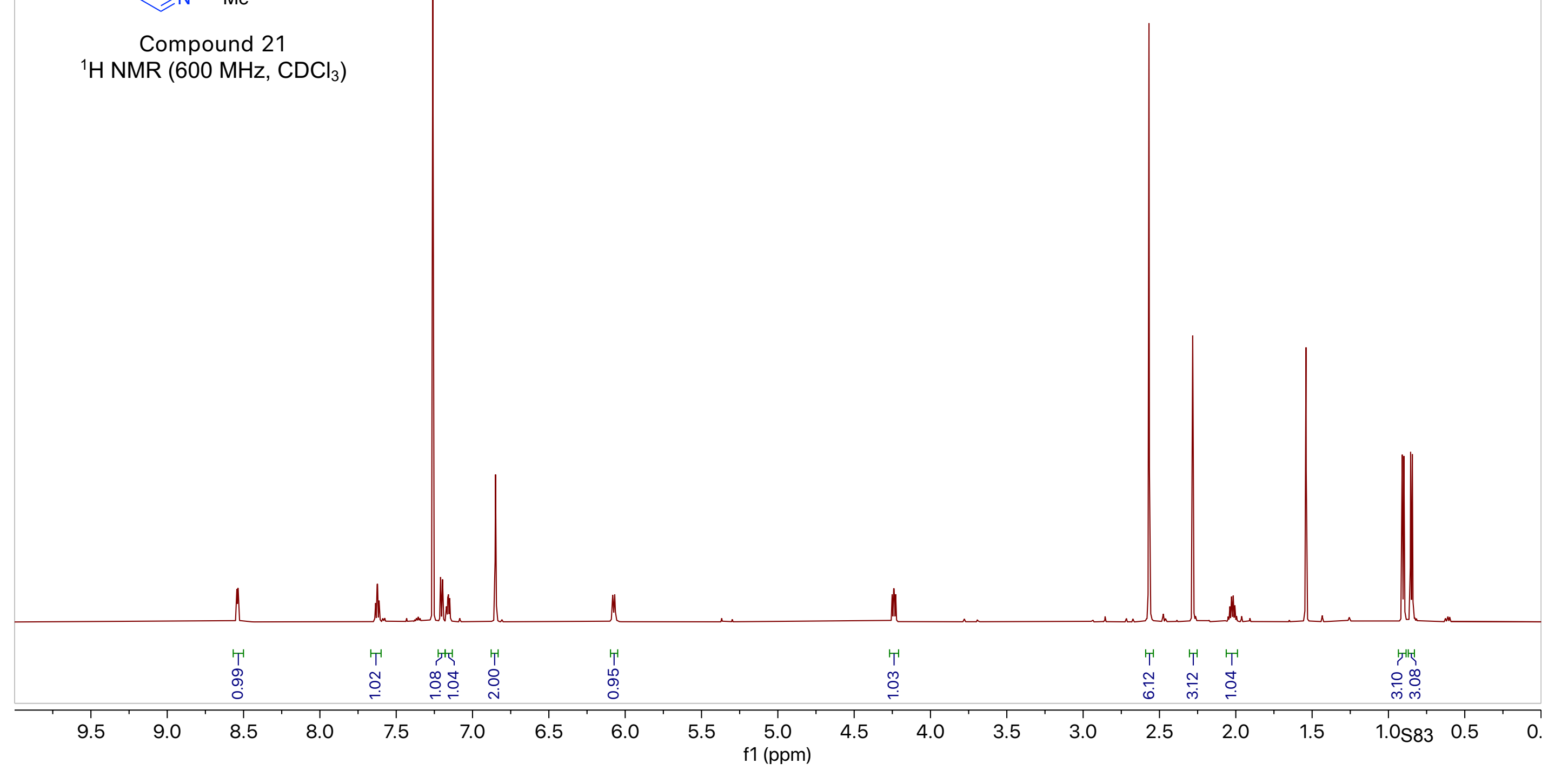


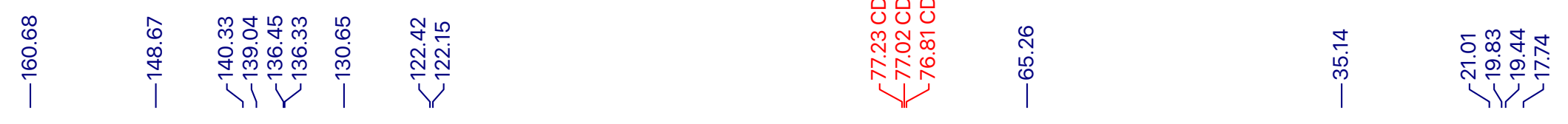

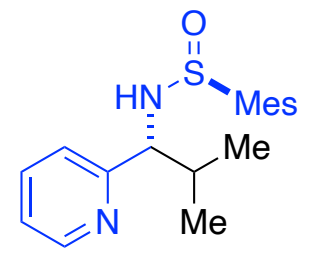

Compound 21

${ }^{13} \mathrm{C}$ NMR $\left(151 \mathrm{MHz}, \mathrm{CDCl}_{3}\right)$

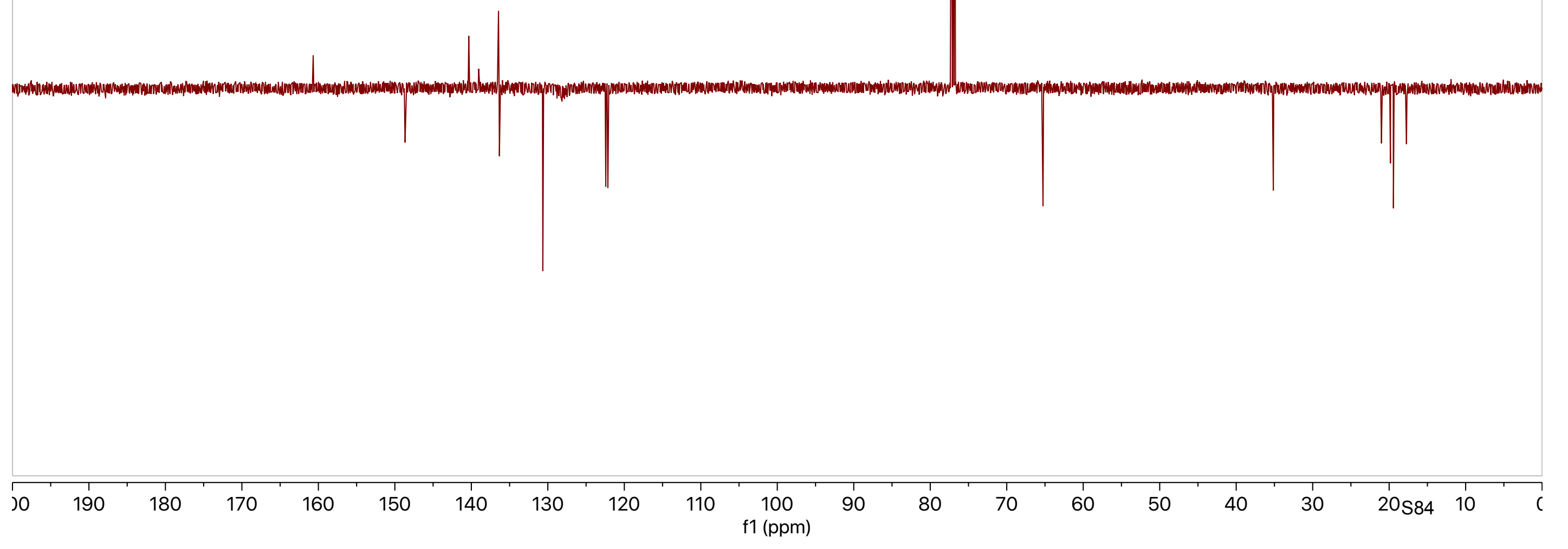




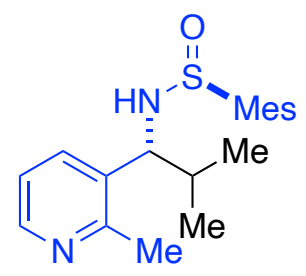

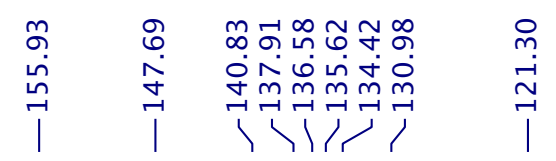

Compound 22

${ }^{13} \mathrm{C}$ NMR (101 MHz, $\mathrm{CDCl}_{3}$ )

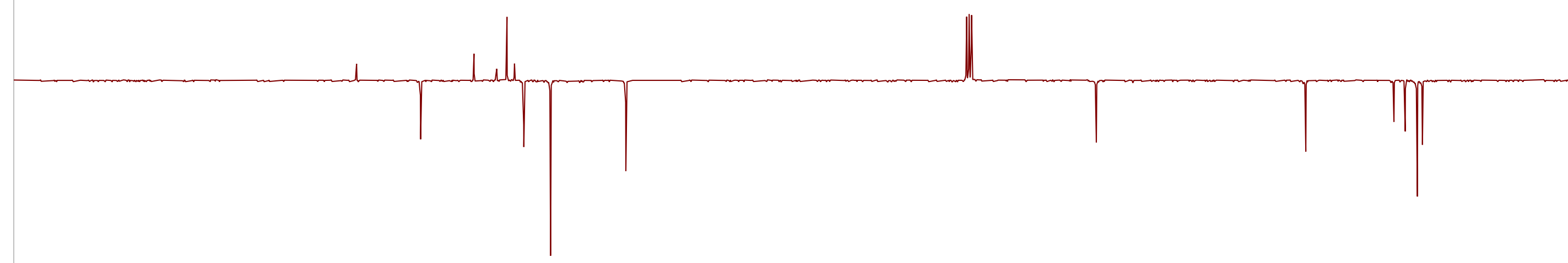




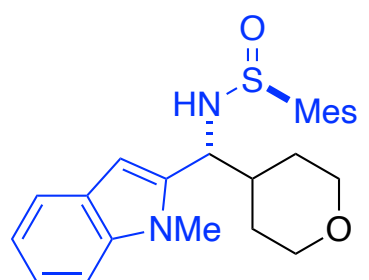

Compound 23

${ }^{1} \mathrm{H}$ NMR $\left(400 \mathrm{MHz}, \mathrm{CDCl}_{3}\right)$

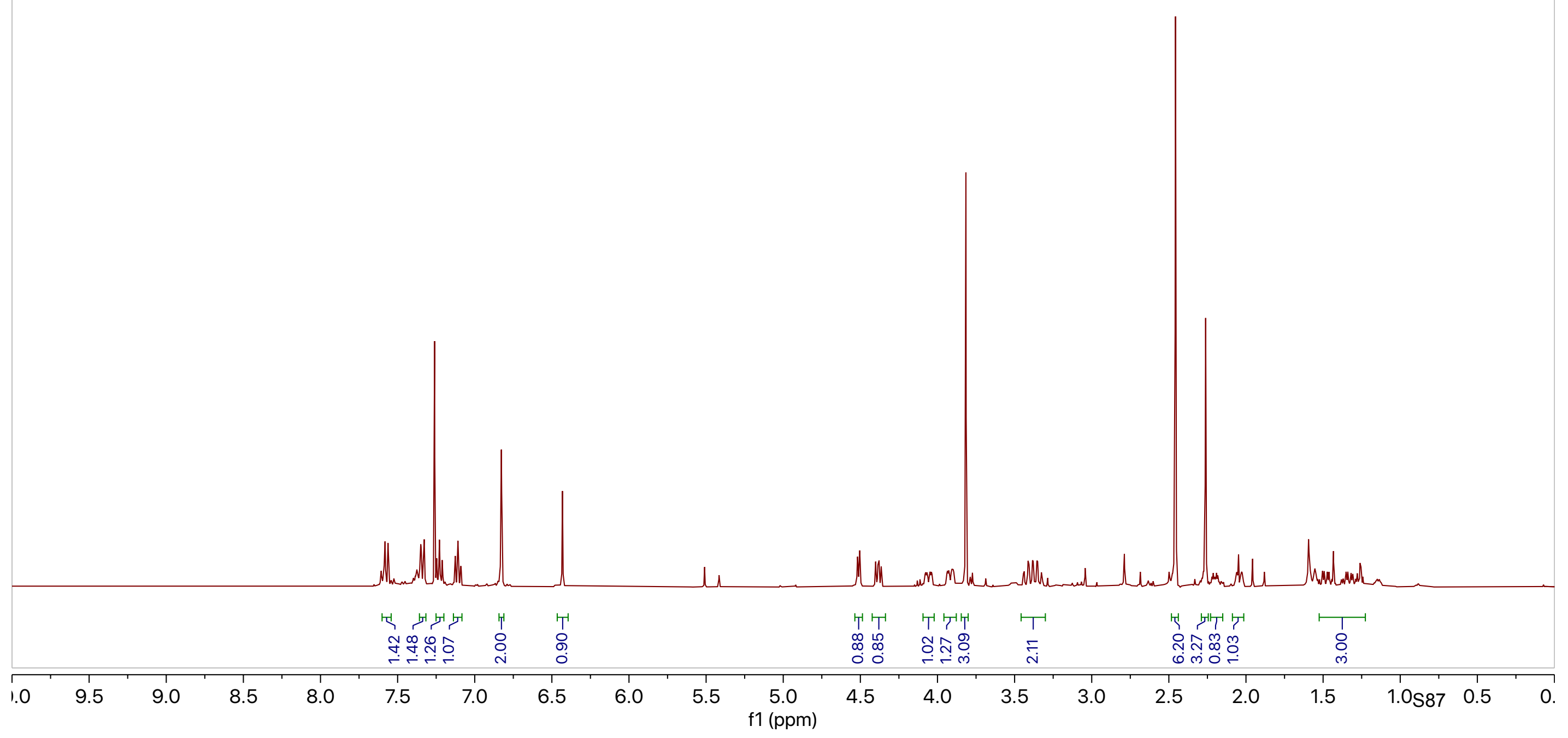




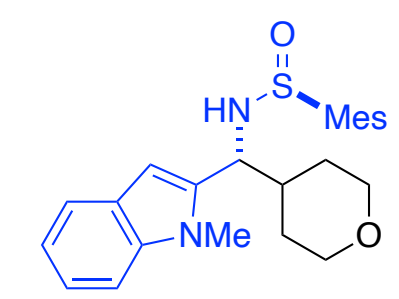

Compound 23

${ }^{13} \mathrm{C}$ NMR (101 MHz, $\mathrm{CDCl}_{3}$ )

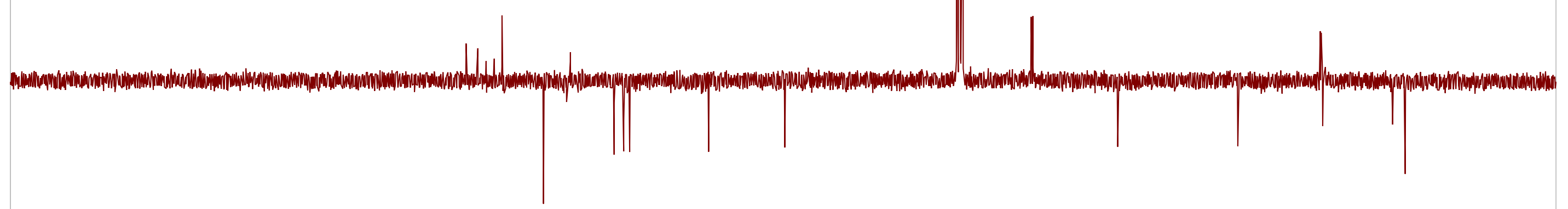

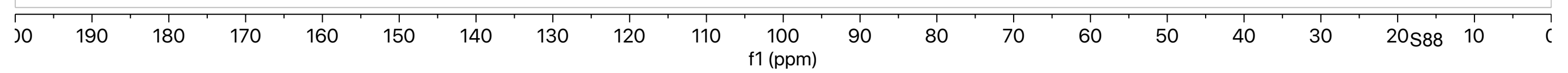




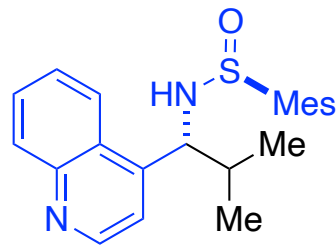

Compound 24 ${ }^{1} \mathrm{H}$ NMR $\left(400 \mathrm{MHz}, \mathrm{CDCl}_{3}\right)$

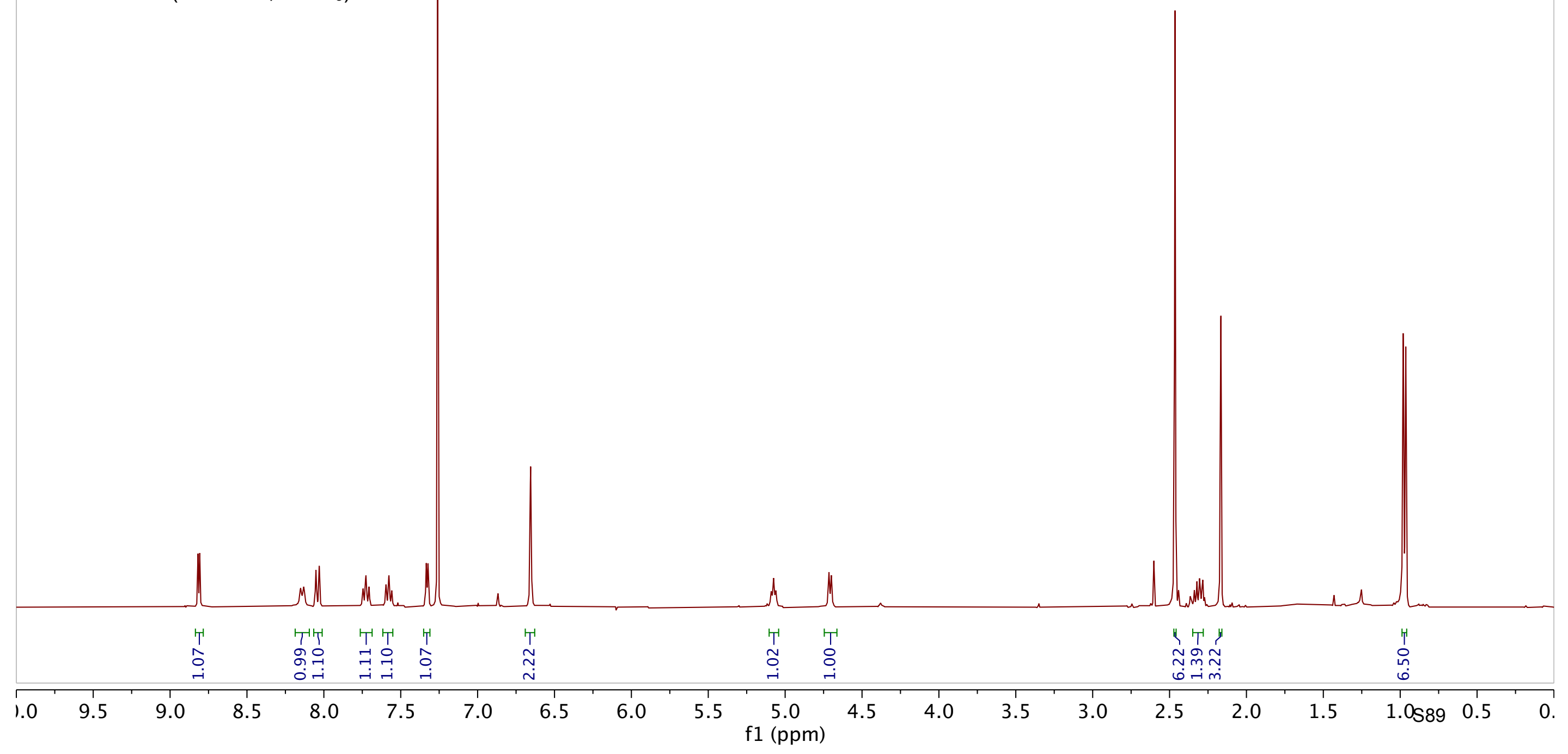




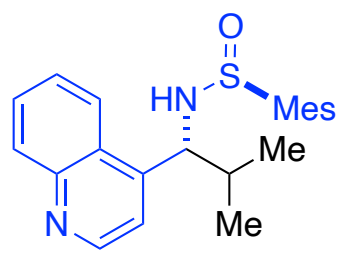

Compound 24

${ }^{13} \mathrm{C}$ NMR (101 MHz, $\mathrm{CDCl}_{3}$ )

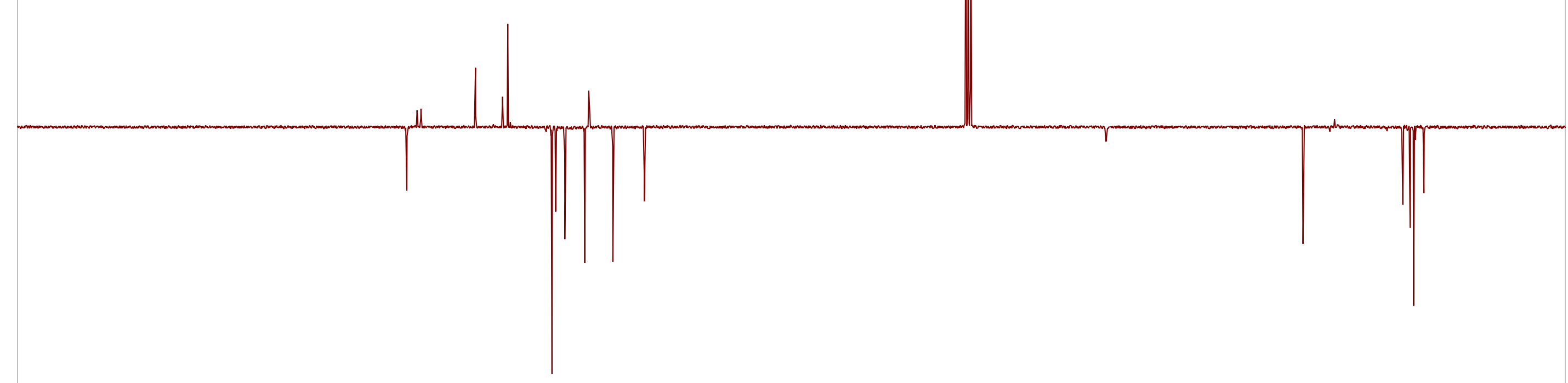

\begin{tabular}{|c|c|c|c|c|c|c|c|c|c|c|c|c|c|c|c|c|c|c|c|}
\hline 0 & 190 & 180 & 170 & 160 & 150 & 140 & 130 & 120 & 110 & $\begin{array}{c}100 \\
\mathrm{f} 1(\mathrm{ppm})\end{array}$ & 90 & 80 & 70 & 60 & 50 & 40 & 30 & $20 s 90 \quad 10$ & ( \\
\hline
\end{tabular}




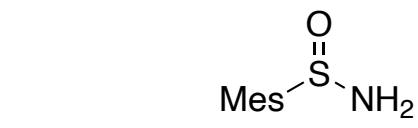

2,4,6-trimethylbenzenesulfinamide

${ }^{1} \mathrm{H}$ NMR $\left(600 \mathrm{MHz}, \mathrm{CDCl}_{3}\right)$

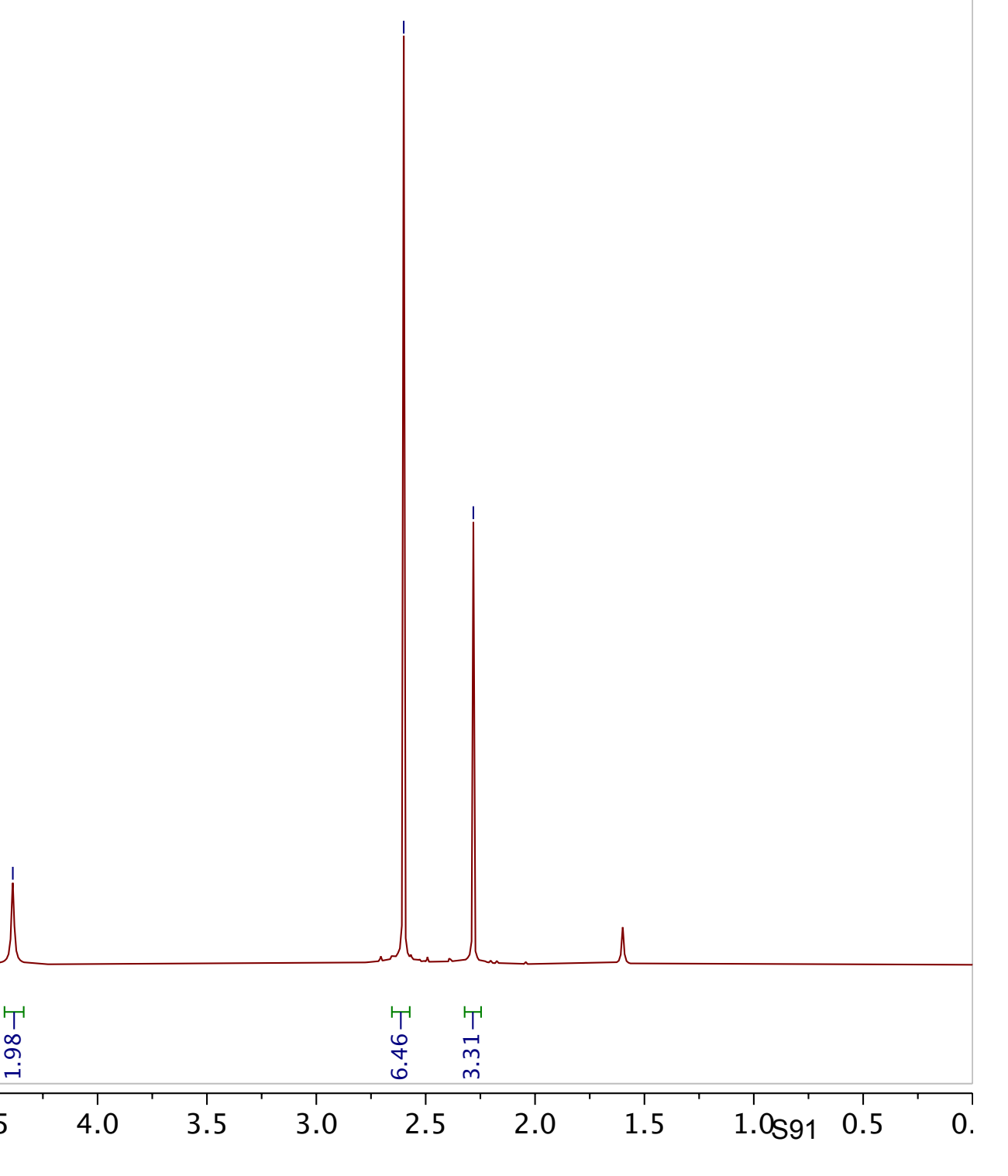




$$
\mathrm{Mes}^{-\mathrm{II}_{-}} \mathrm{NH}_{2}
$$

2,4,6-trimethylbenzenesulfinamide

$\left.{ }^{13} \mathrm{C} \mathrm{NMR} \mathrm{(151} \mathrm{MHz,} \mathrm{CDCl}_{3}\right)$

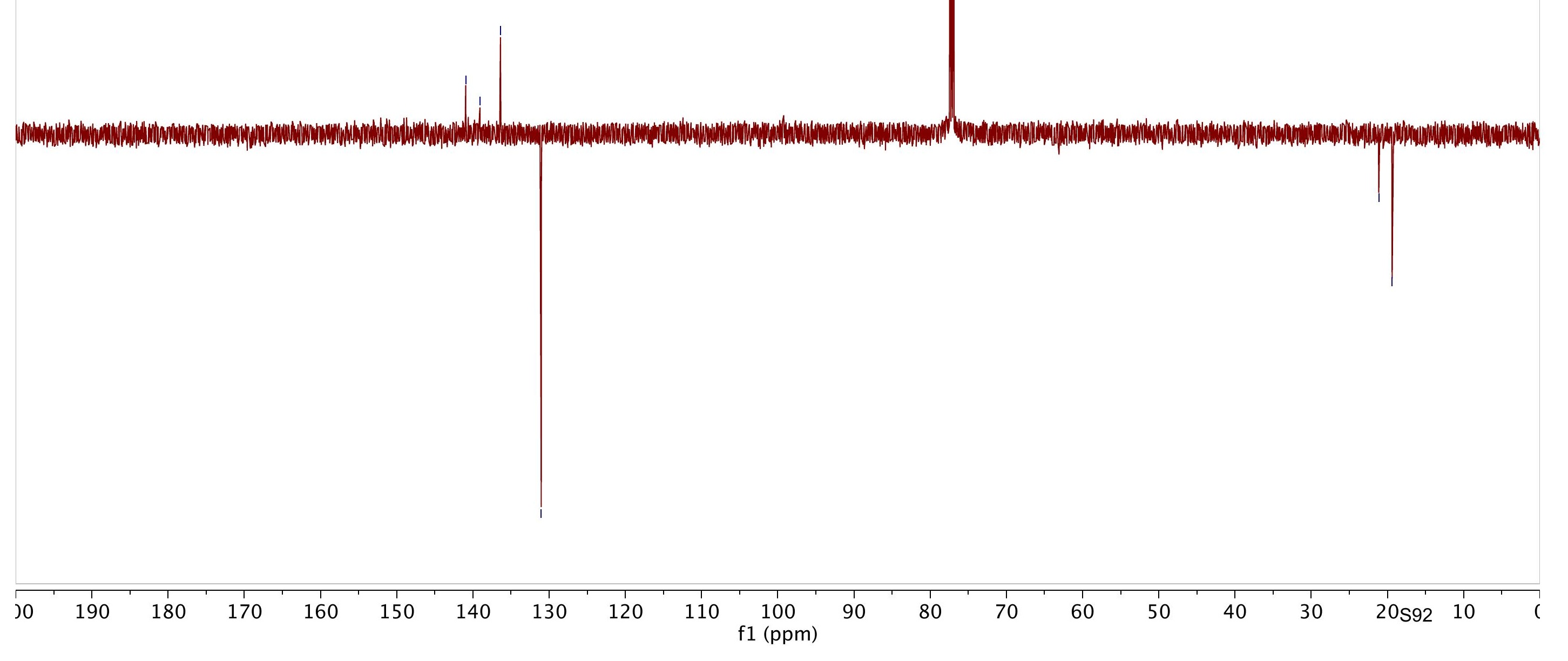




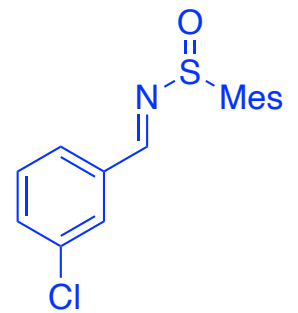

\section{Compound 2c}

${ }^{1} \mathrm{H}$ NMR $\left(400 \mathrm{MHz}, \mathrm{CDCl}_{3}\right)$ 


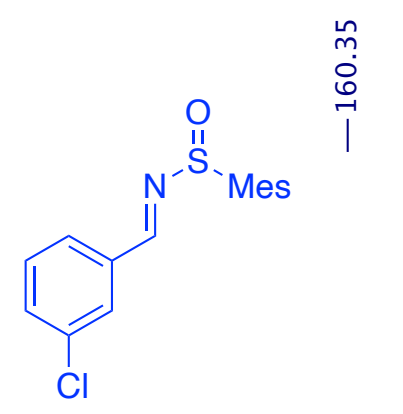

Compound 2c

${ }^{13} \mathrm{C}$ NMR (101 MHz, $\mathrm{CDCl}_{3}$ )

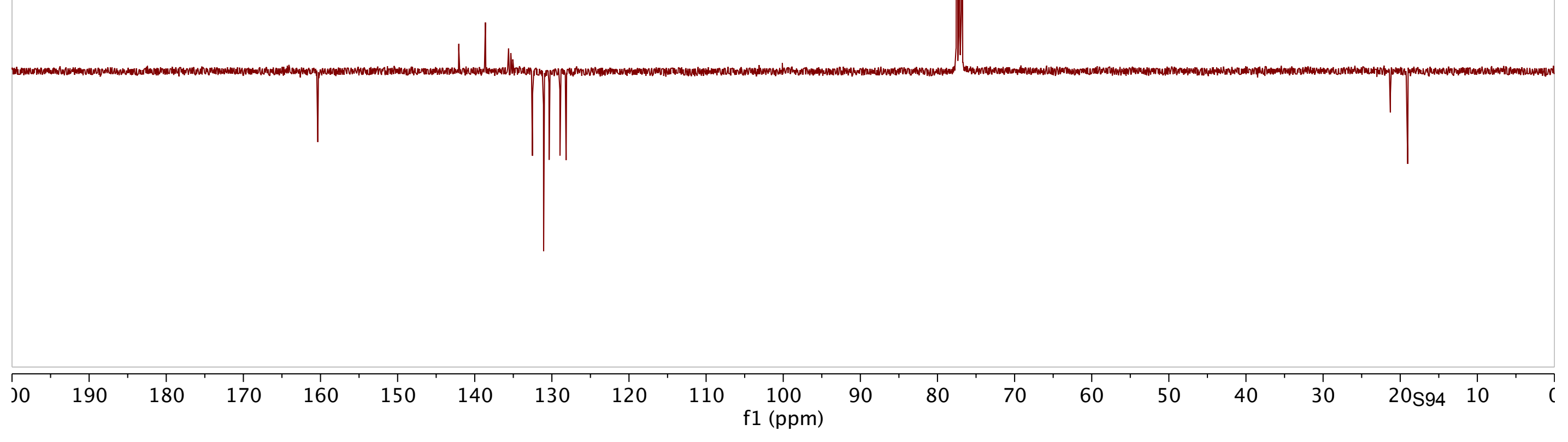




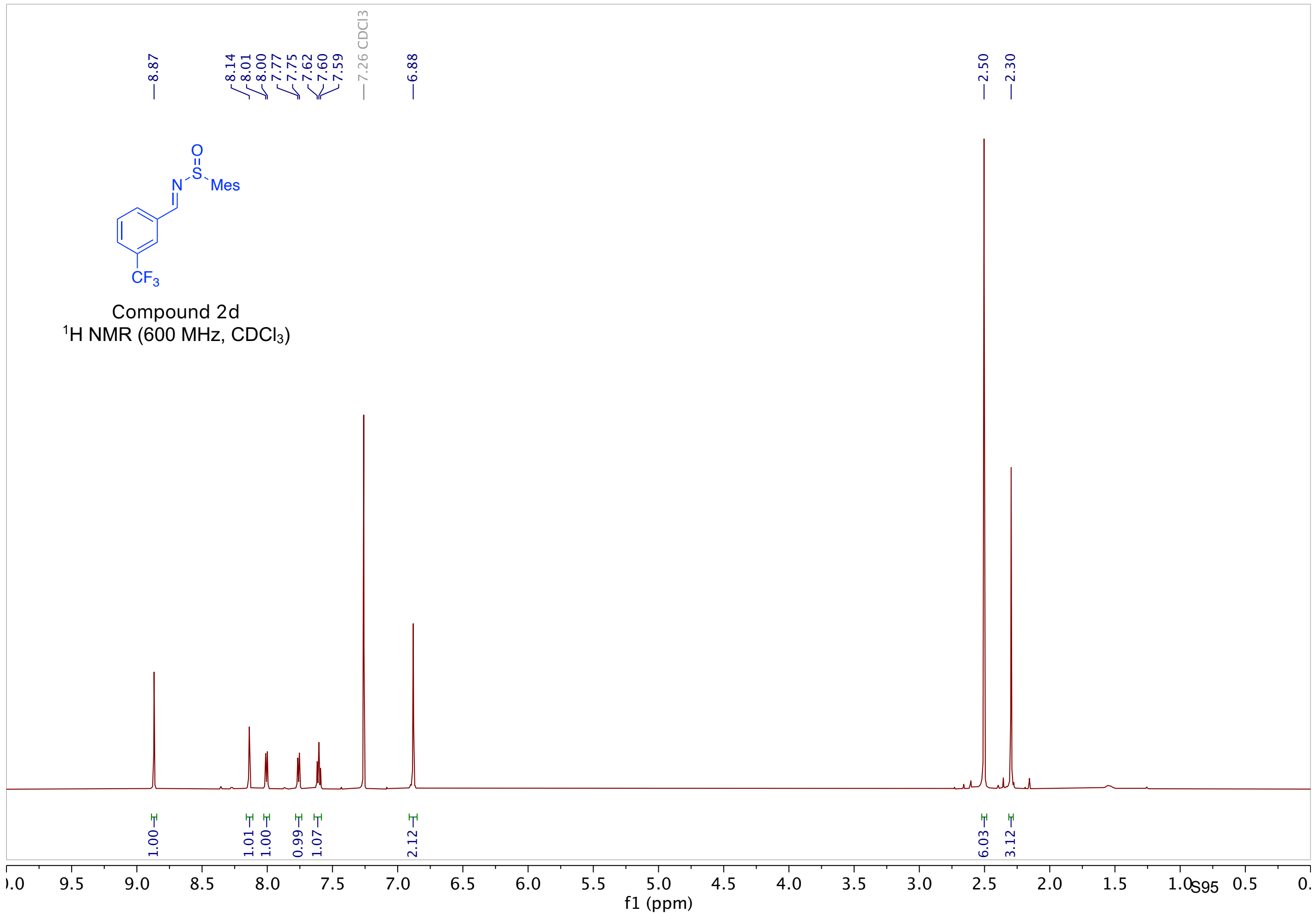




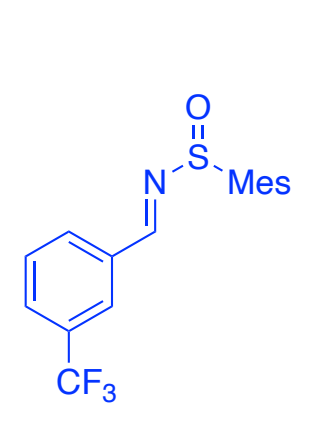

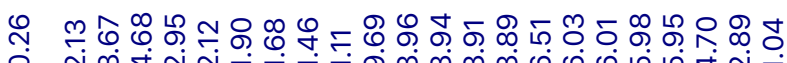

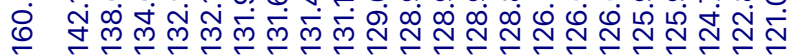

Compound 2d

${ }^{13} \mathrm{C}$ NMR $\left(151 \mathrm{MHz}, \mathrm{CDCl}_{3}\right)$
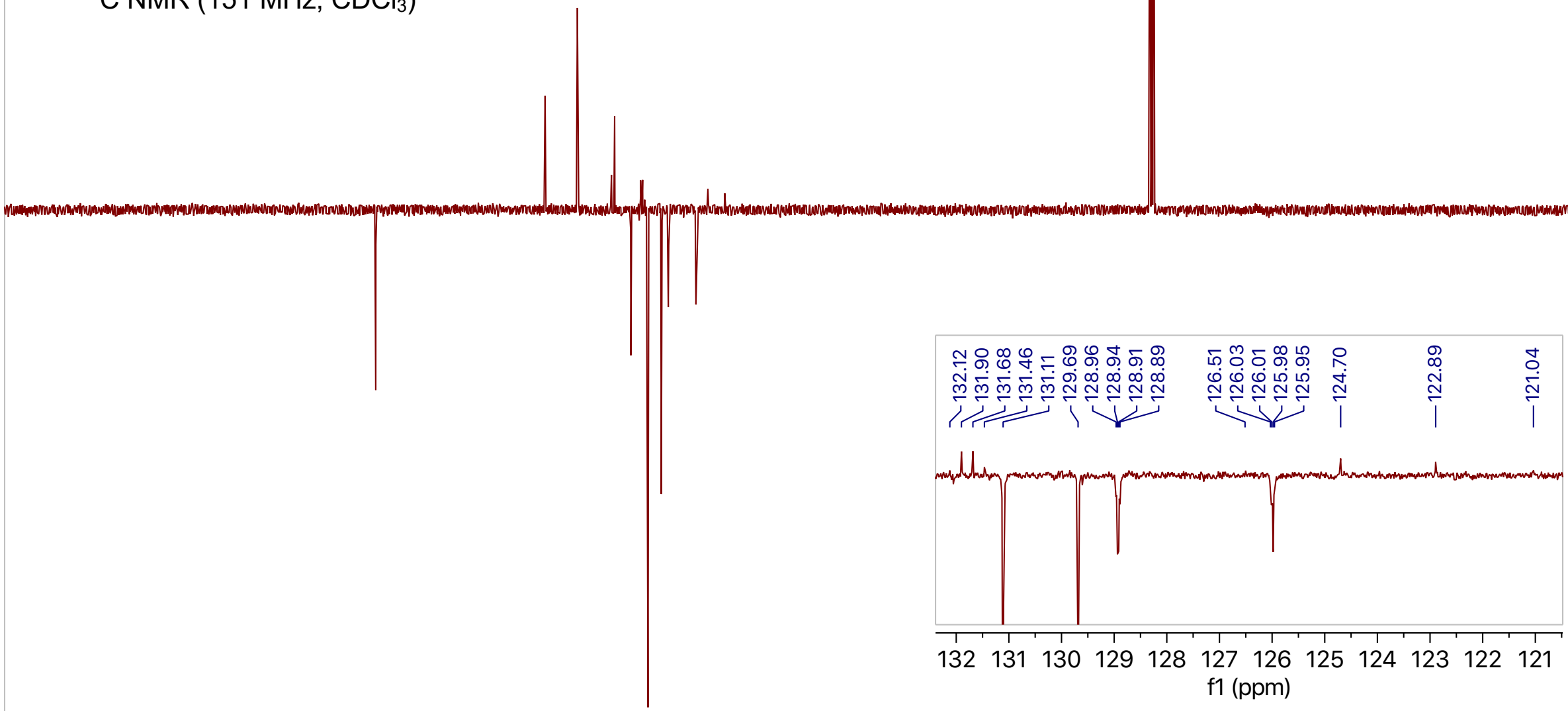


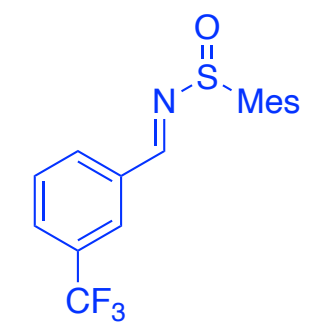

Compound $2 d$

${ }^{19} \mathrm{~F}$ NMR (565 MHz, $\mathrm{CDCl}_{3}$ ) 


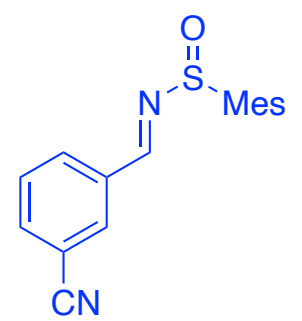

\section{Compound $2 \mathrm{e}$}

${ }^{1} \mathrm{H}$ NMR $\left(400 \mathrm{MHz}, \mathrm{CDCl}_{3}\right)$

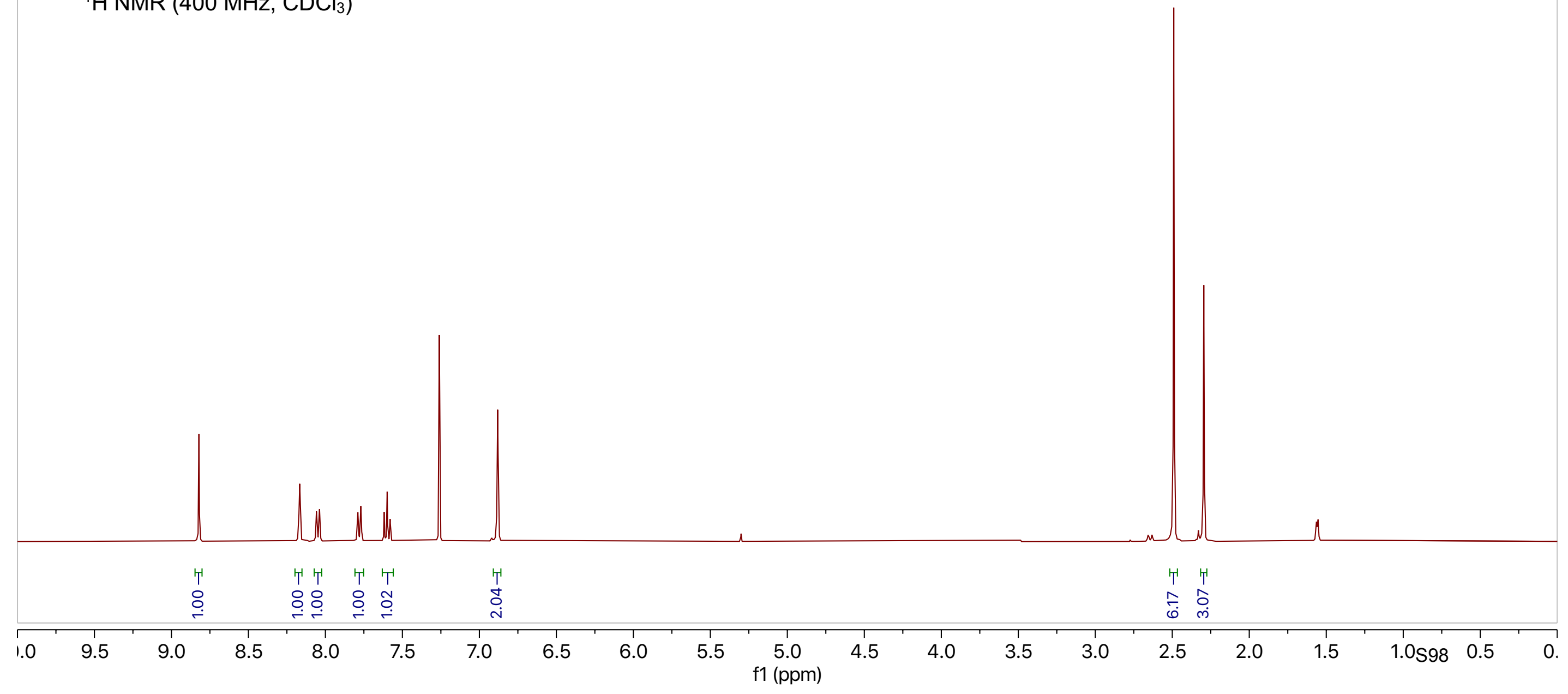




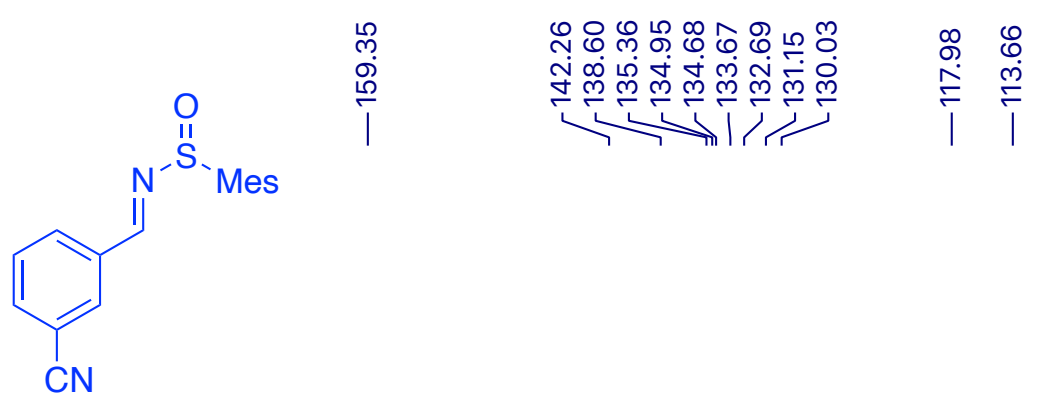

Compound $2 \mathrm{e}$

${ }^{13} \mathrm{C}$ NMR (101 MHz, $\mathrm{CDCl}_{3}$ )

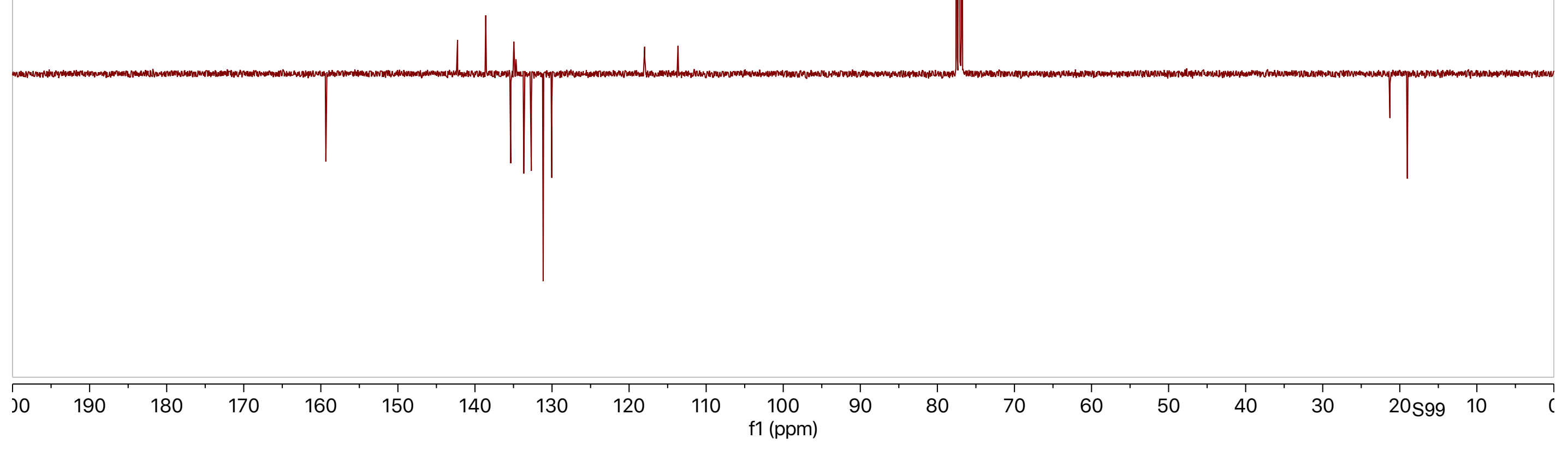




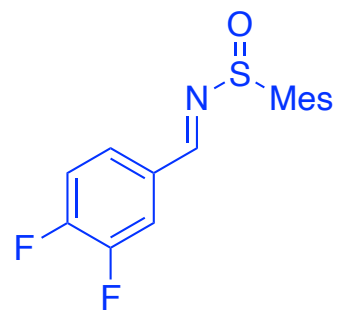

Compound $2 f$

${ }^{1} \mathrm{H} \mathrm{NMR}\left(600 \mathrm{MHz}, \mathrm{CDCl}_{3}\right)$

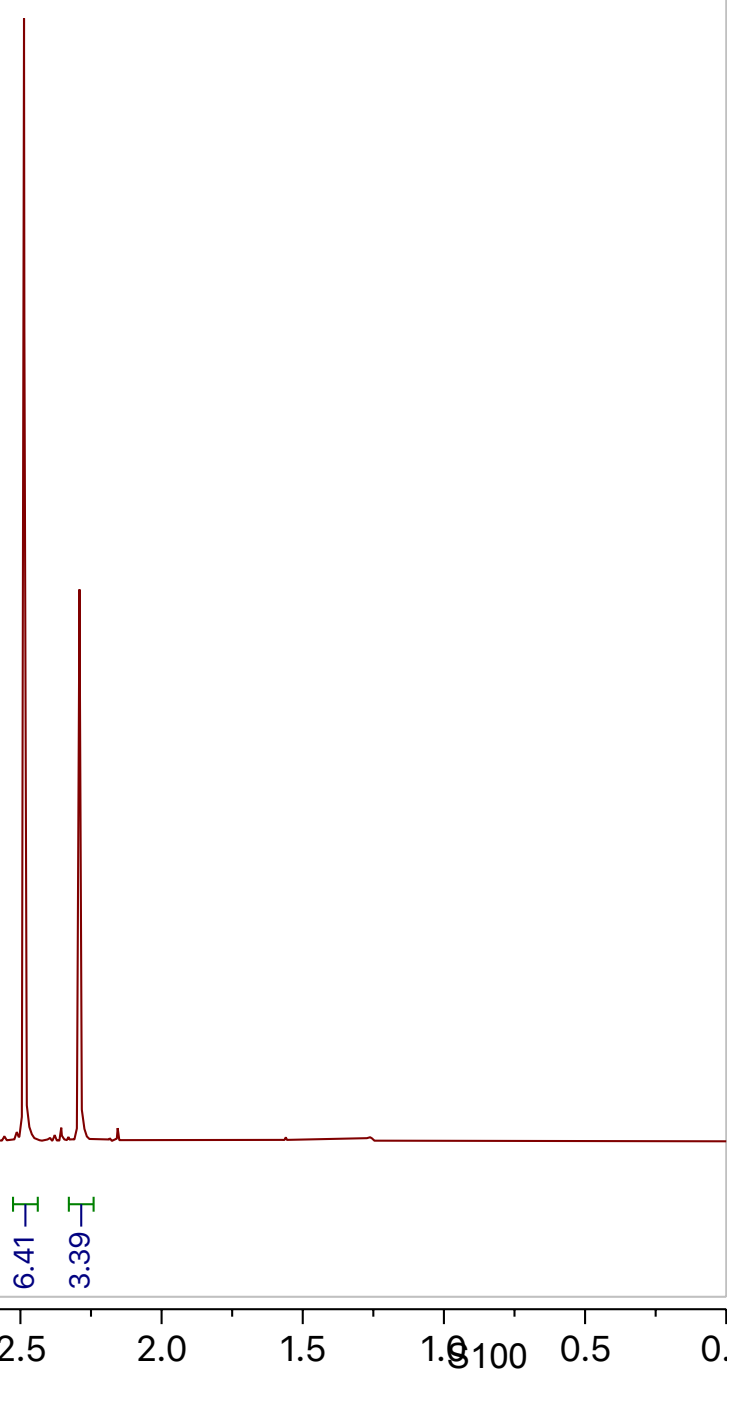




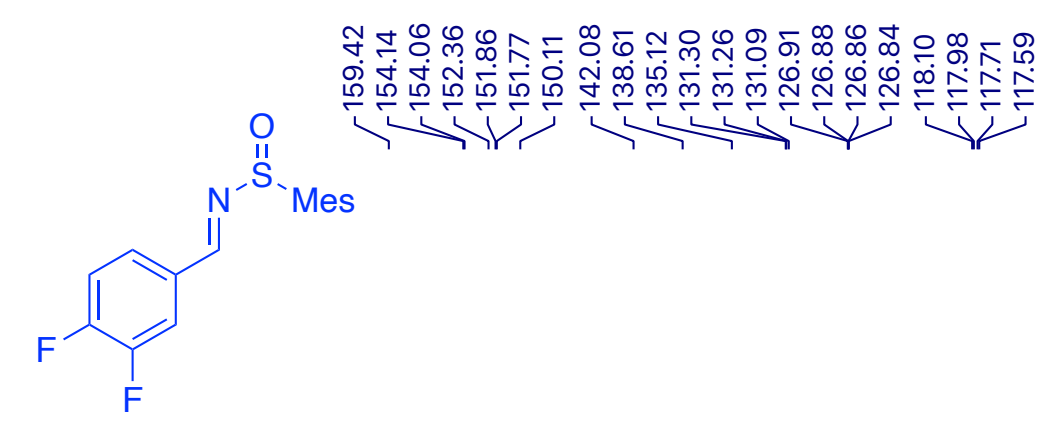

Compound $2 f$

${ }^{13} \mathrm{C}$ NMR (151 MHz, $\mathrm{CDCl}_{3}$ )
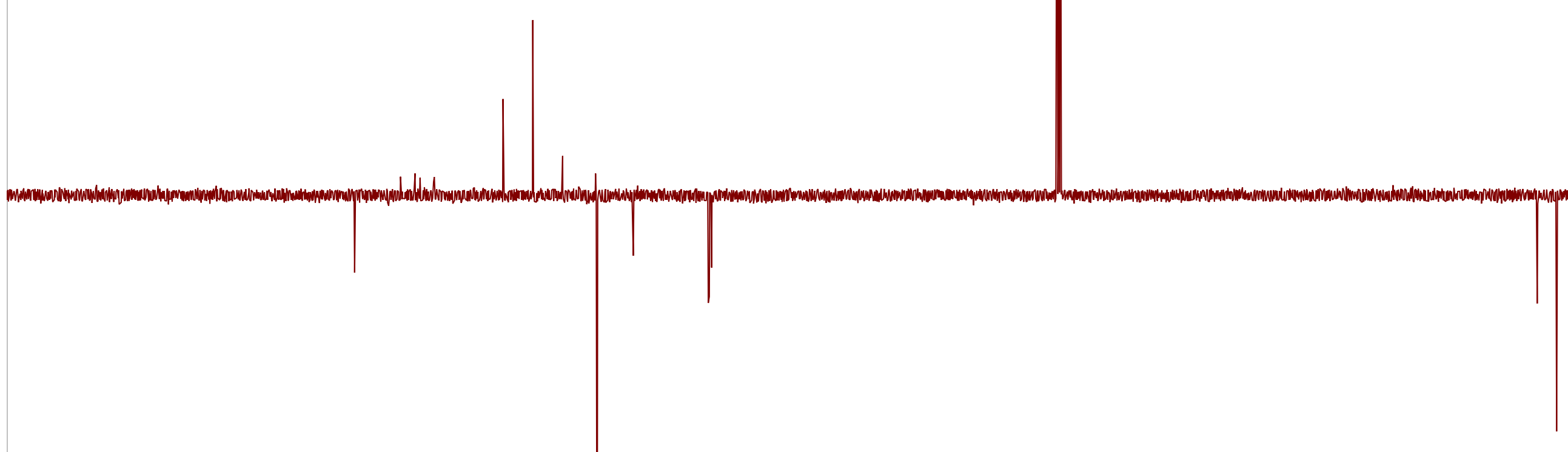


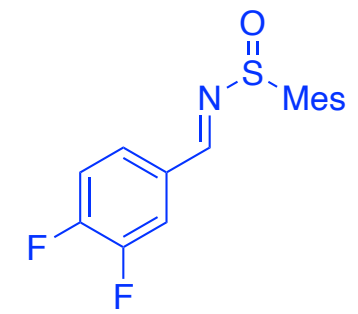

Compound $2 f$

${ }^{19} \mathrm{~F} \mathrm{NMR} \mathrm{(565} \mathrm{MHz,} \mathrm{CDCl}_{3}$ )

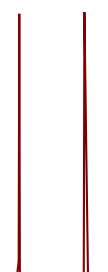




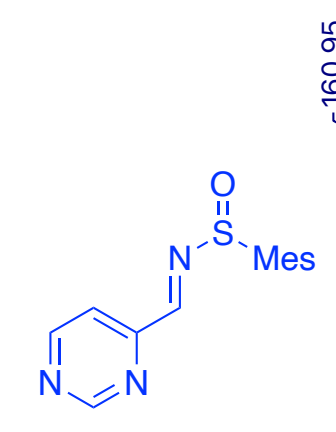

Compound $2 \mathrm{~g}$

${ }^{13} \mathrm{C}$ NMR (101 MHz, $\mathrm{CDCl}_{3}$ )

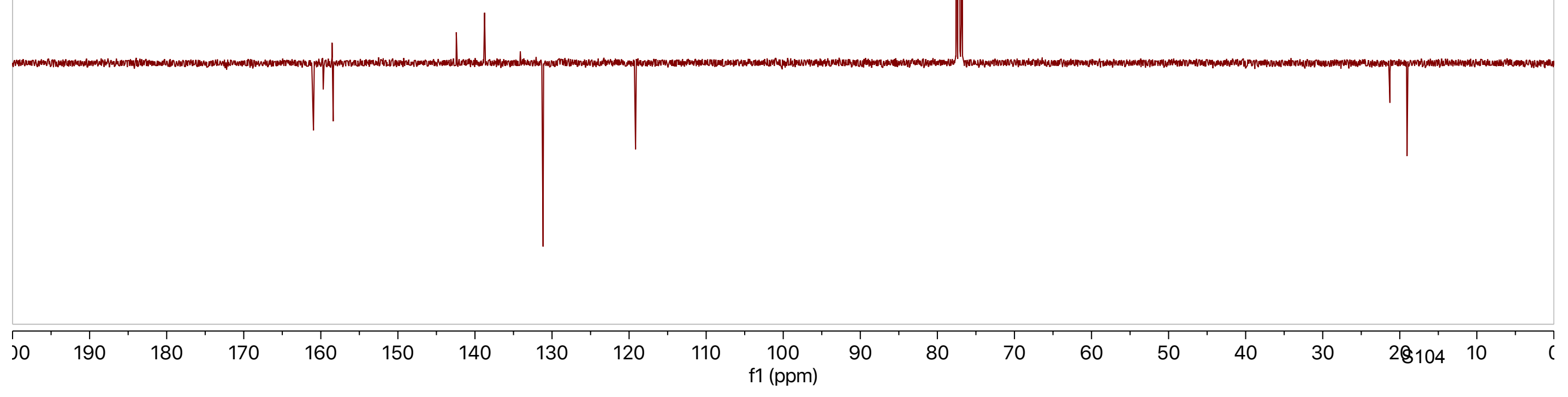




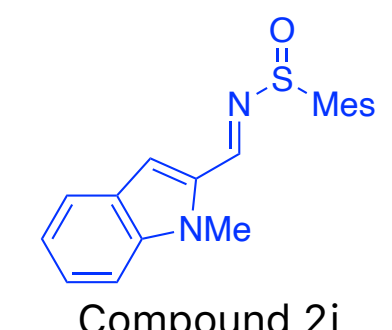

${ }^{1} \mathrm{H}$ NMR $\left(600 \mathrm{MHz}, \mathrm{CDCl}_{3}\right)$

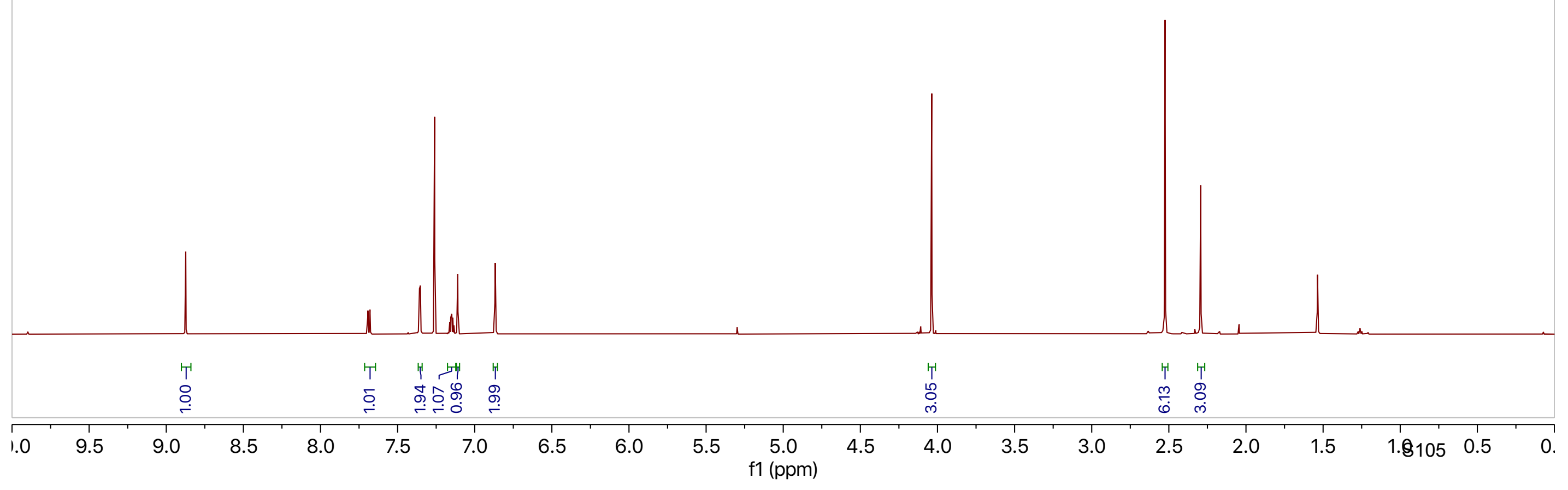




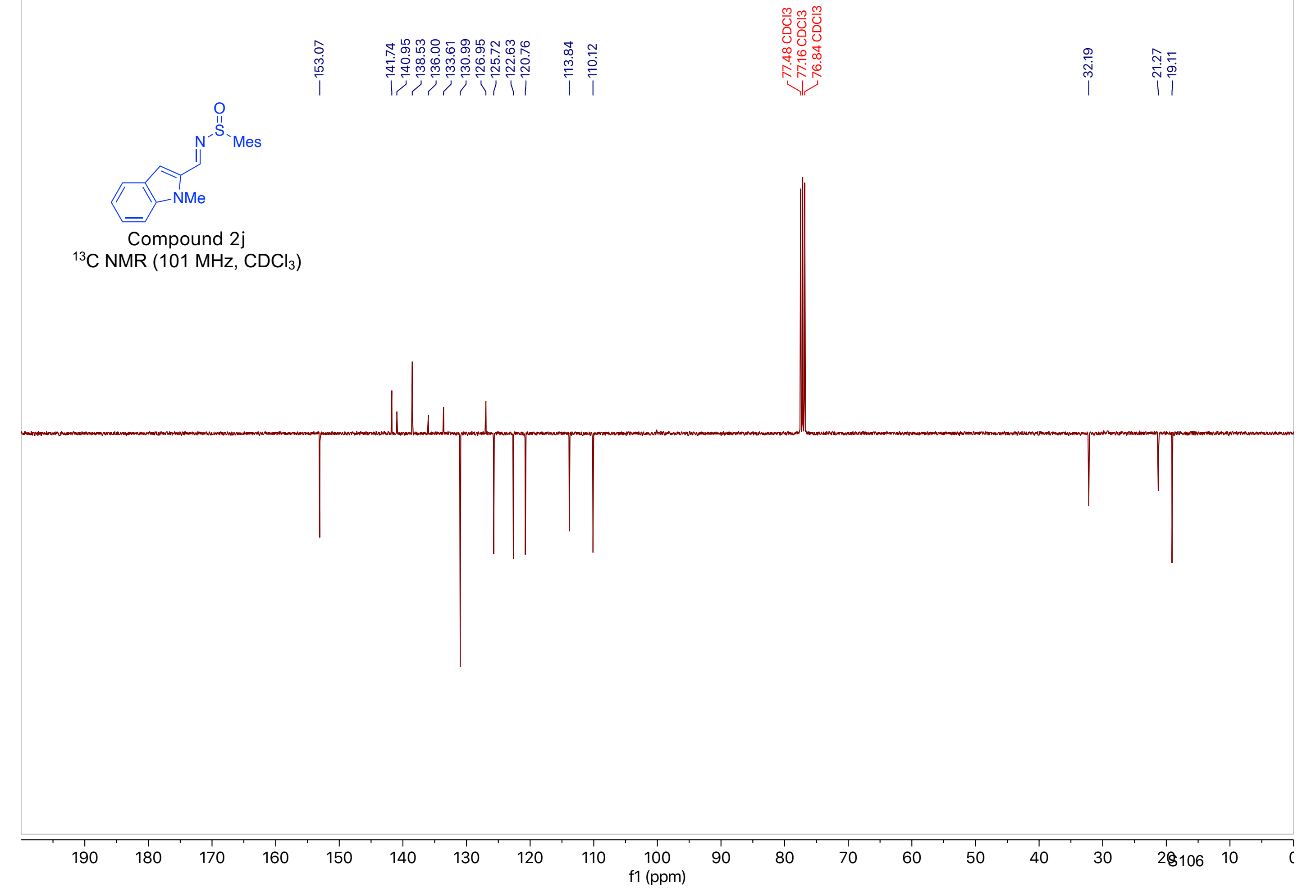




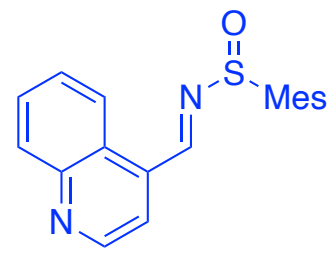

Compound $2 \mathrm{k}$

${ }^{1} \mathrm{H}$ NMR (400 MHz, $\mathrm{CDCl}_{3}$ )

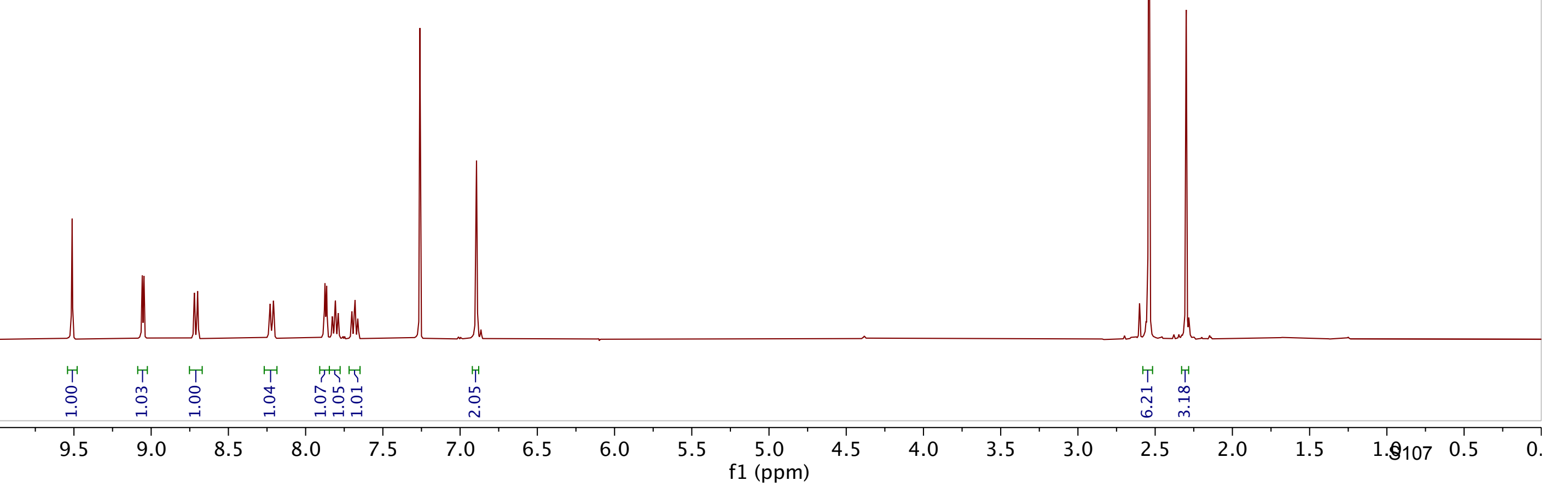




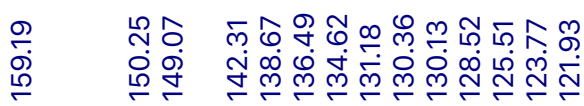

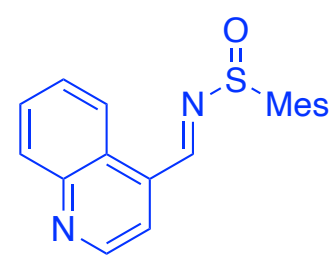

Compound 2k

${ }^{13} \mathrm{C}$ NMR (101 MHz, $\mathrm{CDCl}_{3}$ )

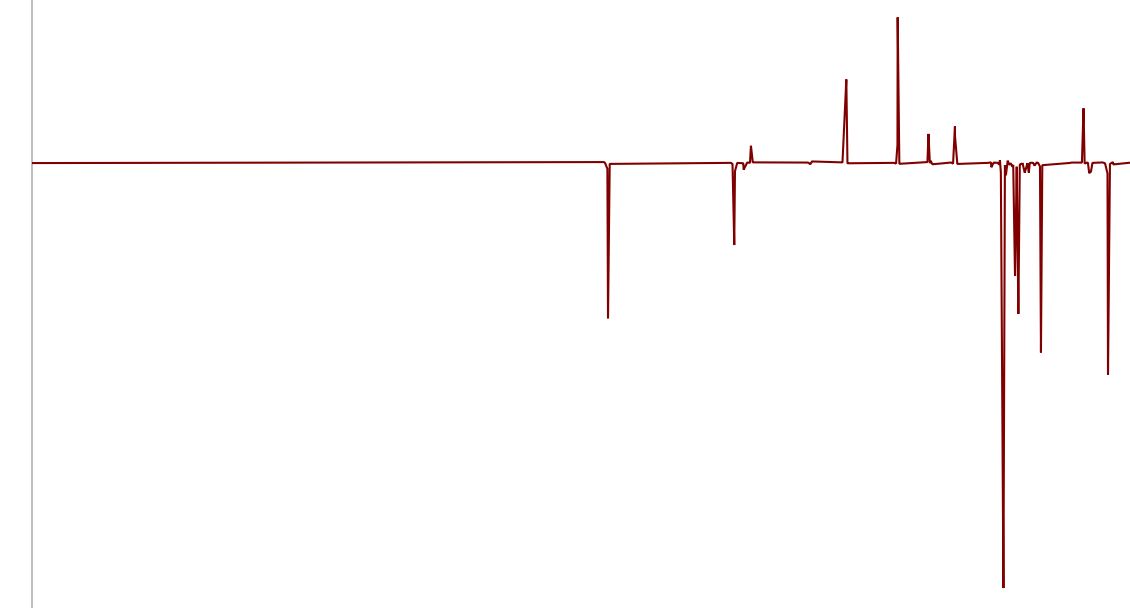




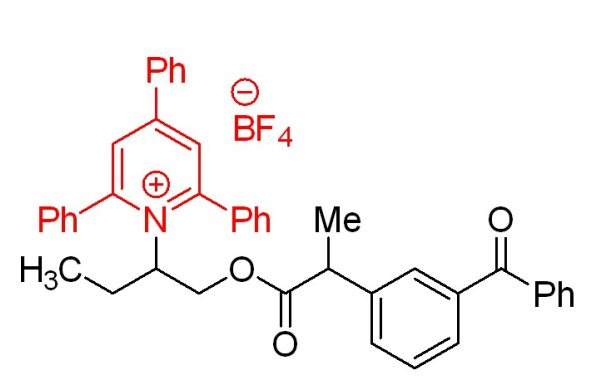

$\frac{\pi}{\pi+\pi}$
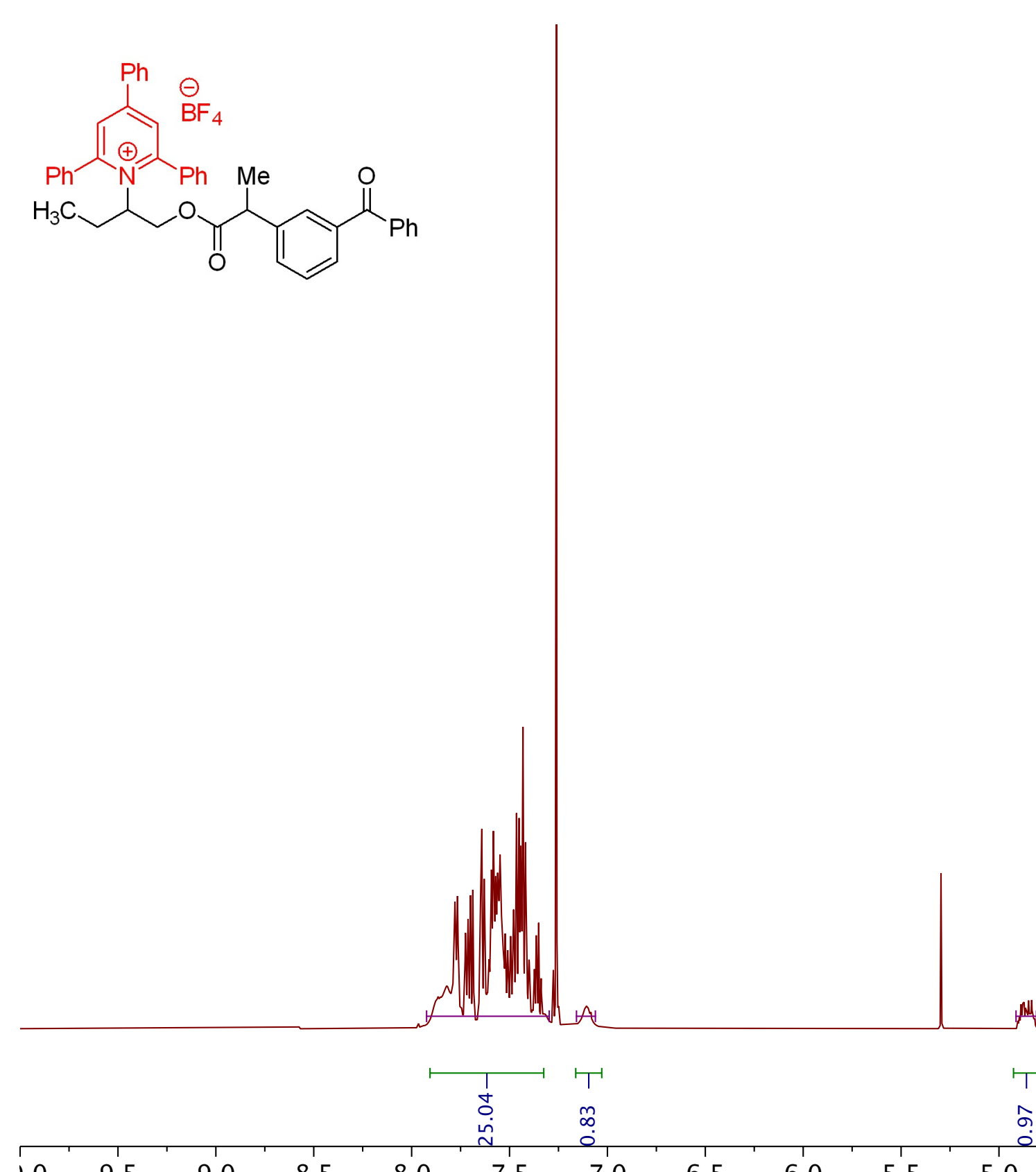

9.5

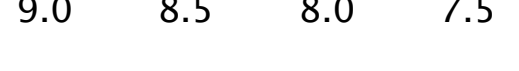
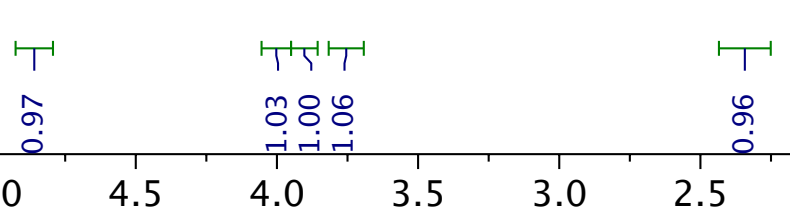

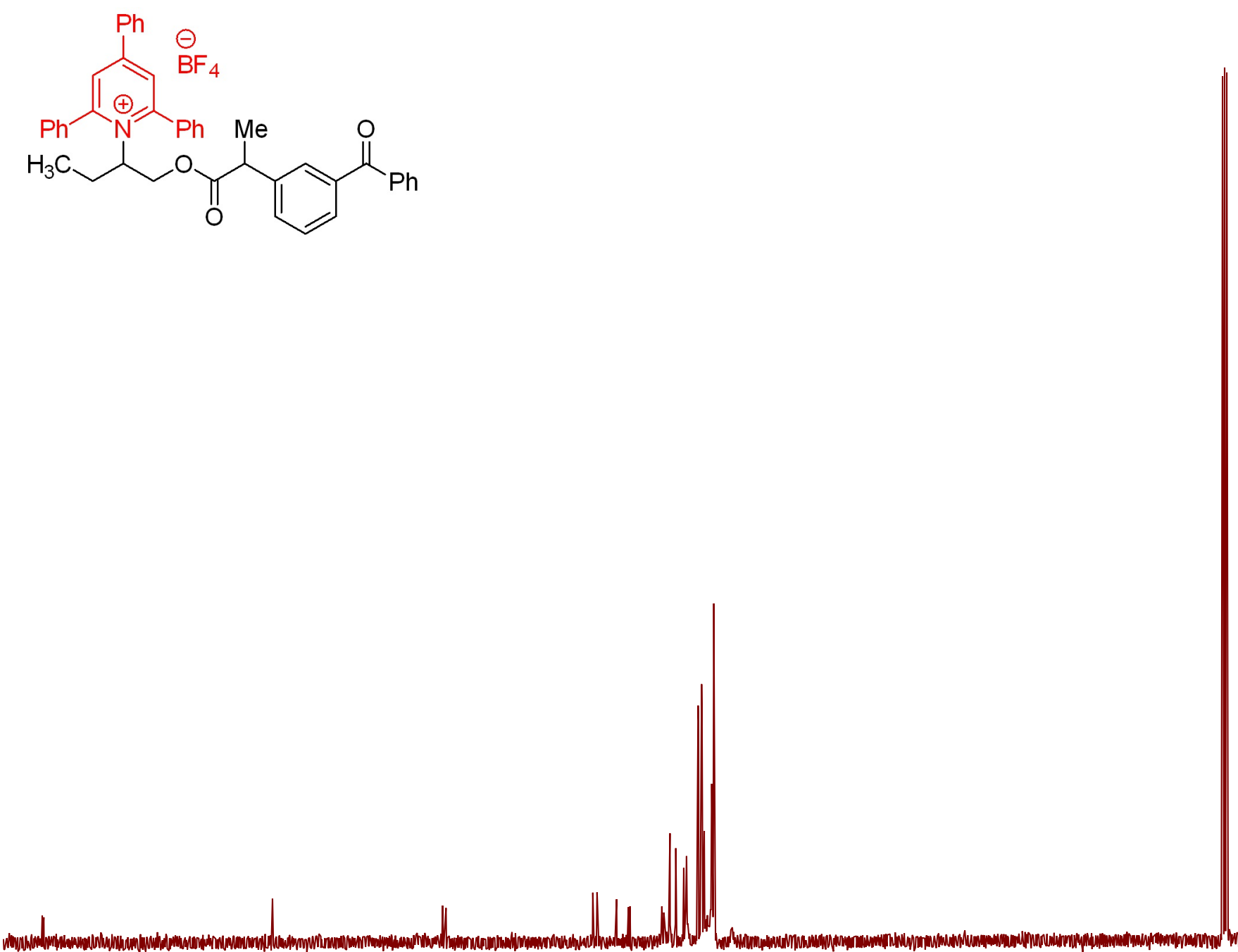

50

40




$$
\text { 要 }
$$

